NUREG/CR-6116

INEL-94/0039

Vol. 6

\title{
Systems Analysis Programs for Hands-on Integrated Reliability Evaluations (SAPHIRE) Version 5.0
}

\section{Graphical Evaluation Module (GEM) Reference Manual}

Manuscript Completed: September 1995

Date Published: October 1995

Prepared by

K. D. Russell, K. J. Kvarfordt, C. L. Hoffman,

R. E. Clark, M. B. Sattison

Idaho National Engineering Laboratory

Lockheed Idaho Technologies Company

Lockheed Idaho Technologies Company Idaho Falls, ID 83415

R. Robinson, NRC Project Manager

Prepared for

Division of Systems Technology

Office of Nuclear Regulatory Research

U.S. Nuclear Regulatory Commission

Washington, DC 20555-0001

NRC Job Code L1429 


\section{DISCLAIMER}

This report was prepared as an account of work sponsored by an agency of the United States Government. Neither the United States Government nor any agency thereof, nor any of their employees, make any warranty, express or implied, or assumes any legal liability or responsibility for the accuracy, completeness, or usefulness of any information, apparatus, product, or process disclosed, or represents that its use would not infringe privately owned rights. Reference herein to any specific commercial product, process, or service by trade name, trademark, manufacturer, or otherwise does not necessarily constitute or imply its endorsement, recommendation, or favoring by the United States Government or any agency thereof. The views and

opinions of authors expressed herein do not necessarily state or reflect those of the United States Government or any agency thereof. 


\section{DISCLAIMER}

Portions of this document may be illegible in electronic image products. Images are produced from the best available original document. 


\begin{abstract}
The Systems Analysis Programs for Hands-on Integrated Reliability Evaluations (SAPHIRE) refers to a set of several microcomputer programs that were developed to create and analyze probabilistic risk assessments (PRAs), primarily for nuclear power plants. The Graphical Evaluation Module (GEM) is a special application tool designed for evaluation of operational occurrences using the Accident Sequence Precursor (ASP) program methods. GEM provides the capability for an analyst to quickly and easily perform conditional core damage probability (CCDP) calculations. The analyst can then use the CCDP calculations to determine if the occurrence of an initiating event or a condition adversely impacts safety. It uses models and data developed in the SAPHIRE specially for the ASP program. GEM requires more data than that normally provided in SAPHIRE and will not perform properly with other models or data bases.
\end{abstract}

This is the first release of GEM and the developers of GEM welcome user comments and feedback that will generate ideas for improvements to future versions. GEM is designated as version 5.0 to track GEM codes along with the other SAPHIRE codes as the GEM relies on the same, shared database structure.

Fin No. L1429 - SAPHIRE Maintenance and User Support 


\section{Documents in NUREG/CR-6116 Report, Systems Analysis Programs for Hands-on Integrated Reliability Evaluations (SAPHIRE) \\ Version $\mathbf{5 . 0}$}

Volume 1 - Technical Reference Manual

Volume 2 - Integrated Reliability and Risk Analysis System (IRRAS) Reference Manual

Volume 3 - Integrated Reliability and Risk Analysis System (IRRAS) Tutorial Manual

Volume 4 - Systems Analysis and Risk Assessment (SARA) System Reference Manual

Volume 5 - Systems Analysis and Risk Assessment (SARA) System Tutorial Manual

Volume 6 - Graphical Evaluation Module (GEM) Reference Manual

Volume 7 - Fault Tree, Event Tree, and Piping \& Instrumentation Diagram (FEP) Editors Reference Manual

Volume 8 - Models and Results Database (MAR-D) Reference Manual

Volume $9-$ Validation and Verification (V\&V) Manual

Volume 10 - Data Loading Manual 


\section{CONTENTS}

ABSTRACT $\ldots \ldots \ldots \ldots \ldots \ldots \ldots \ldots \ldots \ldots \ldots \ldots \ldots \ldots \ldots \ldots \ldots \ldots$ ii

FIGURES $\ldots \ldots \ldots \ldots \ldots \ldots \ldots \ldots \ldots \ldots \ldots \ldots \ldots \ldots \ldots \ldots \ldots \ldots$

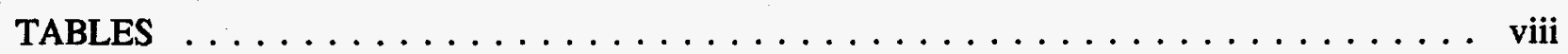

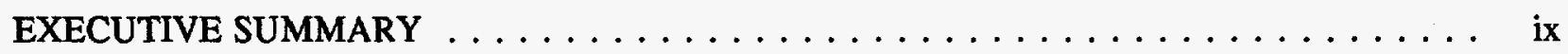

FOREWORD $\ldots \ldots \ldots \ldots \ldots \ldots \ldots \ldots \ldots \ldots \ldots \ldots \ldots \ldots \ldots \ldots \ldots \ldots$

ACRONYMS $\ldots \ldots \ldots \ldots \ldots \ldots \ldots \ldots \ldots \ldots \ldots \ldots \ldots \ldots \ldots \ldots \ldots \ldots \ldots$ xiii

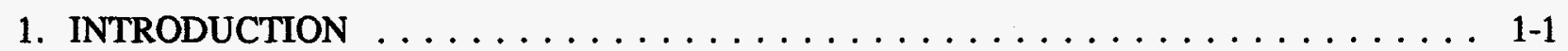

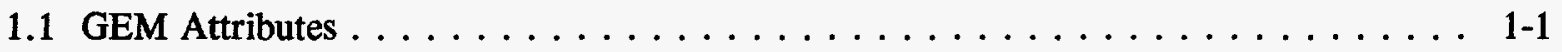

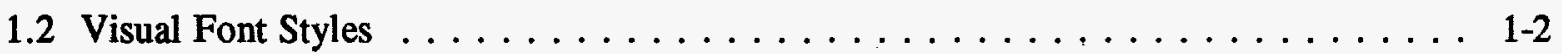

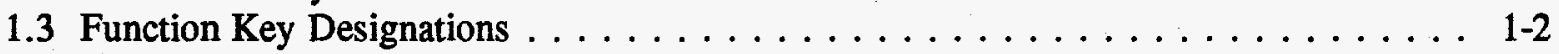

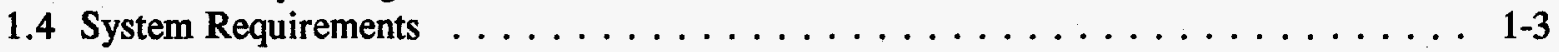

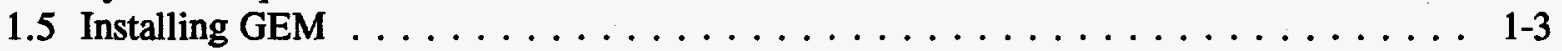

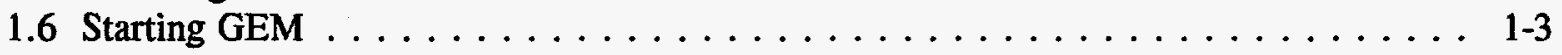

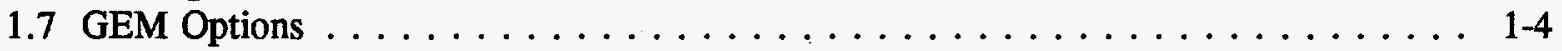

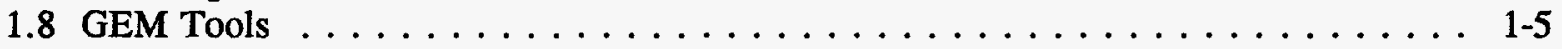

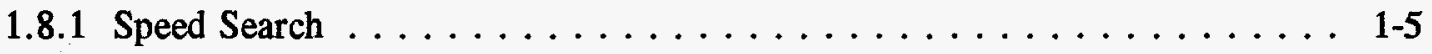

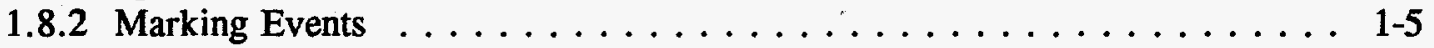

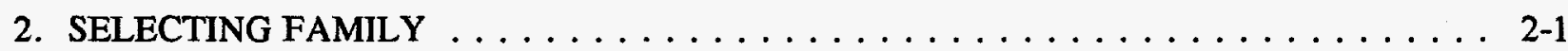

3. INITIATING EVENT ASSESSMENT . . . . . . . . . . . . . . . . . 3-1

3.1 Performing an Initiating Event Assessment $\ldots \ldots \ldots \ldots \ldots \ldots \ldots \ldots$ 3-1

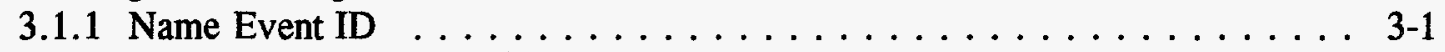

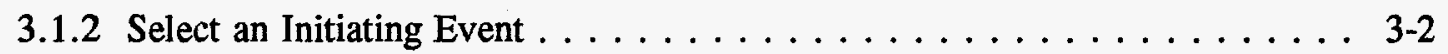

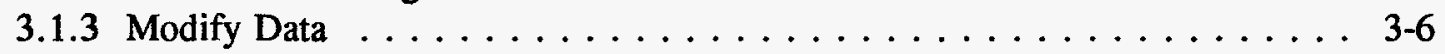

3.1.4 Event Assessment . . . . . . . . . . . . . . . . . 3-9

3.2 Retrieving an Initiating Event Assessment $\ldots \ldots \ldots \ldots \ldots \ldots \ldots \ldots . \ldots \ldots$ 3-26

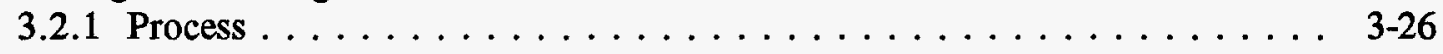

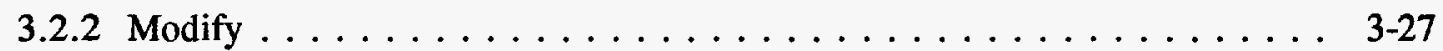

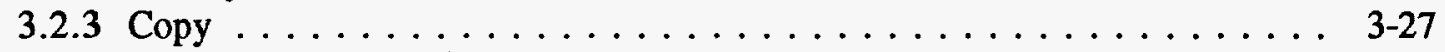

3.2.4 Delete $\ldots \ldots \ldots \ldots \ldots \ldots \ldots \ldots \ldots \ldots \ldots \ldots \ldots \ldots$ 3-27

3.2.5 Rename . . . . . . . 3-29

3.3 Event Probability Changes Screen $\ldots \ldots \ldots \ldots \ldots \ldots \ldots \ldots \ldots \ldots$ 3-29

3.3.1 Event Probability Changes Screen Online Help . . . . . . . . . 3-31

4. CONDITION ASSESSMENT $\ldots \ldots \ldots \ldots \ldots \ldots \ldots \ldots \ldots \ldots \ldots$ 4-1

4.1 Performing a Condition Assessment $\ldots \ldots \ldots \ldots \ldots \ldots \ldots \ldots \ldots$ 4-1

4.1 .1 Name Event ID . . . . . . . . . . . . . . . 4-1

4.1.2 Designate the Event Duration $\ldots \ldots \ldots \ldots \ldots \ldots \ldots \ldots \ldots .4 .2$ 
4.1.3 Modify Data $\ldots \ldots \ldots \ldots \ldots \ldots \ldots \ldots \ldots \ldots \ldots \ldots \ldots \ldots \ldots$

4.1 .4 Condition Assessment . . . . . . . . . . . . . . . . . .

4.2 Retrieving a Condition Assessment $\ldots \ldots \ldots \ldots \ldots \ldots \ldots \ldots \ldots \ldots$ 4-10

4.3 Event Probability Changes Screen $\ldots \ldots \ldots \ldots \ldots \ldots \ldots \ldots \ldots \ldots$ 4-10

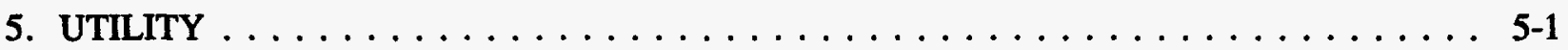

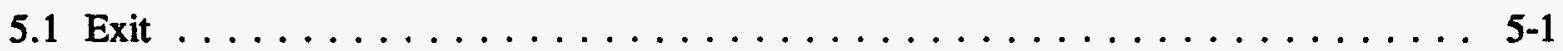

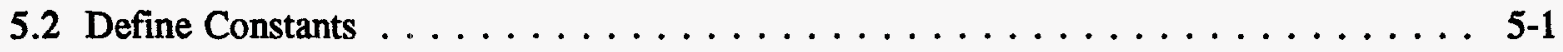

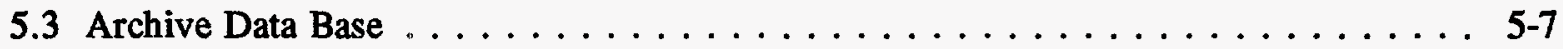

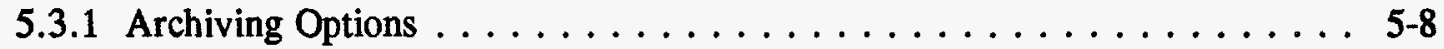

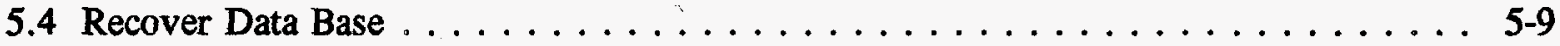

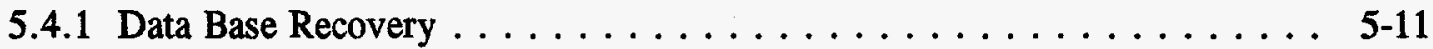

5.4.2 Cross Reference Recovery . . . . . . . . . . . . . 5-11

5.4.3 System Logic Events $\ldots \ldots \ldots \ldots \ldots \ldots \ldots \ldots \ldots \ldots$ 5-11

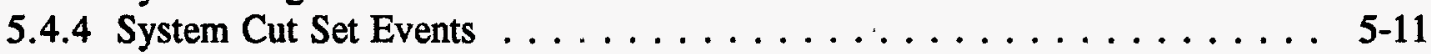

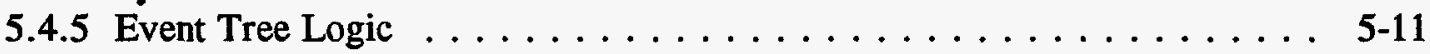

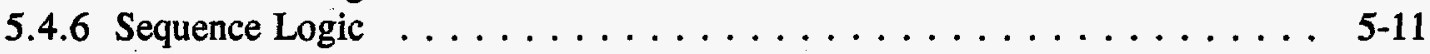

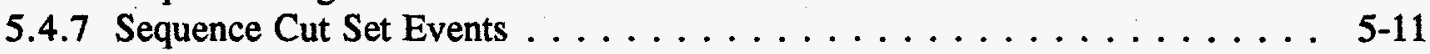

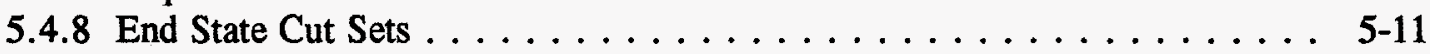

5.4 .9 Histogram Event $\ldots \ldots \ldots \ldots \ldots \ldots \ldots \ldots \ldots \ldots \ldots \ldots .5-11$

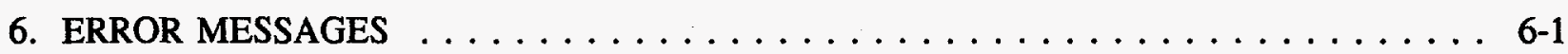

Appendix A Installation Procedure $\ldots \ldots \ldots \ldots \ldots \ldots \ldots \ldots \ldots \ldots \ldots \ldots \ldots \ldots \ldots \ldots$

Appendix B GEM Technical Reference $\ldots \ldots \ldots \ldots \ldots \ldots \ldots \ldots \ldots \ldots$ B-1

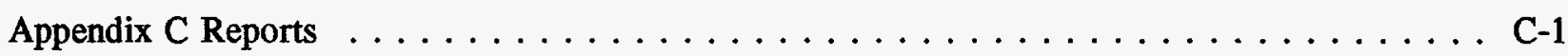




\section{FIGURES}

Figure 1. GEM main menu. . . . . . . . . . . . . . . . . . . . . 1-4

Figure 2. Select Family screen. . . . . . . . . . . . . . . . . . . 2-1

Figure 3. Name Event ID screen. . . . . . . . . . . . . . . . . . . . . 3-2

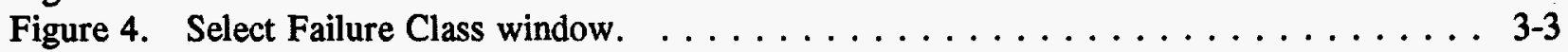

Figure 5. Recovery Event(s) screen for initiating events other than LOOP initiating events. . . . 3-4

Figure 6. Example of a select failure class screen. . . . . . . . . . . . . . . . 3-4

Figure 7. LOOP Nonrecovery Values screen $\ldots \ldots \ldots \ldots \ldots \ldots \ldots \ldots \ldots$

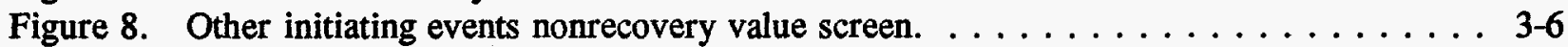

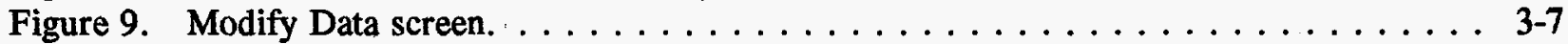

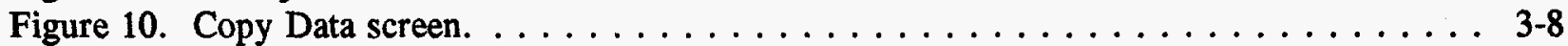

Figure 11. Event Assessment screen. . . . . . . . . . . . . . . . . . . . . 3-9

Figure 12. Reports main menu. . . . . . . . . . . . . . . . . . . . . 3-10

Figure 13. Sequence and Cut Set Contributions and Basic Event Parameters window. . . . . . 3-11

Figure 14. Cut Set screen. . . . . . . . . . . . . . . . . . . . . 3-16

Figure 15. Basic Events screen. . . . . . . . . . . . . . . . . . 3-17

Figure 16. Sequence Logic screen. . . . . . . . . . . . . . . . . . 3-18

Figure 17. Importance screen. . . . . . . . . . . . . . . . . . . 3-19

Figure 18. Partition screen. . . . . . . . . . . . . . . . . . . . 3-20

Figure 19. Example of the Importance screen using the Include option. . . . . . . . . . . . 3-21

Figure 20. Example of the Importance screen using the Exclude option. . . . . . . . . . . 3-22

Figure 21. Example of the Importance screen using the Complement option. . . . . . . . . 3-23

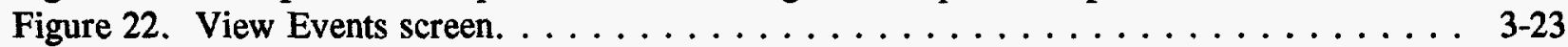

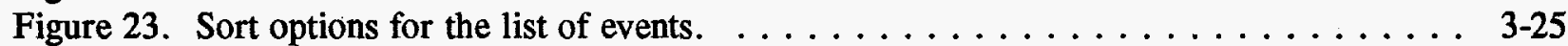

Figure 24. Select Sequence Sort window. . . . . . . . . . . . . . . . . . 3-25

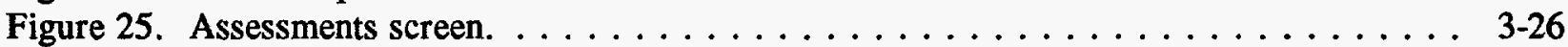

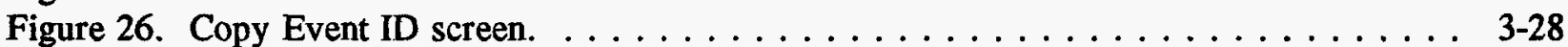

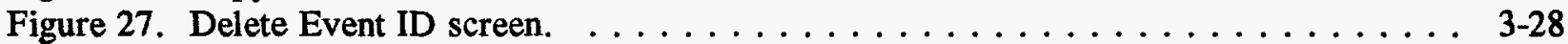

Figure 28. Rename Event ID screen. . . . . . . . . . . . . . . . . . . . . . . . . . 3-29

Figure 29. Example of the Event Probability Changes screen for a single event case. . . . . 3-30

Figure 30. Example of the Event Probability Changes screen for the group of events case. . . . 3-30

Figure 31. Failure Data Calculations Types online help screen. . . . . . . . . . . 3-32

Figure 32. Uncertainty Distribution Types online help screen. . . . . . . . . . . . 3-32

Figure 33. Process Flag online help screen. . . . . . . . . . . . . . . . 3-33

Figure 34. Name Event ID screen. . . . . . . . . . . . . . . . . . . . . 4-2

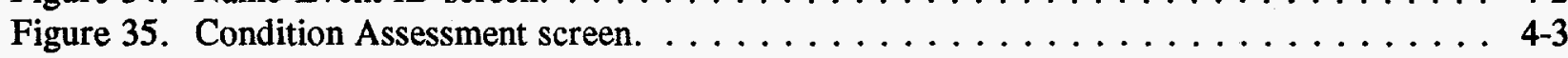

Figure 36. Condition Assessment screen. . . . . . . . . . . . . . . . . . 4-4

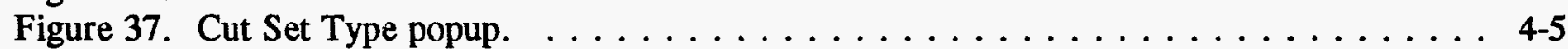

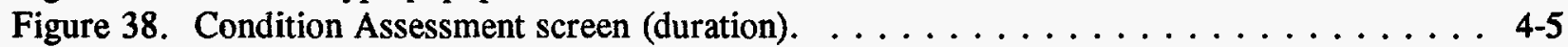

Figure 39. Select Sequence Sort window. . . . . . . . . . . . . . . . . . . 4-6

Figure 40. Uncertainty Calculation Values screen. . . . . . . . . . . . . . . . 4-7

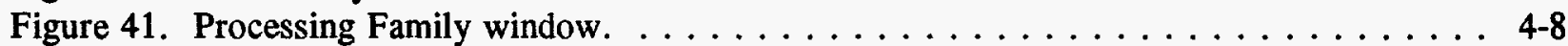

Figure 42. Uncertainty Results window. $\ldots \ldots \ldots \ldots \ldots \ldots \ldots \ldots \ldots \ldots$

Figure 43. Uncertainty Data screen. . . . . . . . . . . . . . . . . . . . . . 4 4-9

Figure 44. Current Quantile Values - example . . . . . . . . . . . . . . . . . . 4-9

Figure 45. Utilities screen. . . . . . . . . . . . . . . . . . . . 5-1

October $1995 \quad$ vii NUREG/CR-6116, Vol 6 
Figure 46. Hardware information, file locations, and archive information provided in the first Constants screen.

Figure 47. Cut set generation, transformations, uncertainty and miscellaneous settings for the second Constants screen. . . . . . . . . . . . . . . . . . . . 5-4

Figure 48. Fault tree graphics information for the third Constants screen. . . . . . . . . . . . . 5-6

Figure 49. Event tree graphics information for the fourth Constants screen. . . . . . . . . . 5-6

Figure 50. Archive Family screen. . . . . . . . . . . . . . . . . 5-7

Figure 51. Options window. . . . . . . . . . . . . . . . . . . . . . 5-9

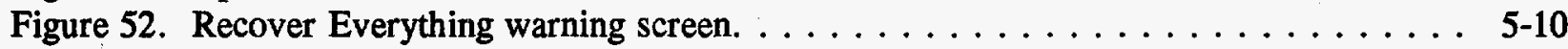

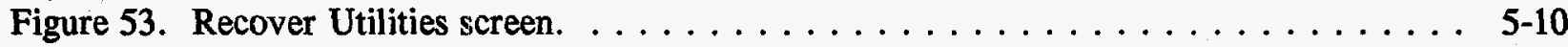

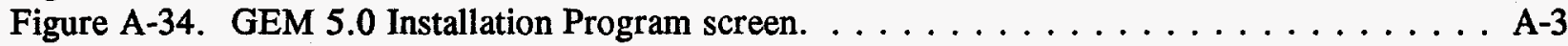

\section{TABLES}

1. User Information field descriptions for constants option $\ldots \ldots \ldots \ldots \ldots \ldots \ldots$

2. Default values and flag settings $\ldots \ldots \ldots \ldots \ldots \ldots \ldots \ldots \ldots \ldots \ldots$

C-1. LOOP Frequency Parameters $\ldots \ldots \ldots \ldots \ldots \ldots \ldots \ldots \ldots \ldots$

C-2. Seal LOCA cumulative distribution functions $\ldots \ldots \ldots \ldots \ldots \ldots \ldots$ 


\section{EXECUTIVE SUMMARY}

The Systems Analysis Programs for Hands-on Integrated Reliability Evaluations (SAPHIRE) refers to a set of several microcomputer programs that were developed to create and analyze probabilistic risk assessments (PRAs), primarily for nuclear power plants. The Idaho National Engineering Laboratory (INEL) has recently developed the Graphical Evaluation Model (GEM) as the U.S. Nuclear Regulatory Commission's (NRC's) state-of-the-art Accident Sequence Precursor (ASP) analysis tool. GEM is a special application tool using specifically-designed ASP SAPHIRE data bases to evaluate the conditional core damage probability (CCDP) of either operational occurrences or operating conditions at commercial nuclear power plants.

The INEL's role in GEM is to develop the software and interface to the user community, including training and technology transfer. The INEL is also the primary developer and maintainer of the ASP models that GEM uses. This is the first release of GEM, but it retains the version numbering of the SAPHIRE system since GEM shares the same database structure. This release contains all of the basic features required to perform CCDP analyses using the ASP methods. Future releases will incorporate enhancements as suggested by user comments and feedback.

GEM uses SAPHIRE plant models developed especially for ASP evaluations. These models contain unique recovery values dependent upon the initiating event of concern. GEM automates the application of these recovery values. For evaluation of conditions where an initiating event has not occurred, GEM automates the calculation of a CCDP based on the duration of the condition. For both initiating events and plant conditions, GEM simplifies the process of modifying the models for failed or out-of-service components and generating and quantifying the minimal conditional cut sets. 


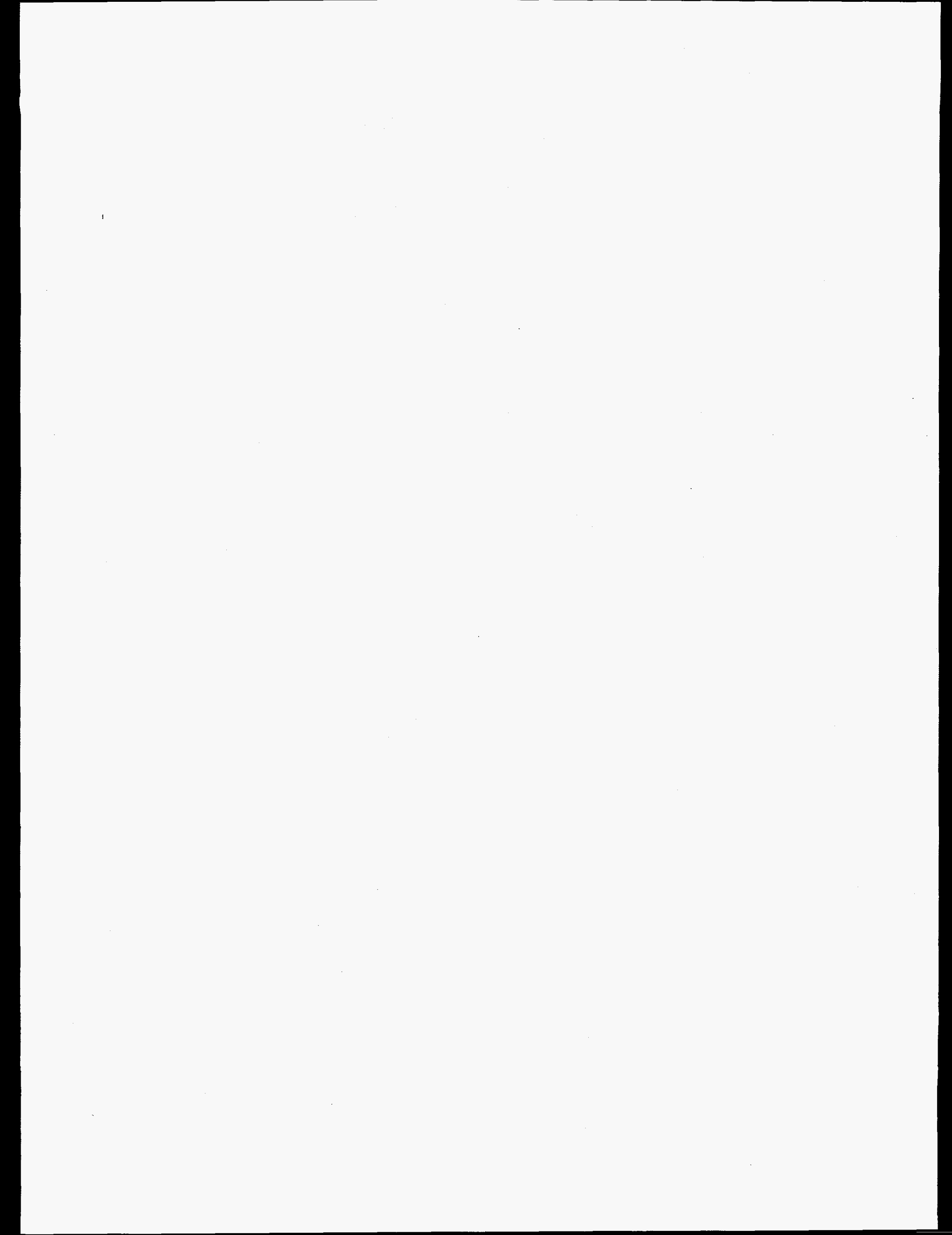




\section{FOREWORD}

The U.S. Nuclear Regulatory Commission has developed a powerful suite of personal computer programs for the performance of probabilistic risk assessments (PRAs). This suite of programs, known as the Systems Analysis Programs for Hands-on Integrated Reliability Evaluations (SAPHIRE), allows an analyst to perform many functions necessary to create, quantify, and evaluate the safety associated with the analysis of a facility or process. These programs include software to define the database structure; to create, analyze, and quantify the data; and to display results and perform sensitivity analyses. The programs included in this suite are the Models And Results Database (MAR-D) software, Integrated Reliability and Risk Analysis System (IRRAS) software, the Graphical Evaluation Module (GEM), Systems Analysis and Risk Assessment (SARA) software, and Fault tree, Event tree, and P\&ID (FEP) graphical editor software. Each of these programs performs a specific function in taking a PRA from conception to publication.

MAR-D is a program that is used primarily for PRA data loading. This program defines a common relational database structure that is used by the entire suite of programs. This structure allows all of the software to access and manipulate data created by other software in the system without performing a lengthy conversion. Therefore, data created by IRRAS is immediately available to GEM or SARA for safety analyses. The MAR-D program also provides the facilities for loading and unloading PRA data from the relational database structure used to store the data. MAR-D uses a simple ASCII data format to interchange data with other PRA software not included in NRC's suite of programs. This feature enhances compatibility with previously developed software systems and allows for maximum data interchange. Elements of this software are included with both IRRAS and SARA to enable these programs to load and unload data in the MAR-D format. Normally, only those who perform a data loading function use the entire MAR-D software; therefore, the end user does not require this software. Documentation for MAR-D Version 5.0 is available as NUREG/CR-6116, Volume 8. Please note that whenever the MAR-D database structure is changed, it necessitates changes in the remaining codes (i.e., IRRAS, SARA, GEM, and FEP). Therefore, the code version numbers are changed in unison. Each version set must be used together to maintain compatibility.

IRRAS performs those functions necessary to create and analyze a complete PRA. This program includes functions that allow the user to create event trees and fault trees, to define accident sequences and basic event failure data, to solve system and accident sequence fault trees, to quantify cut sets, and to perform uncertainty analysis on the results. IRRAS also lets the analyst generate reports and displays that can be used to document the results of an analysis. Because this software is a very detailed technical tool, the user of this program should be familiar with PRA concepts and the methods used to perform these analyses. Although IRRAS has been designed to be user friendly and makes the process of performing a PRA easier, the complexity of this type of analysis requires a user with a more detailed understanding of PRA concepts than is required by other tools in this suite. The IRRAS 5.0 reference manual and tutorial are available as NUREG/CR-6116, Volumes 2 and 3, respectively. In addition, a technical document that provides information on the principles and algorithms used in the construction and operation of IRRAS and SARA is available as NUREG/CR-6116, Volume 1. 
SARA is a program that allows the user to review the results of a PRA and to perform limited sensitivity analyses on these results. It is limited primarily to the extent that changes in the plant model can be accommodated only by using the cut set editor. If other than simple changes are being simulated, then IRRAS should be used so that new cut sets can be accurately generated and processed. This tool is intended to be used by a less technically-oriented user and does not require the level of understanding of PRA concepts required by IRRAS. With this program a user can review the information generated by a PRA analyst and compare the results to those generated by making limited modifications to the data in the PRA. Also included in this program is the ability to graphically display the information stored in the MAR-D database. This information includes event trees, fault trees, P\&IDs and uncertainty distributions. The user of this program can gain a better understanding of the PRA results without getting into the details of the construction and analysis work behind the PRA. The SARA reference manual and tutorial are available as NUREG/CR-6116, Volumes 4 and 5, respectively.

GEM provides a specific user interface tool for performing nuclear power plant operational event assessment using the SAPHIRE-based Accident Sequence Precursor (ASP) models and methods. GEM streamlines and automates select inputs and processes that are used to calculate conditional core damage probabilities (CCDPs) and provides outputs and reports consistent with ASP applications. For both initiating events and plant conditions, GEM simplifies the process of modifying the models for failed or out-of-service components and generating and quantifying the minimal conditional cut sets. With this program, a user can establish an analysis work area, make modifications to the model to represent the conditions of the operational event, and reprocess the models to automatically calculate the CCDP. This also includes the application or the appropriate recovery factors for the initiating event. For pressurized water reactors (PWRs), GEM also reevaluates the probability of a reactor coolant pump seal loss of coolant accident (LOCA). The GEM reference manual is available as NUREG/CR-6110, Volume 6.

FEP is a program developed to provide a common access to the suite of graphical tools developed for performing risk assessment. These tools include the graphical fault tree, event tree, and P\&ID editors. The fault tree and event tree editors are available through IRRAS; however, the P\&ID editor is only accessible through FEP. The fault tree editor allows the user to construct and modify graphical fault trees. The event tree editor allows the analyst to construct and modify graphical event trees. The P\&ID editor allows the user to construct and modify plant drawings. These drawings can then be used to document the modeling used in a PRA. These editors are an integral part of a PRA. With the FEP tool, the user need not be concerned with the complexity of the IRRAS program if the need is only to generate one of these graphical displays. The FEP Reference Manual is available as NUREG/CR-6116, Volume 7. 


\section{ACRONYMS}

ASCII

ASP

CCDP

DOS

FEP

F-V

GEM

ID

INEL

IRRAS

LOCA

LOOP

MAR-D

NRC

P\&ID

PC

PRA

PWR

SAPHIRE

SARA

American Standard Code for Information Interchange

Accident Sequence Precursor

conditional core damage probability

disk operating system

Fault tree, Event tree, and P\&ID code

Fussell-Vesely importance measure

Graphical Evaluation Module code

identification

Idaho National Engineering Laboratory

Integrated Reliability and Risk Analysis System code

loss of coolant accident

loss of off-site power

Models and Results Database code

U.S. Nuclear Regulatory Commission

piping and instrumentation diagram

personal computer

probabilistic risk assessment

pressurized water reactor

Systems Analysis Programs for Hands-on Integrated Reliability Evaluations codes Systems Analysis and Risk Assessment code 


\section{SAPHIRE Version 5.0}

\section{Volume 6-Graphical Evaluation Module (GEM) Reference Manual}

\section{INTRODUCTION}

The U.S. Nuclear Regulatory Commission (NRC) initiated the Graphical Evaluation Model (GEM) software development project, in response to a need for a personal-computer-based software to aid the NRC staff with evaluating initiating and conditional event failures in a timely manner. Specifically, a software package was needed that could readily calculate "what if" type event assessments on various fault tree models without changing the base-case values of these models. This package did not need to perform all of the detailed functions required of Integrated Reliability and Risk Analysis (IRRAS); however, it did need to provide certain essential functions such as modifying logic, generating cut sets, and quantifying cut sets. GEM 5.0 resulted from this software development project.

\subsection{GEM Attributes}

GEM uses SAPHIRE-based models developed in the Accident Sequence Precursor (ASP) program to manipulate, modify, and quantify processes. Designed for the IBM-PC and compatibles, GEM is readily accessible and portable. Taking advantage of new state-of-the-art algorithms, GEM is quite fast and powerful.

GEM simplifies the operational event analysis process and automates the construction of currentvalue input to the analysis software. The events in the models can be modified without changing the base-case values. GEM processes these events with IRRAS. GEM automatically applies new recovery action failure probabilities, depending on which initiating event is being analyzed, and calculates a new conditional core damage probability (CCDP). For conditions not involving an initiating event, GEM calculates a conditional core damage probability (CCDP) based on the modified basic event probabilities and the duration of the condition. The results pertaining to the CCDP are documented in various customized reports generated by the program. See Section B-2.1 for a definition of CCDP and other concepts.

The sections that follow provide the detailed information needed to become familiar with GEM 5.0. Section 1 provides an overview of this reference manual, specifics of hardware and software requirements, and installing GEM. Sections 2 through 5 present a step-by-step approach to using the basic capabilities of GEM.

NOTE: This document assumes that the user is familiar with PRA methods and terminology. This manual does not provide instruction on PRA methodology, but merely serves as a reference manual for the GEM user. 
Introduction

\subsection{Visual Font Styles}

The following conventions are used throughout this manual.

Font Style

ALL CAPITALS

Initial Capitals

Bold, ALL CAPITALS

$<$ Text $>$

$<$ Key $1>-<$ Key2 $>$

\section{Denotes}

Directory names and acronyms-for example directory IASPDB or acronym CCDP.

Menu items, command names, window names and options-for example Search and Report, Add Item command, and Plants.

Any command that must be typed-for example, A:INSTALL for installing the software.

The names of keys on your keyboard-for example, <Enter $>$, $<$ Home $>$, or $<$ Del $>$.

The minus sign (-) between key names indicates that you need to hold down the first key while you press the second key. For example, press $<$ Alt $>-<N>$ means to hold down the $<$ Alt $>$ key and press $<N>$. Then, release both keys.

\subsection{Function Key Designations}

The functions, located at the bottom of the option screens, send specific instructions to the system. Please note that function keys may vary depending upon which screen is being accessed.

$<\mathrm{F} 1>$

Help-Provides associated online help information for the option selected from the GEM main menu.

$<$ F5 $>$

Locate-Finds a specified event or family. Press $\langle$ F5 $>$ to access the screen. Enter all or part of the family or event name you wish to locate and press $<$ Enter $>$. This feature will place the highlight on the located name. If GEM does not locate the requested name, then the next name in alphabetical order will be highlighted. This feature is especially useful when several screens of families or events exist in the displayed list. 
Introduction

\subsection{System Requirements}

Before installing GEM, ensure that your system meets the following minimum hardware and software requirements:

- 386 processor, IBM-compatible PC with 2 MB of extended random access memory

- $\quad$ Laser or dot-matrix printer connected through port LPT1 (optional)

- $\quad 20 \mathrm{Mb}$ hard-disk drive with a minimum of $10 \mathrm{MB}$ of free space

- Floppy-disk drive

- $\quad$ Disk operating system (DOS) Version 3.3 or higher.

- $\quad$ Math co-processor (optional)

- 16 color enhanced monitor (EGA or better).

If an enhanced graphics adapter is used, it must have the memory expansion option to extend the standard 4 colors to 16. This option is an upgrade to the IBM EGA board, but is usually standard on boards manufactured by other vendors. GEM 5.0 does not support the 4-color mode on the EGA adapter.

The recommended configuration contains a VGA color monitor and adapter, a keyboard as the graphics input device, and a math co-processor. GEM will not run as fast if a math co-processor is not present.

NOTE: GEM requires the above specified amount of extended random access memory (RAM). This memory must be available for the program and not used by memory resident programs, such as "Side-Kick". Also certain memory resident programs may interfere with the execution of GEM 5.0. These should be unloaded before using GEM 5.0. The MS/DOS command "CHKDSK" can be used to display the amount of memory available on your PC.

\subsection{Installing GEM}

Appendix A contains the procedure for installing GEM 5.0 into your PC. The setup will automatically create the ISAF50ITOOLS directory, if it does not already exist. This directory takes. approximately $800 \mathrm{~K}$ of memory on the hard disk when first loaded. Storage requirements will increase as one uses the program and generates more data.

\subsection{Starting GEM}

Start GEM as follows:

1. Change directories to ISAF50 using CDISAF50,

2. Type GEM and press < Enter > to access the GEM main menu (Figure 1). 
Introduction

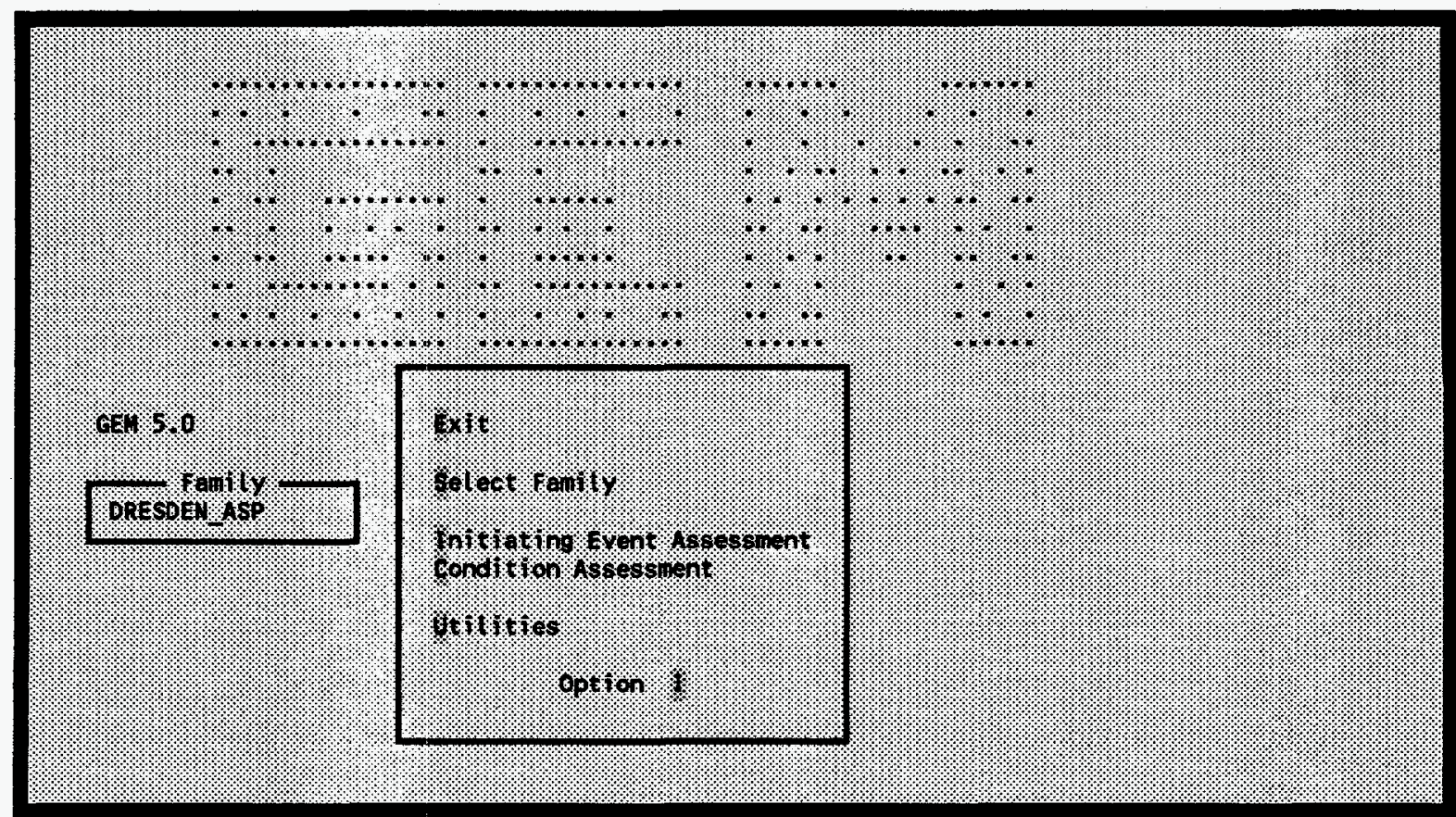

Figure 1. GEM main menu.

\subsection{GEM Options}

Select Family-Selects the family data set for analysis.

Initiating Event Assessment-Evaluates an operational occurrence associated with an observed initiating event. An initiating event assessment also considers the possible involvement of other equipment failures, unavailabilities, or degradations that impact the ability of the plant to properly respond to the initiating event.

Condition Assessment-Evaluates an operational occurrence in which no initiating event has taken place, but equipment failures, unavailabilities, or degradations have reduced the reliability of one or more systems to operate under off-normal conditions.

Utilities-Defines constants, archives databases, and recovers the database. Define Constants defines what hardware the system uses as well as specifying file locations and archive information, and defining uncertainty settings, cut set constants, and default values for the graphics editor. Archive Data Base compresses, backs up, restores, or expands data files for a family. Recover Data Base rebuilds damaged data files if the system seems to contain corrupted data or if the system keeps displaying error messages. You can also initiate the Recover Data Base at anytime to restructure and optimize the database to help the software run faster.

The information stored in the GEM database is buffered in and out of memory during processing. When the information is modified, GEM may not write the information out to the hard disk immediately. 
If one exits GEM in a means other than using the "EXIT" option from the main menu, then the new information may be lost. You should always exit GEM before turning off or rebooting your PC. If a power failure or abnormal program abort occurs, enter GEM, select the Utilities menu, and from that menu select the Recover Data Base option. Then, rebuild everything in the database before continuing to use the program on this data. Failure to recover the data base may cause unpredictable results. If GEM generates database results that cannot be explained, the database family in question should be rebuilt and the results checked again. If you still cannot explain the results, then the data base may contain a bug that is causing the error. Report the problem immediately to the system administrator.

\subsection{GEM Tools}

\subsubsection{Speed Search}

Use the speed search feature to facilitate your search. To use speed search, simply start typing the first few characters of the word you are trying to locate. As you type each letter, speed search simultaneously highlights the closest word that matches your entry.

\subsubsection{Marking Events}

You may mark some or all of the events in the list by using $\langle F 2\rangle,\langle F 3\rangle$, and $\langle F 4\rangle$. Asterisks will appear in front of all selected events. All three function keys function as a toggle switch, i.e., you can mark or unmark an event using the same key. For example, if you use $\langle$ F4 $\rangle$ to select a range of 10 event names, asterisks will appear in front of all event names that fall within the designated range. To remove the asterisks from the selected event names, simply press $\langle$ F 4$\rangle$ and select the same 10 event names for the range. Press $<$ F4 $>$ again and GEM will unmark the selected event names.

<F2 > Mark- Marks or unmarks a single event in a list.

<F3> Mark/Clr All-Marks or unmarks ALL events in a list.

<F4 $>\quad$ Sequentially marks or unmarks a range of event names in a list. To mark a range, highlight the first event name to include in the range and press $<F 4\rangle$. The message "Range selected-Highlight end of range..." appears at the bottom of the screen. Scroll through the list and find the last event name to include in the range and press $<$ F4 $>$. 


\section{SELECTING FAMILY}

Select Family selects the family data set you wish to analyze. A family consists of a group of models, such as those for a single plant, unit, or facility. When one selects a family, the Select Family screen will display the family name in the upper left corner of the screen.

The current directory is also the current family unless another family is selected. GEM retains the last family selected upon exiting the program. When GEM is entered again, the last family selected in the previous session becomes the current working family for the next session. The Select Family screen lists all GEM-compatible families in the SAPHIRE data base. See Figure 2 for an example of this screen.

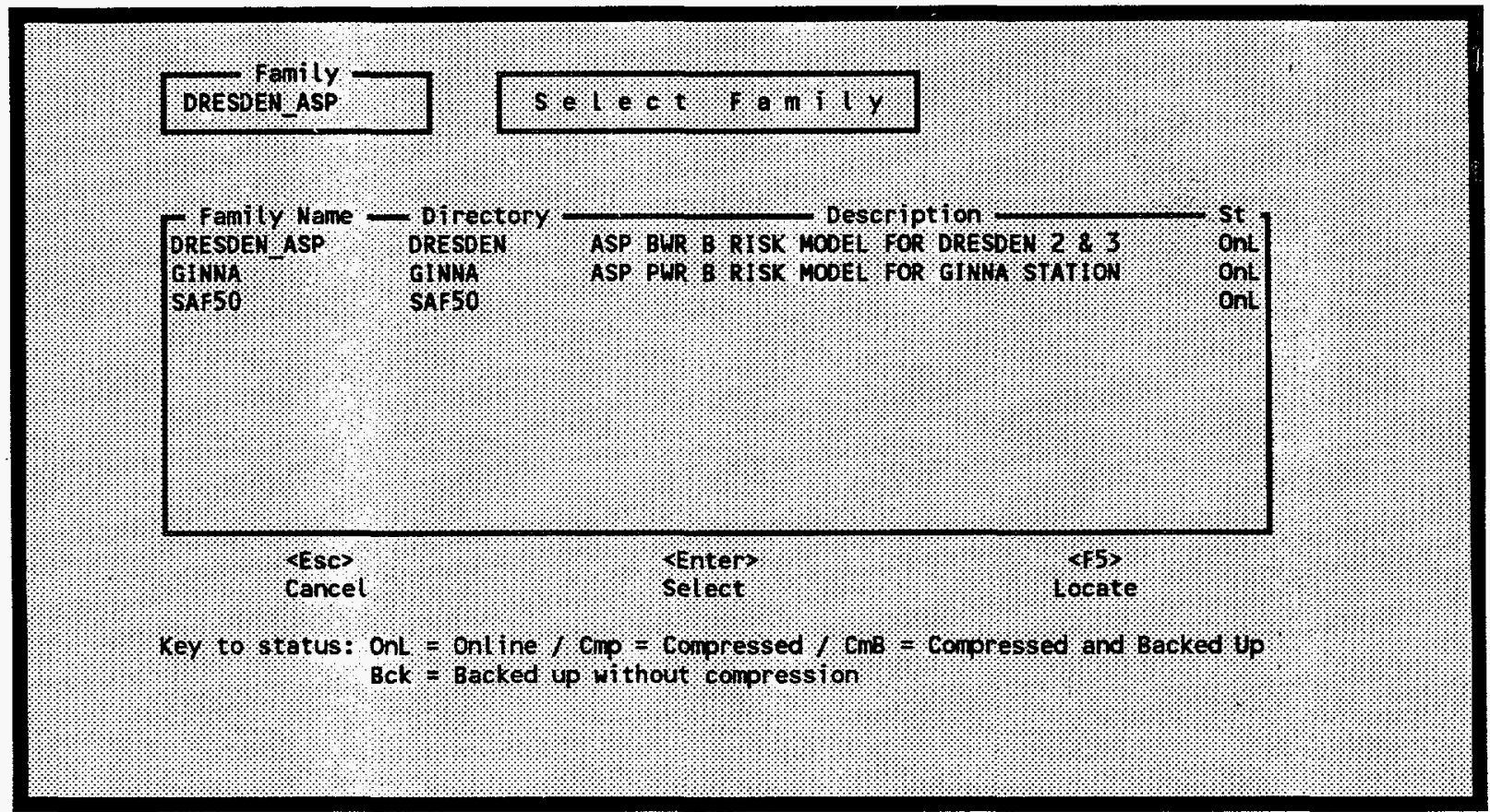

Figure 2. Select Family screen.

In addition, the screen provides a status field that shows the current state of the data base files for the family. The status field can contain any one of the following codes:

OnL-All files are online and can be accessed without any further action on your part.

Cmp-The data base files of the family are in a compressed state and are stored in an archive file. The compression packages PKZIP, LHA (LHARC), and ARJ are all supported. You must decompress these files before they can be accessed. Use the Archive Data Base option in the Utilities module to decompress these files (Section 5).

$\mathrm{CmB}$-The data base files of the family exist in a compressed state and are also backed up.

Bck-The family data base files were backed up without compression. 


\section{Select Family}

Select a family as follows:

1. Type $<S>$ or highlight Select Family using the up and down arrow keys.

2. Press <Enter $>$ to access the Select Family screen.

3. Highlight a family name and press <Enter $>$ to select the family and return to the main menu.

NOTE: $\quad$ Press $<$ Esc $>$ to exit the Select Family module and return to the main menu without changing the current family. 


\section{INITIATING EVENT ASSESSMENT}

An initiating event assessment evaluates an operational occurrence associated with an observed initiating event. An initiating event assessment also considers the possible involvement of other equipment failures, unavailabilities, or degradations that impact the ability of the plant to properly respond to the initiating event. This chapter discusses the following analysis steps to perform an initiating event assessment in detail. Refer to Section B-2.2.2 for a technical discussion of this topic.

1. Name Event ID - The Event ID equates to a unique identifier assigned to the particular operational event being analyzed. By using this identifier, you can readily return to a previously completed analysis or one that is in progress without losing any information.

2. Select an Initiating Event - GEM displays the list of available initiating events. Once you have selected the appropriate initiating event, GEM sets the frequency of the selected initiating event to the short-term recovery probability initiating event frequency and sets all other current event frequencies to 0.0 . You can either:

- Accept the initiating event recovery values and continue the analysis or

- $\quad$ Edit the recovery events to satisfy your current requirements.

3. Modify Data - This step allows you to stipulate the impact from the event analysis to the basic events of the model and determine the revised basic event values.

4. Process - This step takes the inputs that you just established and processes the SAPHIRE model to calculate the CCDPs for each of the appropriate accident sequences and the overall model. GEM generates the new basic event data, regenerates and requantifies the sequence minimal cut sets, and displays the results associated with the impacted sequences.

5. Results Display and Post-processing - You can sort the results and generate various reports, which are compatible with the ASP program. You can also list and partition the new sequence minimal cut sets as well as calculate and display the importance measures.

\subsection{Performing an Initiating Event Assessment}

Invoke the Initiating Event Assessment option as follows:

1. Type $<I>$ or highlight Initiating Event Assessment using the up and down arrow keys.

2. Press <Enter $>$ to access the Name Event ID screen (Figure 3).

\subsubsection{Name Event ID}

The Name Event ID screen allows you to assign a unique name, enter the date, and attach a description to the event analysis (Figure 3). 


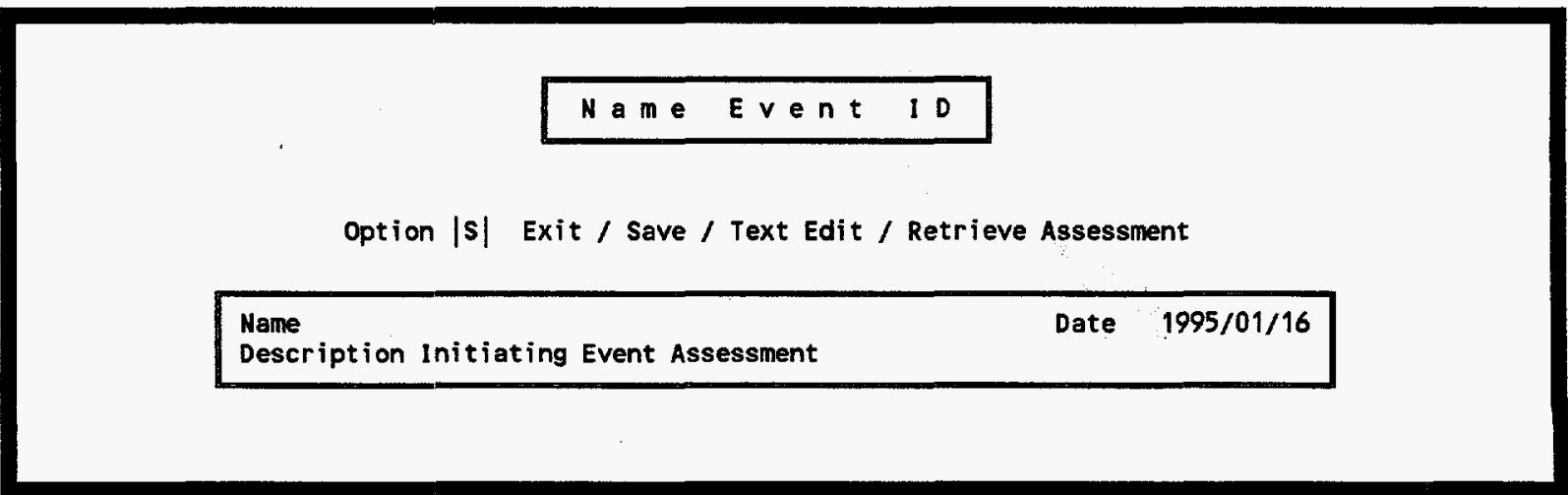

Figure 3. Name Event ID screen.

1. Assign a name to the event ID, using the Name field. If you leave the Name field blank, the message, "Enter an Event ID name" will appear at the bottom of the screen. To continue, enter a name for the event assessment.

2. (Optional) Tab to the Date field. You can change the default date by typing over the date. The date must remain in the same format presented in the field.

3. (Optional) Tab to the Description field. Enter a brief description of the event analysis. If you require more than the 60 -character event description provided in the Description field, you can attach an additional event description by typing a $\langle\mathrm{T}\rangle$ into the option field and pressing $<$ Enter $>$.

4. Place the cursor in the option field using the arrow keys.

5. Type $<S>$ into the Option field.

6. Press <Enter $>$ to save the event ID and access the Initiating Events screen (Figure 4). If a duplicate record exists in the database, a message at the bottom of the screen will ask if you want to overwrite the record. Type $\langle\mathrm{Y}\rangle$ to overwrite the record or $\langle\mathrm{N}\rangle$ to rename the event analysis.

\subsubsection{Select an Initiating Event}

Use the Select option to choose an initiating event. GEM will automatically set the other initiating event probabilities to zero. Note that GEM breaks down the loss of off-site power (LOOP) into four failure classes: grid-related, plant-centered, severe-weather, and extreme-severe-weather (Figure 4).

1. Highlight an initiating event by using the up and down arrow keys or use the speed search feature to locate an initiating event. See Section 1.8.1 for more details about speed search.

2. Choose either the Select or Edit Values option. 


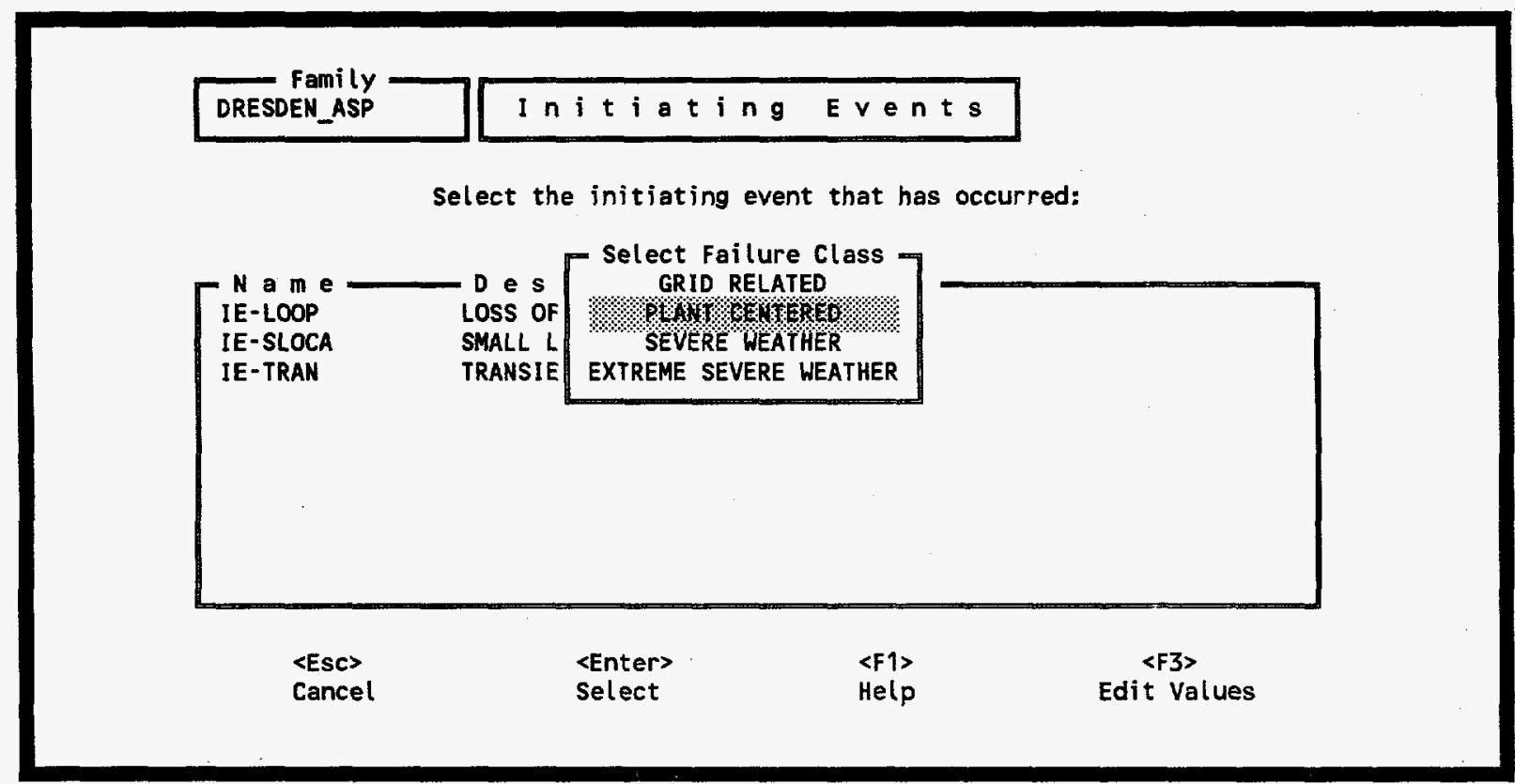

Figure 4. Select Failure Class window.

3.1.2.1 Select. Press < Enter $>$ to select the initiating event and access the Modify Data screen. See Section 3.1.3. If you select LOOP, the screen will display the Select Class window (Figure 4). Highlight the appropriate failure class and press <Enter $>$ to access the Modify Data screen.

3.1.2.2 Edit Values. The Edit Values function allows you to have the ability of modifying the recovery event before processing the event and continuing on to the next screen.

1. Press $\langle$ F3 $>$ to edit the nonrecovery value in the Recovery Event(s) screen (Figure 5).

If you select LOOP, the screen will display the Select Failure Class window (Figure 4).

a. Highlight the failure class.

b. Press $<$ Enter $>$ to access equivalent recovery event screen for the related LOOP, failure class (Figure 6).

NOTE: $\quad$ Press $<E s c>$ to return to the Select Class Failure window to either select another failure class or press $<\mathrm{Esc}>$ again to select another initiating event.

2. Highlight the initiating event by using the up and down arrow keys. Here, you can either process the default nonrecovery value or change the nonrecovery value before processing the initiating event. 


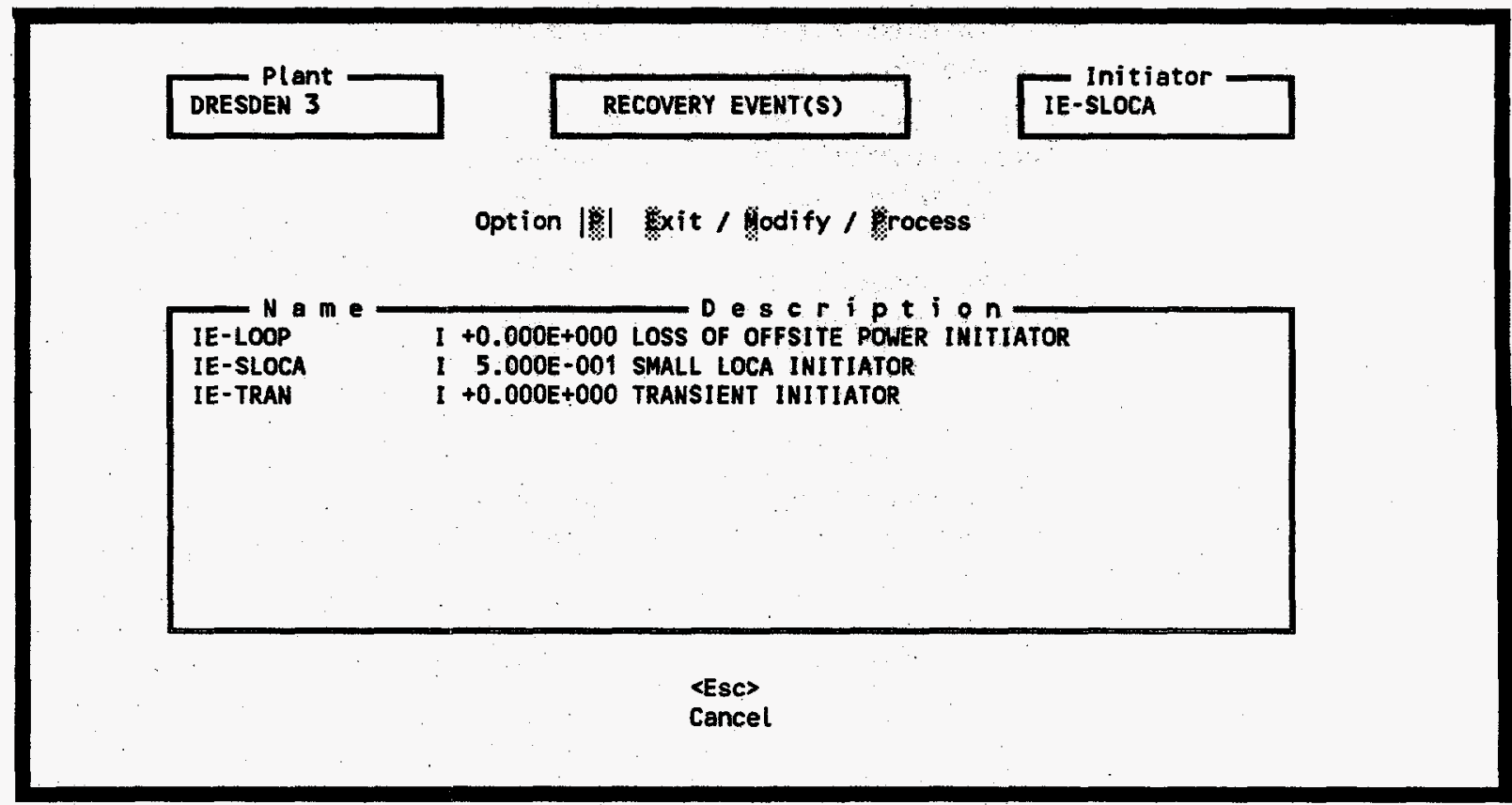

Figure 5. Recovery Event(s) screen for initiating events other than LOOP initiating events.

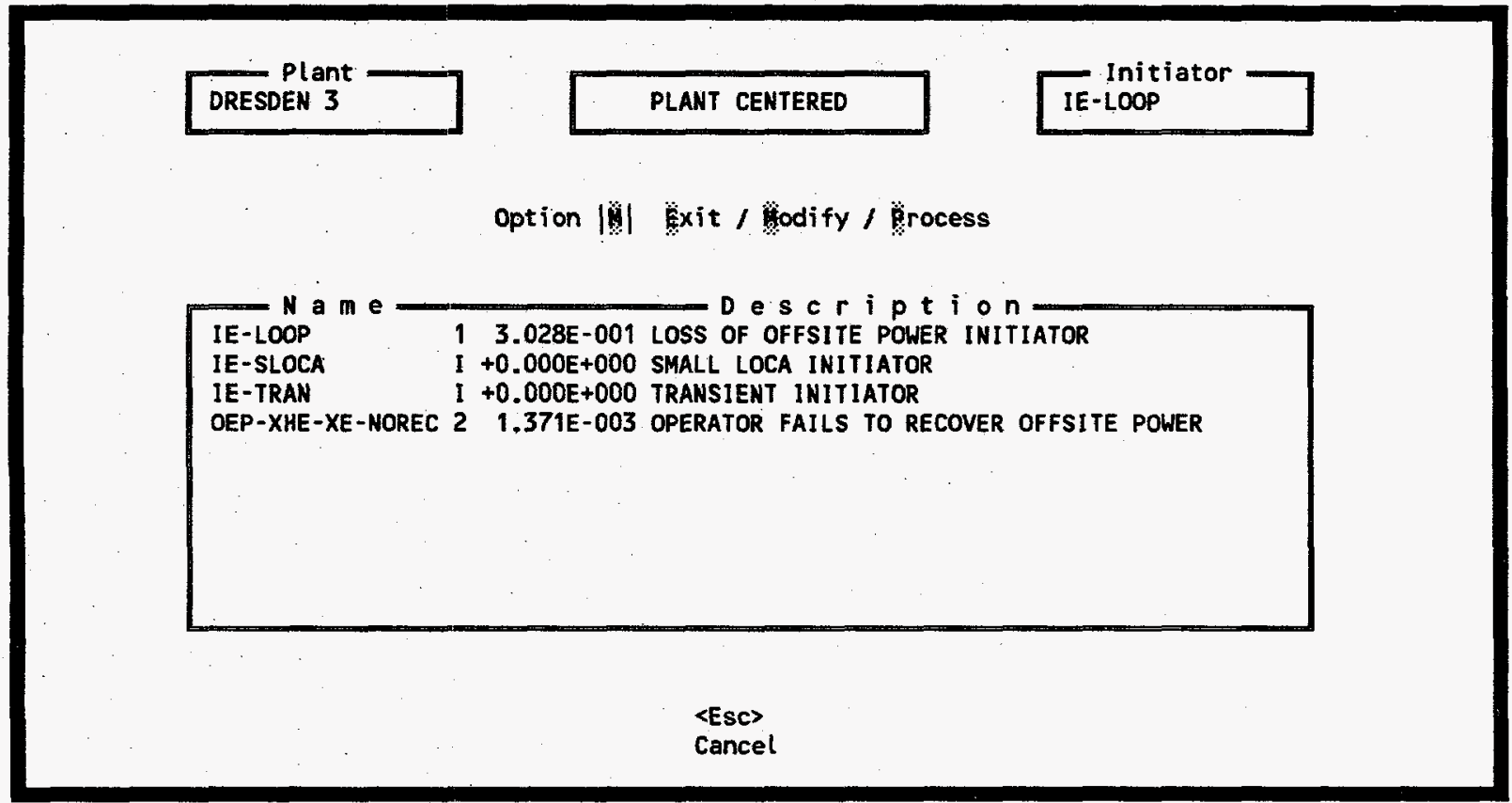

Figure 6. Example of a select failure class screen. 
a. Type $\langle\mathrm{P}\rangle$ and press $<$ Enter $>$ to process the default nonrecovery value and continue to the Modify Data screen. See Section 3.1.3.

b. Type $<M>$ and press $<$ Enter $>$ to change the nonrecovery value.

For the LOOP initiating event:

1). Tab to the various fields and enter the new values in the desired fields (Figure 7).

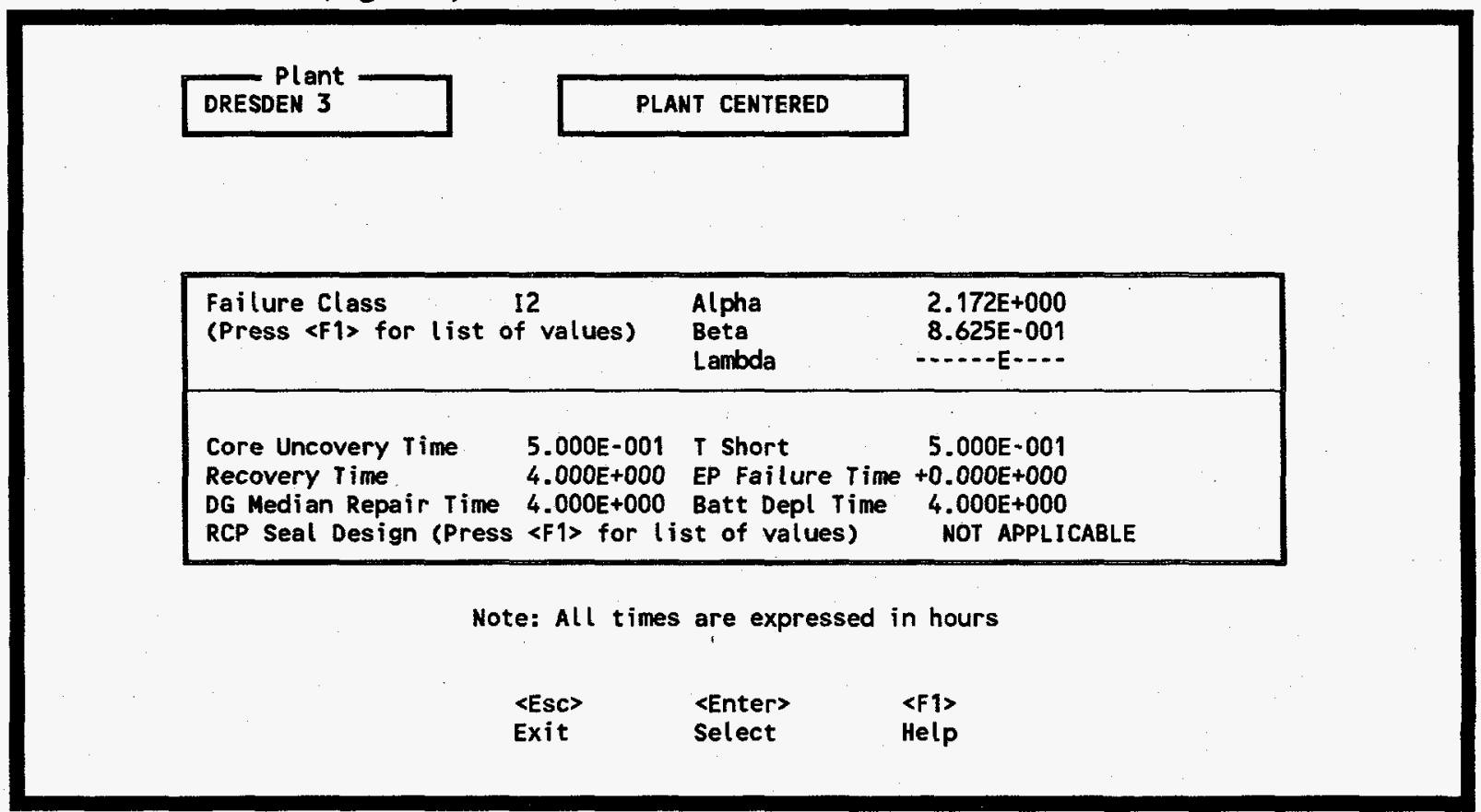

Figure 7. LOOP Nonrecovery Values screen

2). Press either $<$ Enter $>$ to save the changes and return to the previous screen or $\langle$ Esc $>$ to return to the previous screen without saving the changes.

3). Type $\langle P\rangle$ in the option field.

4). Press <Enter > to process the new nonrecovery value and continue on to the Modify Data screen. See Section 3.1.3.

For other initiating events:

1). Enter the new value in the Nonrecovery field (Figure 8).

2). Press either $<$ Enter $>$ to save the change and return to the previous screen or $<E s c>$ to return to the previous screen without saving the change.

3). Type $\langle P\rangle$ in the option field.

4). Press $<$ Enter $>$ to process the new nonrecovery value and continue to the Modify Data screen. See Section 3.1.3. 


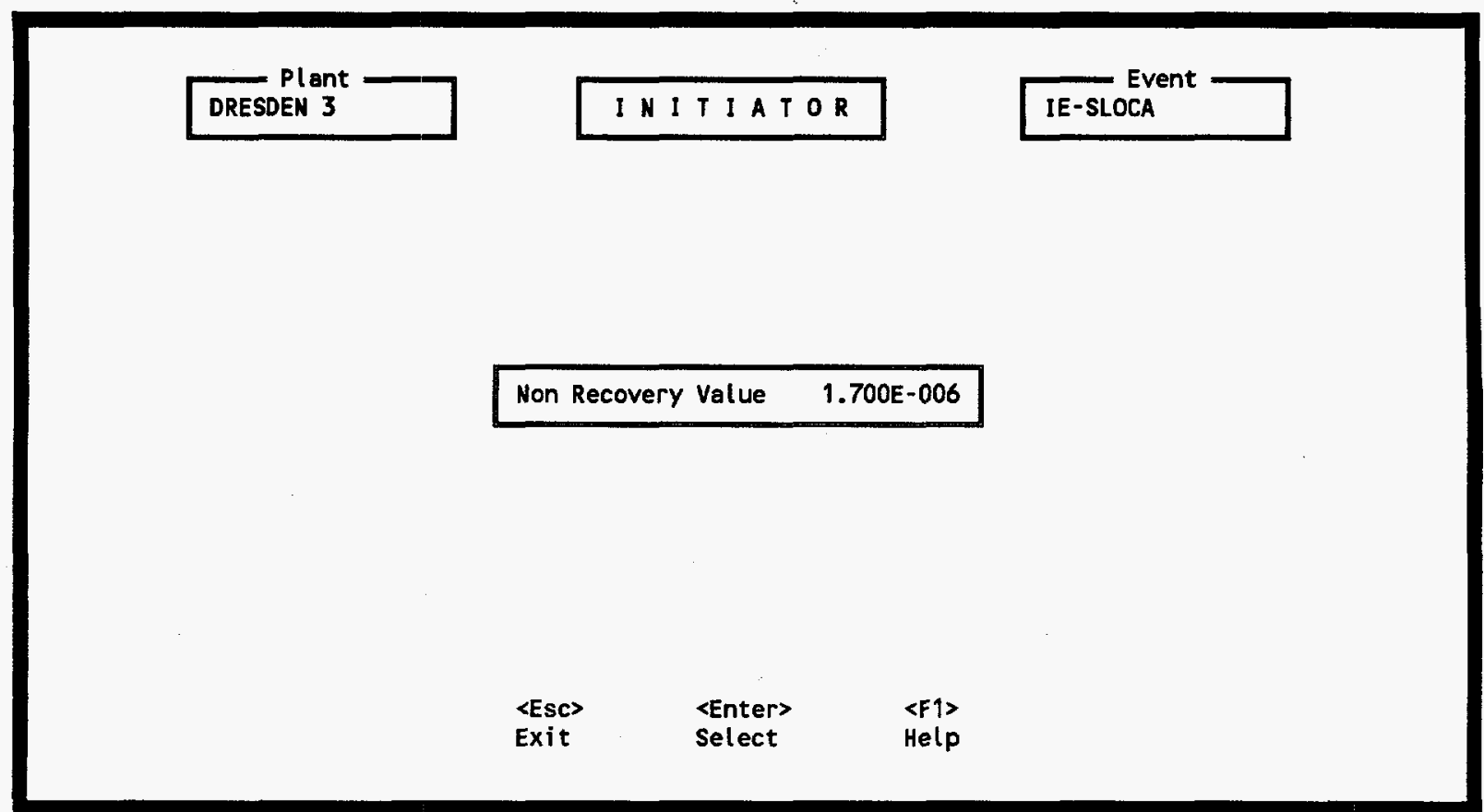

Figure 8. Other initiating events nonrecovery value screen.

\subsubsection{Modify Data}

Modify Data allows you to make changes to the existing list of initiating and basic events, add events to the list, change event values, copy an existing data set into the current data set, or reset an event to its base-case value. Process generates and quantifies current-case event data, generates new accident sequence minimal cut sets. GEM creates reports that reflect the event modifications that currently exist within the database. See Figure 9 for the Modify Data screen.

3.1.3.1 Add. Add adds an event or a group of events to the event ID.

1. Type $<A>$ in the option field.

2. Press $<$ Enter $>$ to access the Event window.

3. Either highlight an event using the arrow keys or use the speed search feature to locate and event name. You can use the $\langle F 2\rangle$ and $\langle F 4\rangle$ function keys to identify a group of basic events to assign a class change. See Sections 1.8.1 and 1.8.2 respectively for more details on speed search and marking text. Use $\langle\mathrm{F} 3\rangle$ to cancel all of the selected event names.

4. Press < Enter $>$ to access the Event Probability Changes screen. See Section 3.3 for details about this screen.

5. Tab to the various current-value fields and enter the changes in the desired fields. See Section 3.3 for details about the fields contained in the screen. 


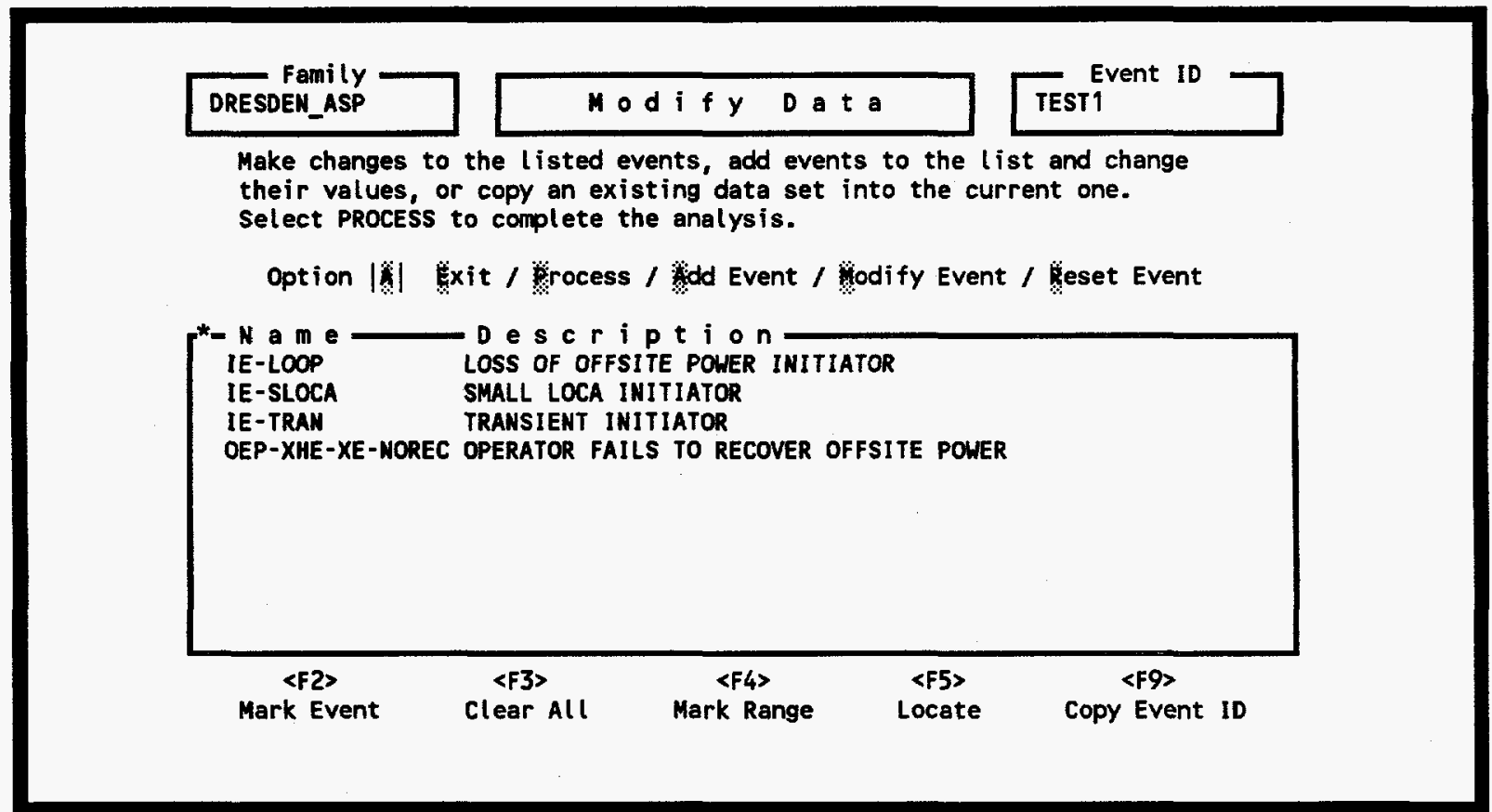

Figure 9. Modify Data screen.

6. Press <Enter > to add the event to the list of events in the Modify Data screen or press <Esc > to exit the Event Probability Changes screen and return to the Modify Data screen without saving the changes.

3.1.3.2 Modify Event. Modify Event gives you the ability to change current-case values for the events listed on the screen.

1. Highlight an event using the up and down arrow keys to change its current-case values or mark a group of events in the list to perform a class change.

2. Type $<M>$ in the option field to access the Event Probability Changes screen. See Section 3.3 for details about this screen.

3. Tab to the various current-value fields and enter the changes in the desired fields. See Section 3.3 for details about the fields contained in the screen.

4. Press $<$ Enter $>$ to modify the event and return to the Modify Data screen or press $<$ Esc $>$ to exit the Event Probability screen and return to the Modify Data screen without saving the changes.

3.1.3.3 Reset Event. Reset Event resets the current-case values for a selected event back to the base-case value of the event. 


\section{Initiating Event Assessment}

1. Highlight an event using the up and down arrow keys.

2. Type $\langle R>$ in the option field. The monitor will display the message "Reset HIGHLIGHTED probability change? (Y/N)."

3. Type $\langle\mathrm{Y}\rangle$ to reset the event values to the base-case values or $\langle\mathrm{N}\rangle$ to keep the current event values. By resetting an event back to its base-case value, GEM will remove the event from the list of modified events.

3.1.3.4 Copy Event ID. Copy Event ID copies the highlighted event ID into the current initiating event.

1. Press $\langle$ F9 $>$ to access the Copy Data screen (Figure 10).

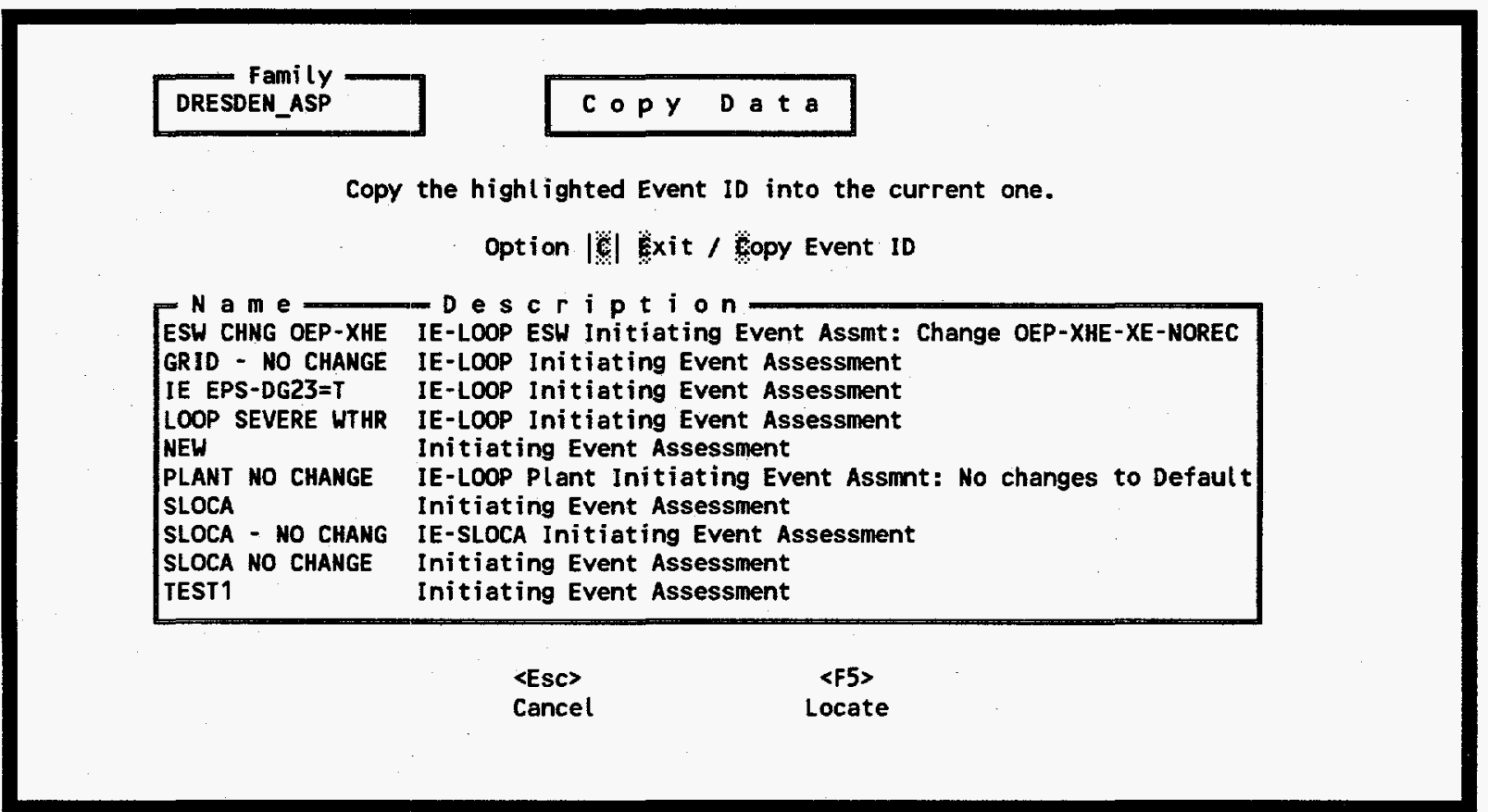

Figure 10. Copy Data screen.

2. Highlight the desired event ID using the up and down arrow keys.

3. Press <Enter $>$ to copy the event ID into the current one and return to the Modify Data screen.

3.1.3.5 Process. Process regenerates current case event data, loads and solves the logic for the associated accidental sequences, and creates and analyzes new cut sets for the affected sequences. After modifying any desired event, perform the following procedure.

1. Type $\langle P\rangle$ in the option field. 
2. Press <Enter $>$ to recalculate the new event data and to access the Event Assessment screen (Figure 11).

\subsubsection{Event Assessment}

Event Assessment displays the results pertaining to the CCDP. With these results, you can generate assessment reports for the event ID, display cut sets and sequence logic, partition cut sets, and resort the event tree sequences.

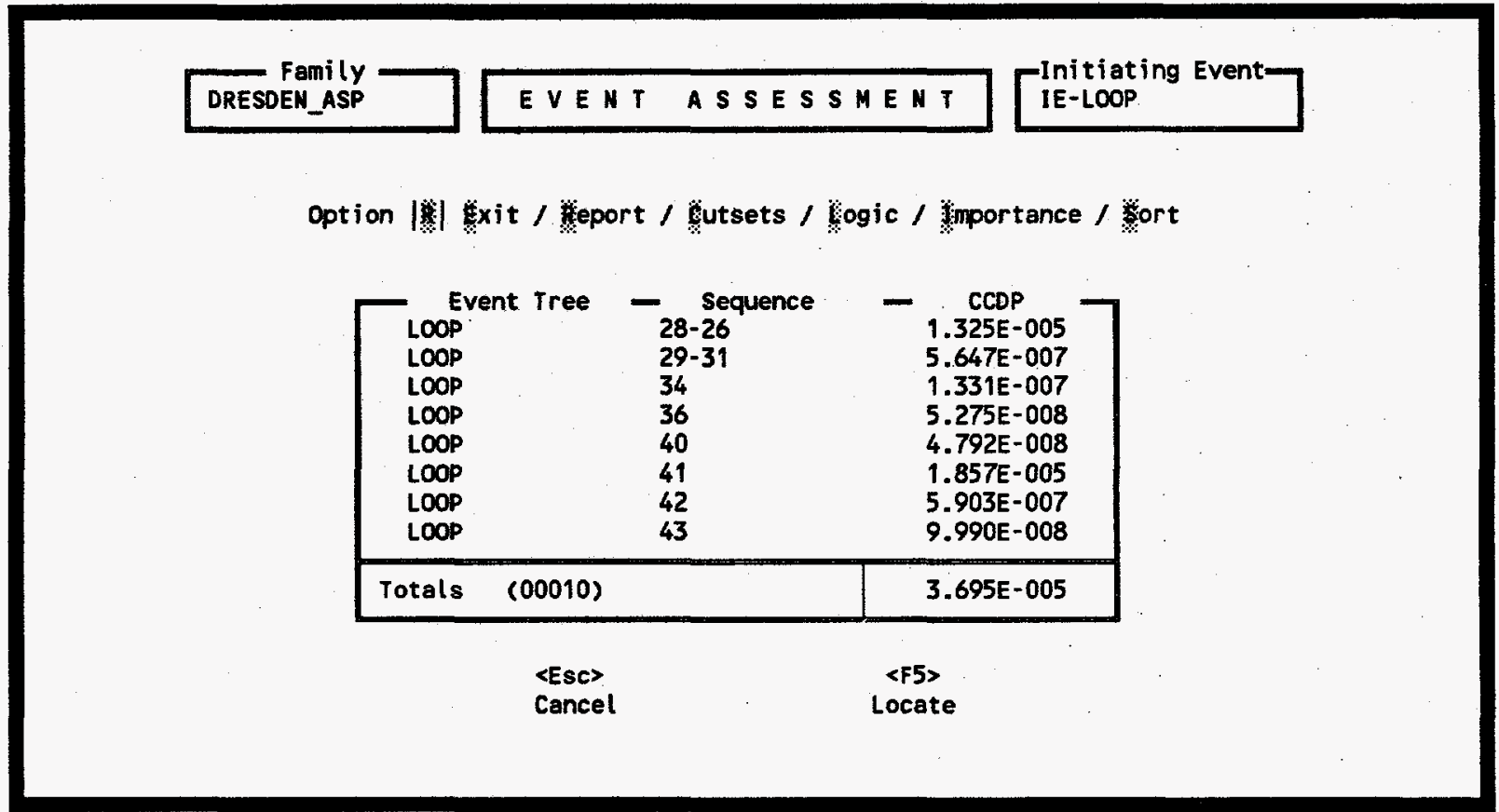

Figure 11. Event Assessment screen.

3.1.4.1 Exit and Escape. Exit returns to the main menu while escape returns to the previous screen.

Exit

1. Type $<E>$ in the option field.

2. Press $<$ Enter $>$ to exit the Event Assessment screen.

3. Your screen will display the message, "Return to the main menu?" (Y/N).

4. Type $\langle\mathrm{Y}\rangle$ to return to the Main menu or $\langle\mathrm{N}\rangle$ to remain at the Event Assessment screen. 
Initiating Event Assessment

Escape

1. Press $<$ Esc $>$.

2. Your screen will display the message, "Return to the previous menu?" (Y/N).

3. Type $\langle\mathrm{Y}\rangle$ to return to the Modify Data screen or $\langle\mathrm{N}\rangle$ to remain at the Event Assessment screen.

3.1.4.2 Report. Report generates Event Assessment reports for the event ID based on: basic event changes, sequence probabilities, sequence cut sets, and basic events (cut sets only or all basic events). Report allows you to generate the report in a WordPerfect 5.1 format, print a streamlined report that excludes page breaks and footers, truncate sequences and cut sets from the report based on contribution percentages to the CCDP, and select inclusion of just the basic events either used in cut sets or all basic events in the family. To generate a report, use the following procedure.

1. Highlight an event tree sequence by using the up and down arrow keys.

2. Type $\langle R\rangle$ in the option field.

3. Press <Enter $>$ to access the Reports screen (Figure 12). GEM provides a default report title; however, you may change this by simply typing over the existing text in the Report Title field.

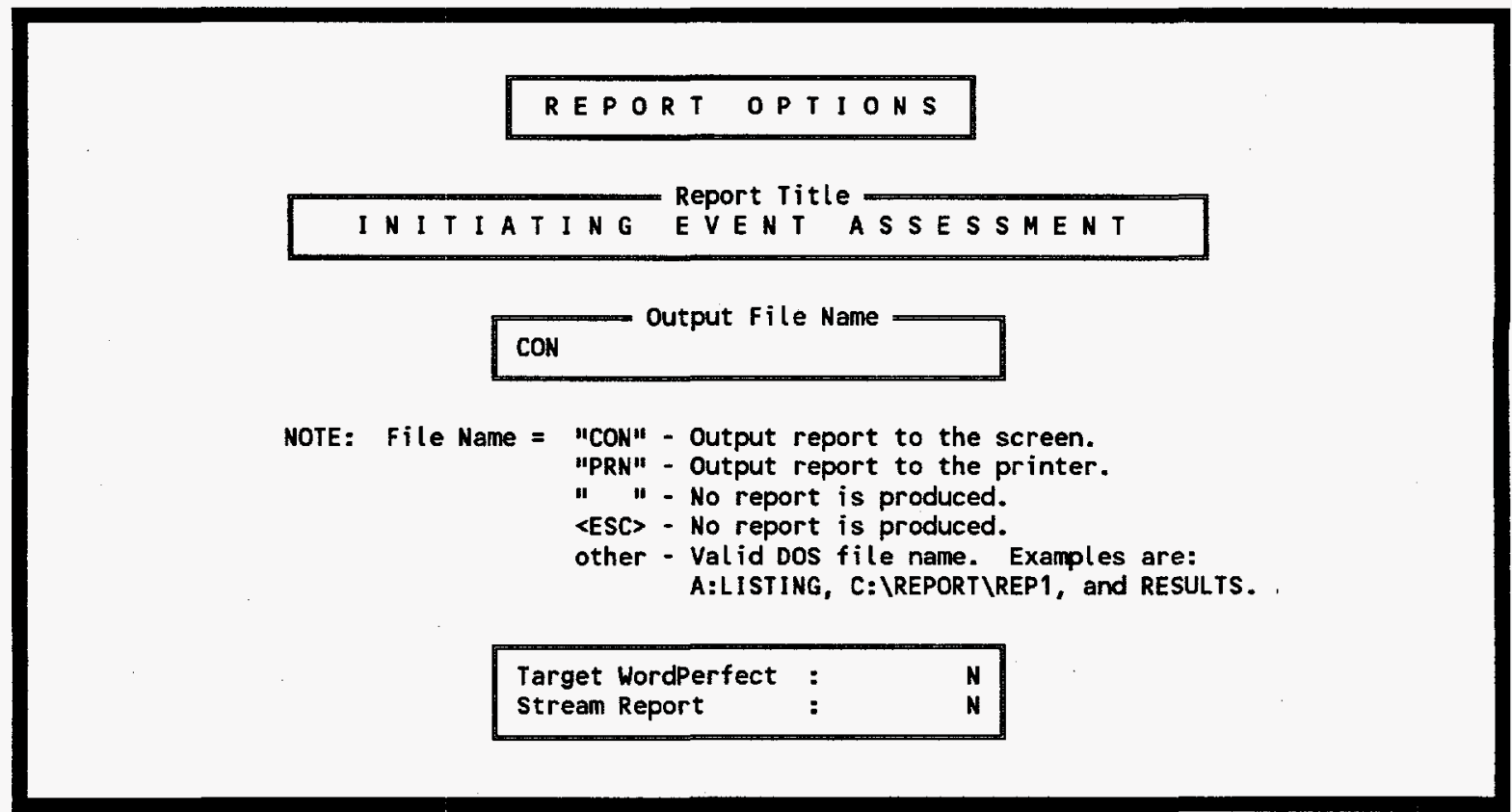

Figure 12. Reports main menu. 
4. Specify an output destination. As a default, the screen, denoted as CON, will display the report. However, you may change this to one of the following destinations:

PRN Prints the report on the attached printer.

$" \quad$ If the file name is left blank, no report will be generated.

$<$ Esc $>\quad$ No report will be generated.

other $\quad$ Enter a valid (DOS) file name. The report will be printed to that file. This file can then be printed later or transferred to another machine via a diskette.

5. (Optional) Type $\langle\mathrm{Y}\rangle$ in the Target WordPerfect field to print the report in a WordPerfect 5.1 format.

6. Type a valid DOS file name in the Output File Name field. If the DOS filename is missing, the screen will display the message, "WordPerfect format requires a valid file name."

7. (Optional) Type $\langle\mathrm{Y}\rangle$ in the Stream Report field to print out a report that excludes page breaks and footers.

8. Press $<$ Enter $>$ to display the next report criteria window (Figure 13).

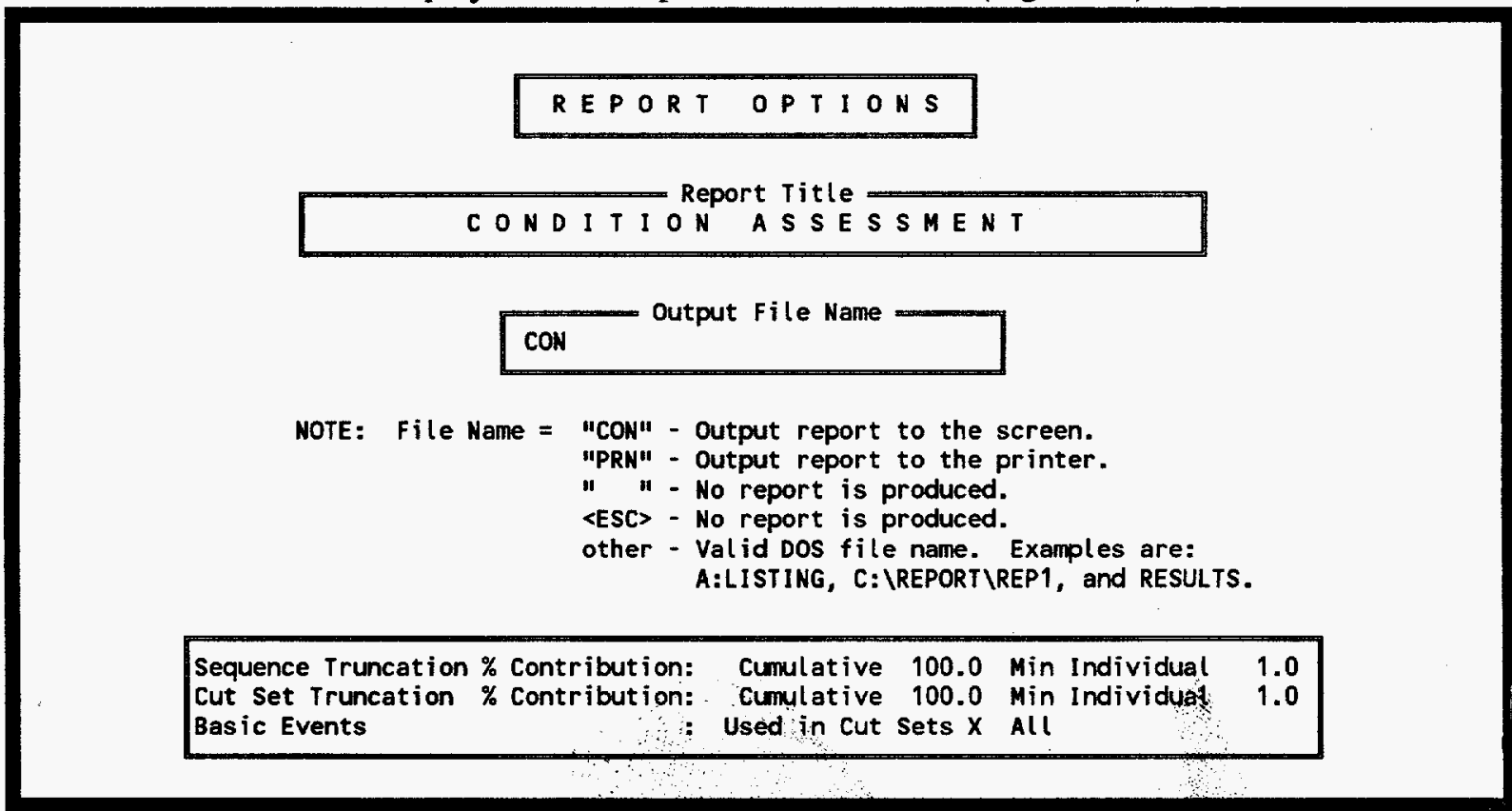

Figure 13. Sequence and Cut Set Contributions and Basic Event Parameters window. 


\section{Initiating Event Assessment}

9. (Optional) Tab to the event tree sequence and cut set contributions fields. You reduce the number of cut sets and sequences included in the report by setting the cumulative and minimum individual contribution fields.

a. Enter the total contribution and/or minimum percent contribution of event tree sequences and cut sets percentages to be included in the report. This will breviate the event sequences and cut sets printed in the report.

b. (Optional) Set the Min Individual fields to $1 \%$ if you want to work exclusively with the cumulative percentages.

c. (Optional) Set the Cumulative field to $100 \%$ if you want to work exclusively with the Min Individual fields percentages.

Example 1 helps explain how the cumulative feature works. This example displays the cut set events associated with sequences 35 and 36 of an initiating event. GEM adds the percent contribution of each cut set event in descending order until the percent contribution equals the value set in the Cut Set Truncation field. If the subtotal percent contribution $X_{i}$ is less than the desired total percent contribution $X_{t}$, and the addition of the next percent contribution $X_{i+1}$ causes the subtotal percent contribution $X_{i}$ to exceed the desired total percent contribution $X_{t}$ entered in the Cut Set Truncation field; then GEM will not add $X_{i+1}$ to $X_{t}$. In other words, if you enter 95 into the Cut Set Truncation field; then the report will only include the first cut set event since the addition of the second cut set event would cause the total percent contribution to exceed $95 \%$.

Example 2 displays only those contributions that exceed $5 \%$.

See Appendix $\mathrm{C}$ for two more examples.

10. Select either the basic events used in calculating the cut sets or all of the basic events in the family for the report.

11. Press $<$ Enter $>$ to initiate the report and follow the screen prompts displayed at the bottom of the screen. 
Initiating Event Assessment

Example 1: Cut set and sequence truncation (cumulative \% contribution) example.

INITIATING EVENT ASSESSMENT

Family :

DRESDEN_ASP

User Nane

Event ID :

TESTX

Description : Initiating Event Assessment

Model Ver :

Initiating Event : $\quad$ IE-SLOCA

Total CCDP :

$1.6 \mathrm{E}-005$

BASIC EVENT CHANGES

\begin{tabular}{ll} 
Event Name & Description \\
\hline IE-LOOP & LOSS OF OFFSITE POWER INITIATOR \\
IE-SLOCA & SMALL LOCA INITIATOR \\
IE-TRAN & TRANSIENT INITIATOR
\end{tabular}

\begin{tabular}{lll} 
Base Prob & Curr Prob & Type \\
\cline { 1 - 2 } $5.9 E-006$ & & $0.0 E+000$ \\
$1.7 E-006$ & $1.5 E+000$ \\
$3.4 E-004$ & $0.0 E+000$
\end{tabular}

SEQUENCE PROBABILITIES

Truncation: Cumulative: $95.0 \%$ Individual: $1.0 \%$

Event Tree Name

LOCA

Sequence Name

36

$\frac{\text { CCDP }}{1.5 E-005}$

$\frac{\text { \%Cont }}{93.8}$

Logic

System Name

RPS

Description

REACTOR SHUTDOWN FAILS

SEQUENCE CUT SETS

Truncation (\% cut Set) : Cumulative: $95.0 \%$ Individual: $1.0 \%$

Even Tree: LOCA

Sequence: 36

CCDP: 1.5E-005

Current \% Cut

CCDP Set

No cut sets to display

Cut Set Events 
Initiating Event Assessment

Example 2: Cut set and sequence truncation (minimum individual \% contribution) example.

IN IT I ATING EVENT ASSESSMENT

Family :

User Name :

Event ID :

DRESDEN_ASP

Description : Initiating Event Assessment

Model Ver :

IE-SLOCA

1.6E-005

BASIC EVENT CHANGES

$\begin{array}{ll}\text { Event Name } & \text { Description } \\ \text { IE-LOOP } & \text { LOSS OF OFFSITE POWER INITIATOR } \\ \text { IE-SLOCA } & \text { SMALL LOCA INITIATOR } \\ \text { IE-TRAN } & \text { TRANSIENT INITIATOR }\end{array}$

\begin{tabular}{|c|c|}
\hline $\begin{array}{l}\text { Base Prob } \\
5.9 E-006 \\
1.7 E-006 \\
3.4 E-004\end{array}$ & $\begin{array}{l}\text { Curr Prob } \\
0.0 E+000 \\
1.5 E+000 \\
0.0 E+000\end{array}$ \\
\hline
\end{tabular}

SEQUENCE PROBABILITIES

Truncation: Cumulative: $100.0 \%$ Individual: $5.0 \%$

Event Tree Name

LOCA

Sequence Name

36

$\frac{C C D P}{1.5 E-005}$

$\frac{\% \text { Cont }}{93.8}$

RPS Logic

LOCA

35

$1.0 E-006$

6.2 /RPS

PCS

MFW HCI

System Name

ADS

CRD

HCI

MFW

PCS

RPS

\section{Description}

AUTOMATIC DEPRESSURIZATION FAILS

INSUFFICIENT CRD FLOW TO RCS

HPCI FAILS TO PROVIDE SUFFICIENT FLOW TO RX VESSEL

FEEDWATER SYSTEM FAILS

POWER CONVERSION SYSTEM

REACTOR SHUTDOWN FAILS

SEQUENCE CUT SETS

Truncation (\% Cut Set) : Cumulative: $100.0 \%$ Individual: $5.0 \%$

Even Tree: LOCA

Sequence: 36

CCDP: $1.5 E-005$

\begin{tabular}{ll}
$\begin{array}{c}\text { Current } \\
\text { CCDP }\end{array}$ & $\begin{array}{c}\text { \% Cut } \\
\text { Set }\end{array}$ \\
\hline $1.5 E-005$ & 99.6
\end{tabular}

Even Tree: LOCA

Current

$\frac{\text { CCDP }}{4.5 E-007}$

1.7E-007

$1.7 E-007$

1.2E-007
\% Cut

$\frac{\text { Set }}{45.5}$

16.8

16.8

12.0
Cut Set Events

\begin{tabular}{|c|c|c|}
\hline \multicolumn{3}{|l|}{ RPS-SYS-FC-MECH } \\
\hline \multicolumn{2}{|c|}{ Sequence: 35} & CCDP: $1.0 E-006$ \\
\hline & Cut Set Events & \\
\hline $\begin{array}{l}\text { ADS-XHE-XE-ERROR } \\
\text { HCI-XHE-XE-NOREC } \\
\text { PCS-SYS-VF-MISC }\end{array}$ & $\begin{array}{l}\text { CRD-XHE-XE-ERROR } \\
\text { MFW-SYS-VF-FEEDW } \\
\text { PCS-XHE-XE-NOREC }\end{array}$ & $\begin{array}{l}\text { HCI - TDP-FC-TRAIN } \\
\text { MFW-XHE-XE-NOREC }\end{array}$ \\
\hline $\begin{array}{l}\text { ADS-XHE-XE-ERROR } \\
\text { HCI -TDP-FC-TRAIN } \\
\text { MFU-XHE-XE-NOREC }\end{array}$ & $\begin{array}{l}\text { CRD-MDP-FC-TRNB } \\
\text { HCI - XHE-XE-NOREC } \\
\text { PCS-SYS-VF-MISC }\end{array}$ & $\begin{array}{l}\text { CRD-XHE-XE-NOREC } \\
\text { MFH-SYS-VF-FEEDW } \\
\text { PCS-XHE-XE-NOREC }\end{array}$ \\
\hline $\begin{array}{l}\text { ADS-XHE-XE-ERROR } \\
\text { HCI -TDP-FC-TRAIN } \\
\text { MFW-XHE-XE-NOREC }\end{array}$ & $\begin{array}{l}\text { CRD-MDP-FC-TRNA } \\
\text { HCI-XHE-XE-NOREC } \\
\text { PCS-SYS-VF-MISC }\end{array}$ & $\begin{array}{l}\text { CRD-XHE-XE-NOREC } \\
\text { MFW-SYS-VF-FEEDW } \\
\text { PCS-XHE-XE-NOREC }\end{array}$ \\
\hline $\begin{array}{l}\text { ADS-SRV-CC-VALVS } \\
\text { HCI - TDP-FC-TRAIN } \\
\text { MFW-XHE-XE-NOREC }\end{array}$ & $\begin{array}{l}\text { ADS-XHE-XE-NOREC } \\
\text { HCI-XHE-XE-NOREC } \\
\text { PCS-SYS-VF-MISC }\end{array}$ & $\begin{array}{l}\text { CRD-XHE-XE-ERROR } \\
\text { MFW-SYS-VF-FEED } \\
\text { PCS-XHE-XE-NOREC }\end{array}$ \\
\hline
\end{tabular}


Initiating Event Assessment

BASIC EVENTS (Cut Sets Only)

\begin{abstract}
Event Name
ADS-SRV-CC-VALVS

ADS-XHE-XE-ERROR

ADS-XHE-XE-NOREC

CRD-MDP-FC-TRNA

CRD - MDP-FC-TRNB

CRD-XHE-XE-ERROR

CRD-XHE-XE-NOREC

HCI - TDP-FC-TRAIN

HCI -XHE-XE-NOREC

MFW-SYS-VF-FEEDW

MFW-XHE-XE-NOREC

PCS-SYS-VF-MISC

PCS-XHE-XE-NOREC

RPS-SYS- FC-MECH
\end{abstract}

\author{
Description \\ ADS VALVES FAIL TO OPEN \\ OPERATOR ERROR PREVENTS DEPRESSURIZATION \\ OPERATOR FAILS TO RECOVER ADS \\ TRAIN A FAILURES \\ TRAIN B FAILURE \\ OPERATOR FAILS TO ALIGN CRD \\ OPERATOR FAILS TO RECOVER CRD \\ HPCI TRAIN LEVEL FAILURES \\ OPERATOR FAILS TO RECOVER HPCI \\ FEEDWATER HARDWARE COMPONENTS FAIL \\ OPERATORS FAIL TO RECOVER FEEDWATER \\ PCS HARDWARE COMPONENTS FAIL \\ OPERATOR FAILS TO RECOVER PCS \\ MECHANICAL FAILURES OF THE RPS
}
Curr Prob
3.7E-003
1. $0 \mathrm{E}-002$
7.1E-001
3.7E-003
3.7E-003
$1.0 \mathrm{E}-002$
$1.0 E+000$
1.6E-001
7.1E-001
4.6E-001
3.4E-001
1.7E-001
$1.0 \mathrm{E}+000$
1. $0 \mathrm{E}-005$ 


\section{Initiating Event Assessment}

3.1.4.3 Cut Sets. The Cut Sets option generates the cut set list for the selected event tree sequence.

1. Highlight an event tree sequence by using the up and down arrow keys.

2. Type $\langle\mathrm{C}\rangle$ in the option field.

3. Press $<$ Enter $>$ to access the Cut Sets screen (Figure 14).

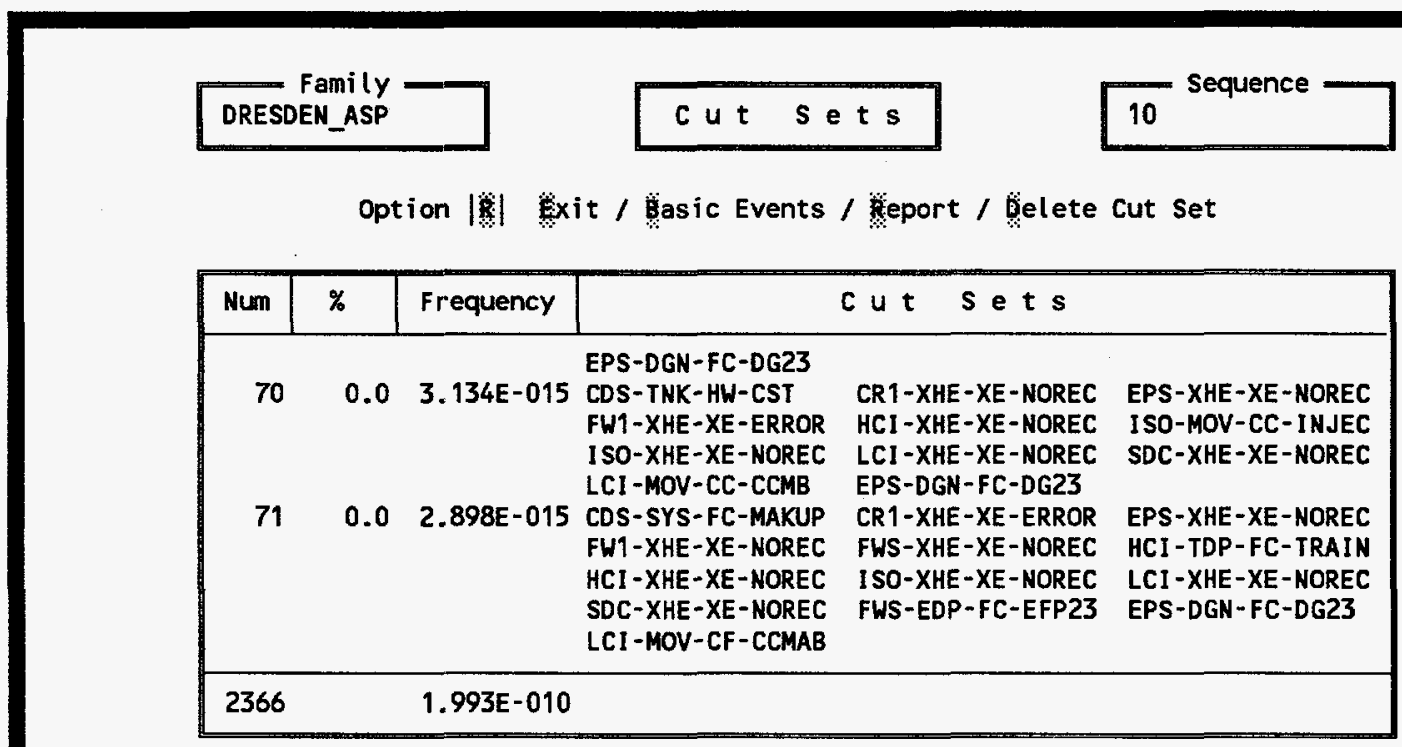

Figure 14. Cut Set screen.

3.1.4.3.1 Basic Events-Basic Events alphabetically lists the initiating event and event names making up the selected cut set and describes each event name and current probability.

1. Highlight a cut set in the list by using the up and down arrow keys.

2. Type $\langle\mathrm{B}\rangle$ in the option field.

3. Press $<$ Enter $>$ to access the Basic Events screen (Figure 15).

4. Use $<$ PgUp $>$ or $<\mathrm{PgDn}>$ to display more events.

5. Press $<$ Enter $>$ to return to the Cut Sets screen. 


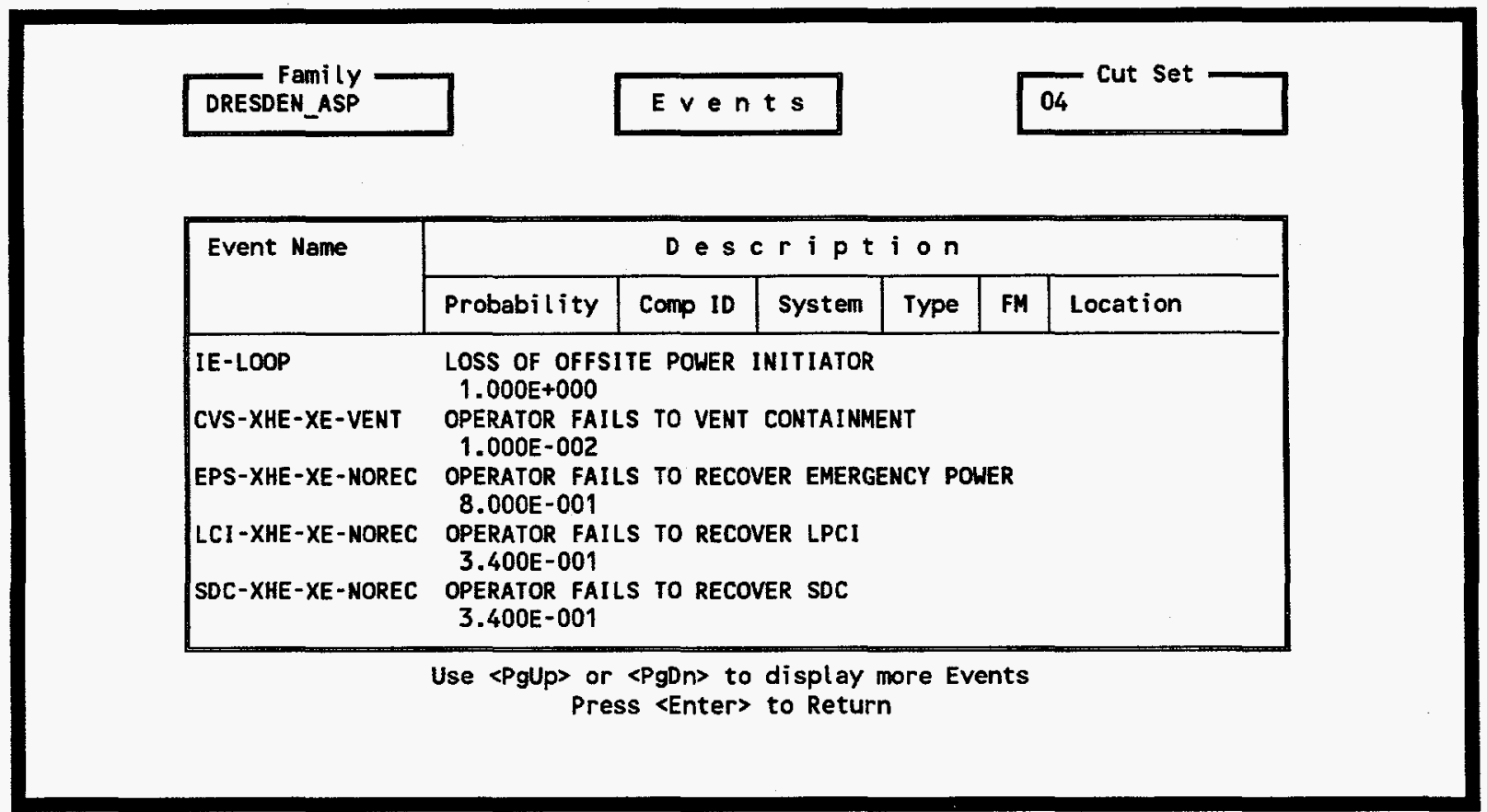

Figure 15. Basic Events screen.

3.1.4.3.2 Report-This option generates a Sequence Cut Set Report that consists of the event tree name, sequence number, sequence CCDP, current CCDP percent cut set contribution, and the cut set events. You can generate the report in a WordPerfect 5.1 format or print a streamlined report that excludes page breaks and footers. You can also define the cumulative percentage of cut sets or the percent contribution of each cut set for the report. See Section 3.1.4.2 for more details.

\subsection{Delete Cut Set-Delete Cut Set lets you remove cut sets from the list.}

1. Highlight a cut set in the list by using the up and down arrow keys.

2. Type $\langle D\rangle$ in the option field.

3. Press < Enter $>$ and GEM will display a confirmation of the cut set deletion at the bottom of the screen.

4. Type $\langle\mathrm{Y}\rangle$ to delete the cut set from the list or $\langle\mathrm{N}\rangle$ to cancel the cut set deletion.

3.1.4.4 Logic. Logic displays the sequence logic for the highlighted sequence. The Sequence Logic screen provides descriptions and names for the event tree, sequence, initiating event, and systems that compose the logic sequence. You can also print out the information in a report. GEM can print the report in a WordPerfect 5.1 format and/or print a streamlined report that excludes page breaks and footers. See Section 3.1.4.2 for more details. 


\section{Initiating Event Assessment}

1. Highlight an event tree sequence by using the up and down arrow keys.

2. Type $\langle L\rangle$ in the option field.

3. Press <Enter $>$ to access the Sequence Logic screen (Figure 16).

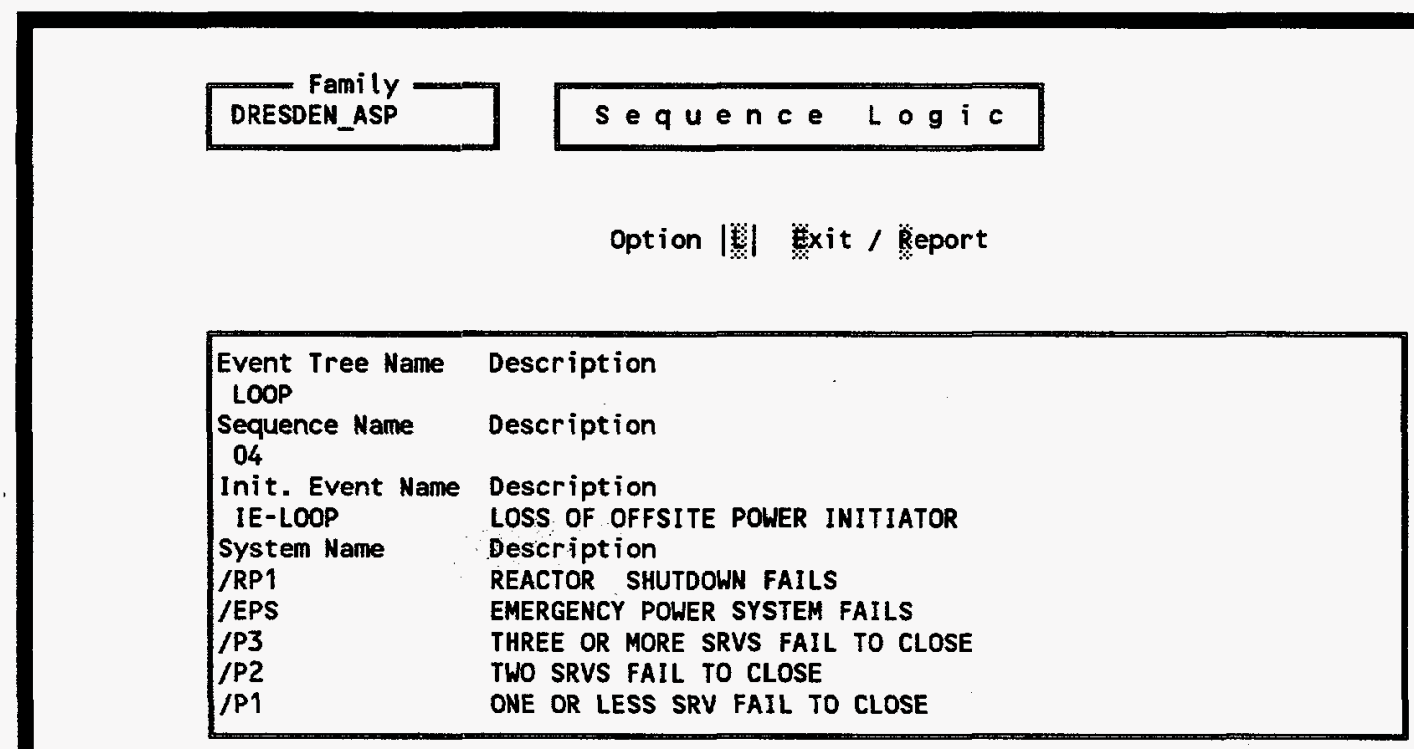

Figure 16. Sequence Logic screen.

3.1.4.4.2 Report-This option generates the Sequence Logic Report that consists of the event tree name, sequence name, sequence CCDP, current CCDP percent cut set contribution, and the logic. You can generate the report in a WordPerfect 5.1 format or print a streamlined report that excludes page breaks and footers. See Section 3.1.4.2 for more details.

3.1.4.5 Importance. Importance calculates the various importance measures within the capabilities of the Systems Analysis Programs for Hands-on Integrated Reliability Evaluations (SAPHIRE) based on the event assessment results. GEM sorts the events by the Fussell-Vesely (F-V) Importance Measure. The F-V importance calculates the fraction of the cut set upper bound (or sequence frequency) that involves the basic event of concern. Initiate the importance option as follows.

1. Type $<I>$ in the option field.

2. Press $<$ Enter $>$ to access the Importance screen (Figure 17).

The Importance option describes the event name, allows you to partition the cut sets, print out a report, and resort the events. Initiate the features within the Importance option as follows. 


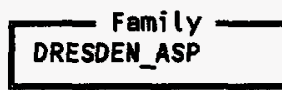

I mportance

- Cut Set Name GROUP

Option |

\begin{tabular}{|c|c|c|c|c|c|}
\hline Event Neme & \# of & Probability & $F-V$ & Ri & Risk I \\
\hline IE-LC & 136 & 3.02 & 1.00 & & $3.302 \mathrm{E}$ \\
\hline HCI - XHE-XE-NOREC & 36902 & 01 & .001 & $7.873 E+000$ & $1.357 \mathrm{E}$ \\
\hline HCI-TDP-FC-TRAIN & 20366 & $E-001$ & $8.727 E-001$ & 000 & $581 E+000$ \\
\hline PPR-SRV- $00-1 \mathrm{VLV}$ & 609 & 3.6 & $8.582 E-001$ & & -000 \\
\hline EPS-XHE-XE-NOREC & & & $8.299 E-001$ & & 1.207 \\
\hline EPS-DGN-FC-DG23 & 743 & $7.800 E-002$ & 4.093E-001 & $1.693 E+000$ & $5.838 E+000$ \\
\hline EPS-DGN-CF-DGNS & 91 & $E-003$ & $3.996 \mathrm{E}$ & 1.6 & $2.861 E+002$ \\
\hline CRD - XHE-XE - NOREC & 10 & 1.00 & & & $1.000 E+000$ \\
\hline & & & & & \\
\hline EP & 74 & $.800 E-0$ & 1 & 0 & bo \\
\hline
\end{tabular}

Sorted by F-V...

Figure 17. Importance screen.

3.1.4.5.1 Exit-Exit returns to the main menu.

1. Type $\langle E\rangle$ in the option field.

2. - Press $<$ Enter $>$ to exit the Importance screen.

3. Your screen will display the message, "Return to the previous menu?" (Y/N).

4. Type $\langle Y\rangle$ to return to the Modify Data screen or $\langle N\rangle$ to remain at the Event Assessment screen.

3.1.4.5.2 Description-This option describes the selected event.

1. Highlight an event name using the up and down arrow keys.

2. Type $\langle D\rangle$ in the option field.

3. Press $<$ Enter $>$ to display the event description at the bottom of the screen.

3.1.4.5.3 Partition-This option displays only a subset of the minimal cut sets or basic events that meet your requirements defined in subsequent screens. Partition customizes the cut sets by including events in the cut set and/or excluding events from the cut set. Partition also allows you to view the events. Invoke this option as follows.

October 1995 
Initiating Event Assessment

1. Type $\langle\mathrm{P}\rangle$ in the option field.

2. Press $<$ Enter $>$ to access the Partition screen (Figure 18).

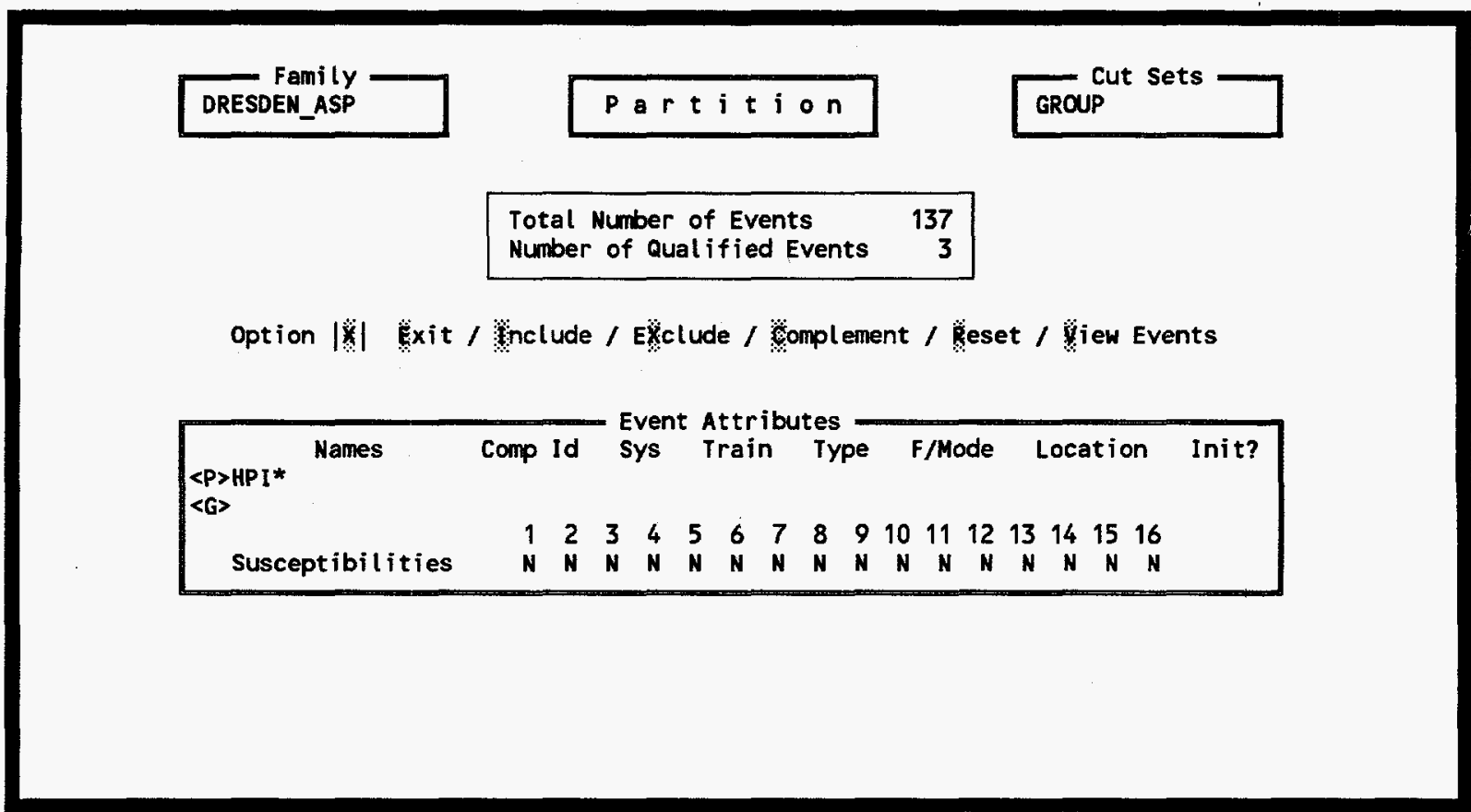

Figure 18. Partition screen.

Include-Include adds an event or a group of events to the partition. Establish a partition via this option as follows.

a. Type $<I>$ in the option field.

b. Fill the desired entry fields for qualifying the events that may be used in the new partition.

c. Press < Enter $>$ to qualify the events. After completing this process, GEM will update the Number of Qualified Events field that appears at the top of the screen. Here, qualified events are those events that contain the included attributes.

d. Return to the Importance screen via the Exit option. You will see that the system cut set list contains only those cut sets that are made up of qualified events. Figure 19 was the result of specifying the event name "HCI*" for the "Include" option. If the system cut sets do not contain any of the qualified events, then the screen will display the message, "No cut sets qualify."

NOTE: $\quad$ You can specify wildcards for the $\langle\mathrm{P}\rangle$ (primary) and $\langle\mathrm{G}\rangle$ (group) fields. The wildcards equate to those wildcards used in DOS. That is, an asterisk $\left(^{*}\right)$ represents an entire word or a group of characters. For 


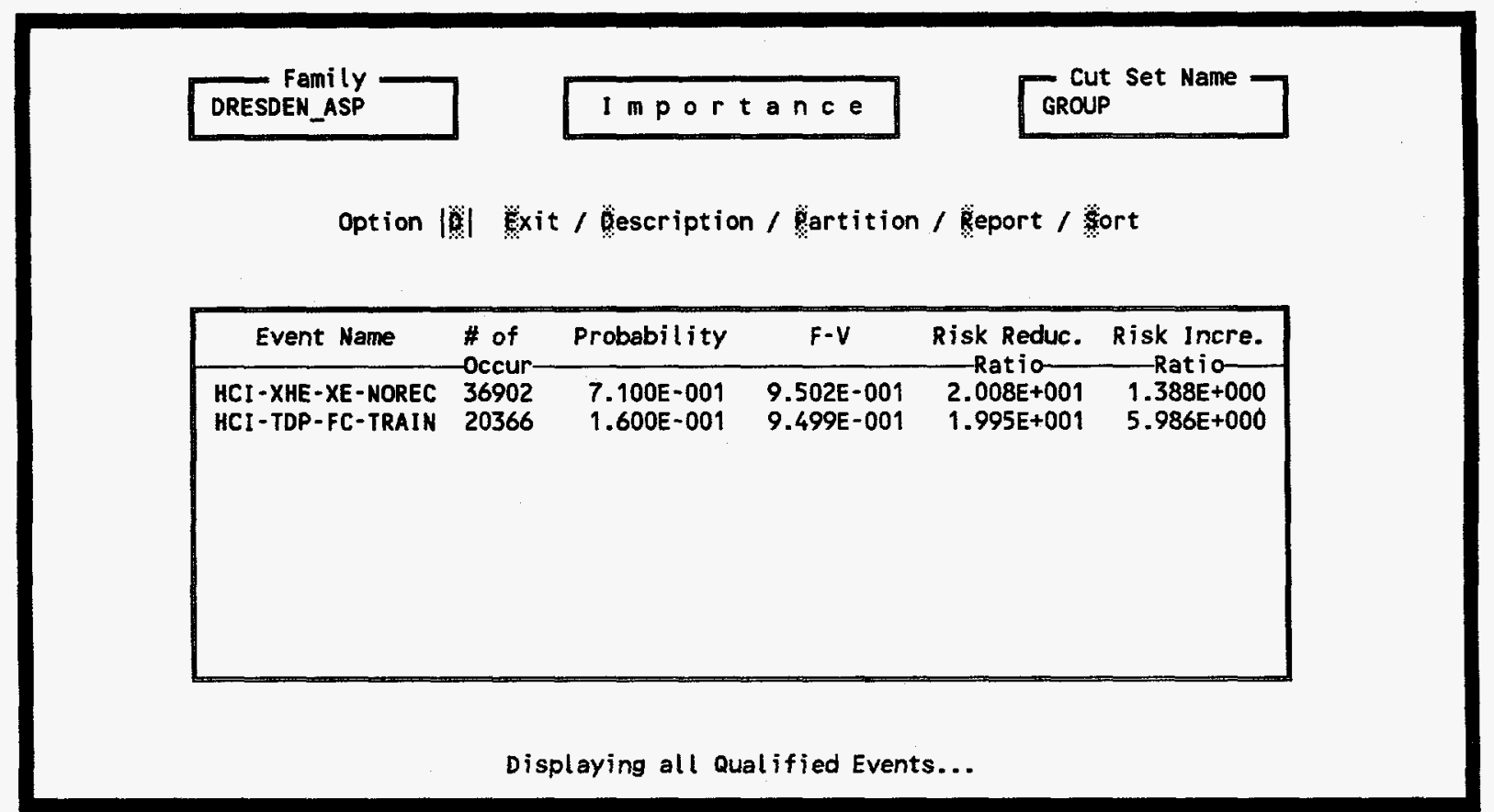

Figure 19. Example of the Importance screen using the Include option.

example, entering an $\mathbf{H C I} *$ in the $\langle\mathrm{P}\rangle$ or $\langle\mathrm{G}\rangle$ field will locate the event names HCI-XHE-XE-NOREC and HCI-TDP-FC-TRAIN in the DRESDEN_ASP family.

Exclude-Exclude creates a partition by deleting an event or a group of events from the list of events. Establish a partition via this option as follows.

a. Type $\langle X\rangle$ in the option field.

b. Fill the desired entry fields for removing the events from the list of qualified events.

c. Press $<$ Enter $>$ to remove the events. After completing this process, GEM will update the Number of Qualified Events field that appears at the top of the screen.

d. Return to the Importance screen via the Exit option. You will see that the system cut set list contains only those cut sets that are made up of qualified events. Figure 20 was the result of specifying the event name "HCI-TDP-FC-TRAIN" for the "EXclude" option. If the system cut sets do not contain any of the qualified events, then the screen will display the message, "No cut sets qualify."

Complement-Complement disqualifies all currently qualified events and qualifies all unqualified events. Establish a partition via this option as follows. 


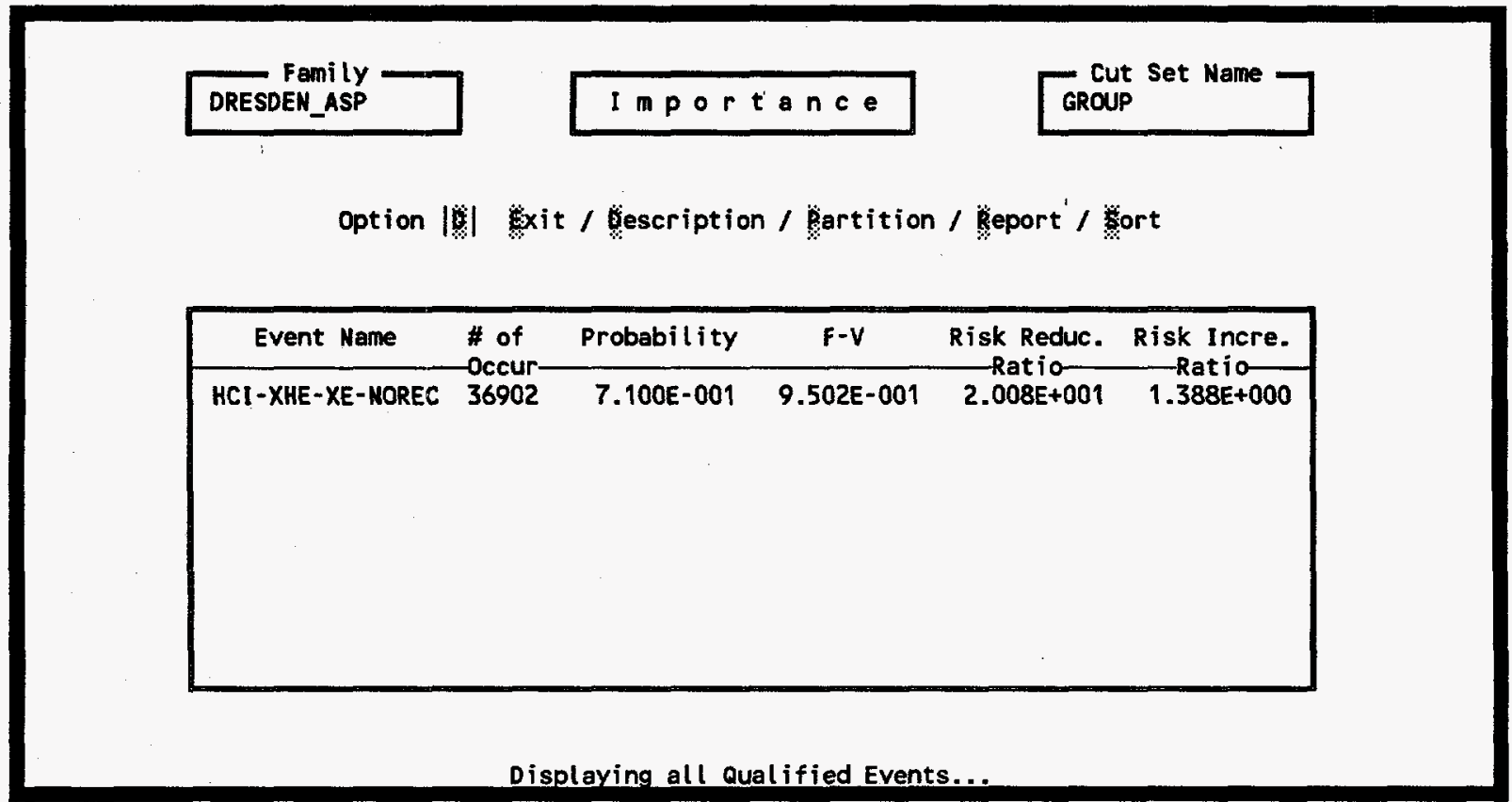

Figure 20. Example of the Importance screen using the Exclude option.

a. Type $<\mathrm{C}>$ in the option field.

b. Press <Enter $>$ to complement the qualified and disqualified events. The Number of Qualified Events field at the top of the screen will change accordingly.

c. Return to the Cut Sets screen via the Exit option. You will see that the system cut set list contains only those cut sets that are made up of qualified events (Figure 21). If the system cut sets do not contain any of the qualified events, then the screen will display the message, "No cut sets qualify."

Reset-Reset sets all events in the data base to qualified. This will remove all partitioning from the current system cut sets. Activate this option as follows.

a. Type $<R>$ in the option field.

b. Press $<$ Enter $>$. The original cut set list will result.

View Events-View Event displays the list of family events and allows you to mark those events that you want considered as qualified events. Activate this option as follows.

a. Type $\langle\mathrm{V}\rangle$ in the option field.

b. Press $<$ Enter $>$ to access the View Events screen (Figure 22). 


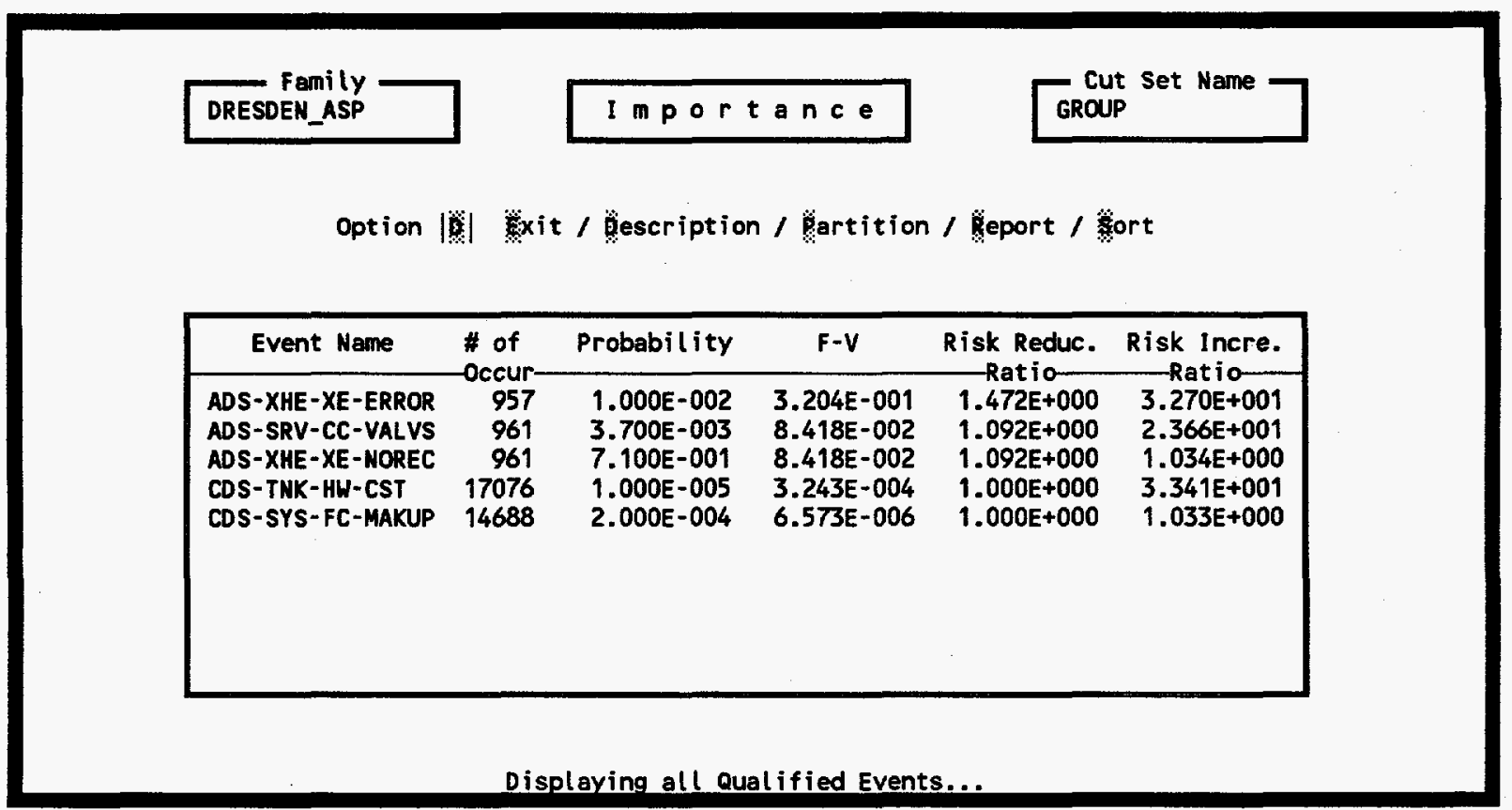

Figure 21. Example of the Importance screen using the Complement option.

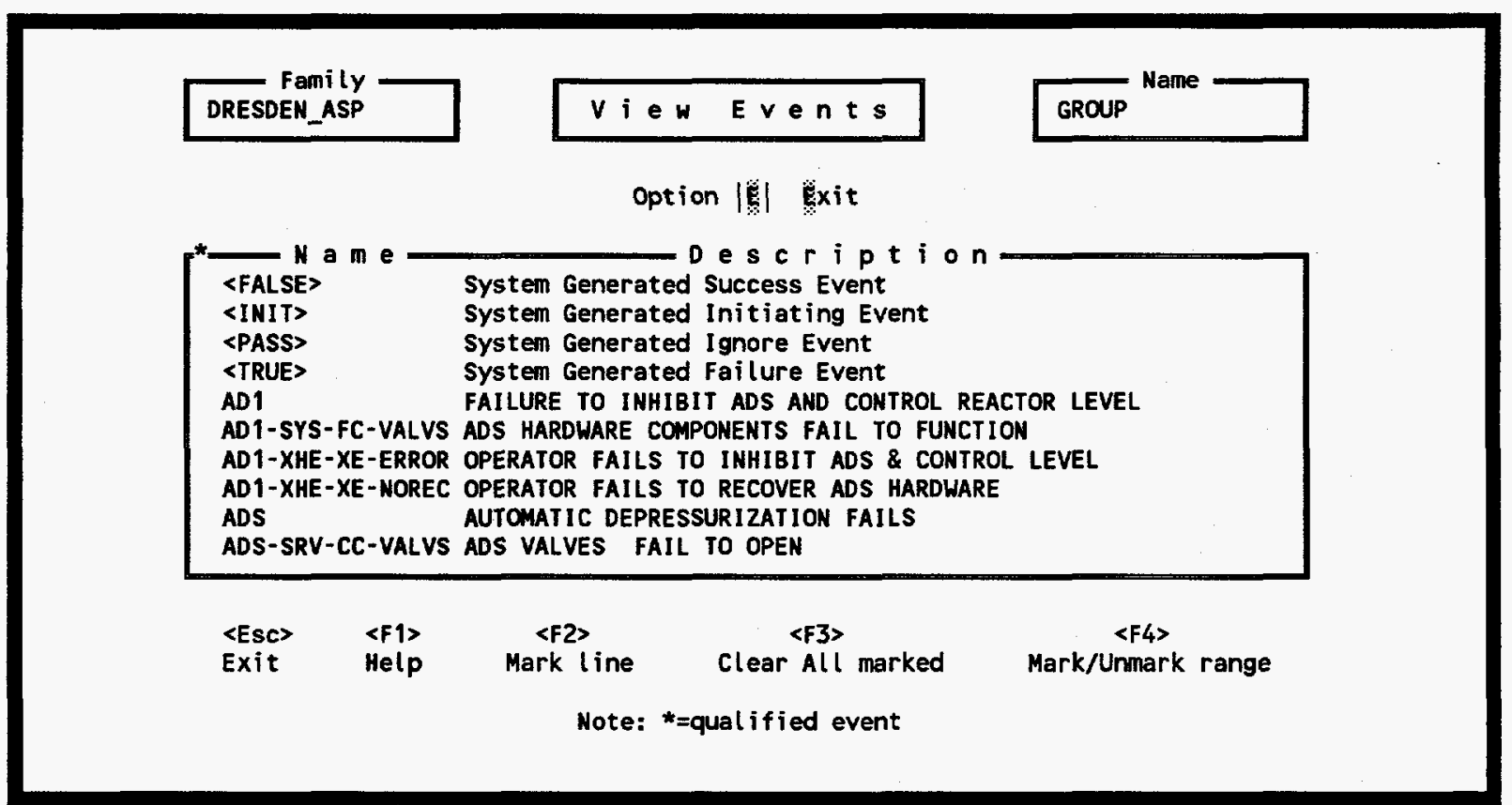

Figure 22. View Events screen.

c. Mark the qualified events using $\langle F 2\rangle,\langle F 3\rangle$, or $\langle F 4\rangle$. See Section 1.9.2 for more details on the marking keys. 
Initiating Event Assessment

d. Press $<$ Enter $>$ to return to the Partition screen.

e. Fill the desired entry fields for removing the events from the list of qualified events.

f. Return to the Importance screen where the number of qualified events will be updated accordingly. If the system cut sets do not contain any of the qualified events, then the screen will display the message, "No cut sets qualify."

3.1.4.5.4 Report-This option generates the Importance Measures Report that consists of the event name, number of occurrences, probability of failure, F-V importance, risk reduction ratio, and risk increase ratio. When you invoke this option, the screen will display the Reports screen (Figure 12). See Section 3.1.4.2 for more details.

3.1.4.5.5 Sort-This option selects the sort order for displaying the events on the screen and printing the events in the Importance Measures Report. Invoke this option as follows.

1. Type $\langle S\rangle$ in the option fields to access the Sort options for the list of events (Figure 23).

As shown, the following sort options are available:

$\begin{array}{lll}\text { Name } & - & \text { Sorted by event name } \\ \text { Occurrence } & - & \text { Sorted by the number of occurrences (most to fewest) } \\ \text { Probability } & - & \text { Sorted by the probability failures } \\ \text { F-V } & - & \text { Sorted in Fussell-Vesely or Birnbaum order } \\ \text { Reduction } & - & \text { Sorted in risk reduction ratio order } \\ \text { Increase } & - & \text { Sorted in risk increase ratio order }\end{array}$

2. Type the highlighted letter associated with the desired sort method to sort the list.

3.1.3.6 Sort. Sort from the Event Assessment screen lets you arrange the sequences or CCDP. Resort the event list as follows.

1. Type $\langle S\rangle$ in the option field.

2. Press $<$ Enter $>$ to access the Select Sequence Sort window (Figure 24).

3. Highlight either Name to sort by sequence Conditional Core Damage Prob to sort by CCDP using the up and down arrow keys.

4. Press < Enter $>$ to resort the list and return to the Event Assessment screen. 


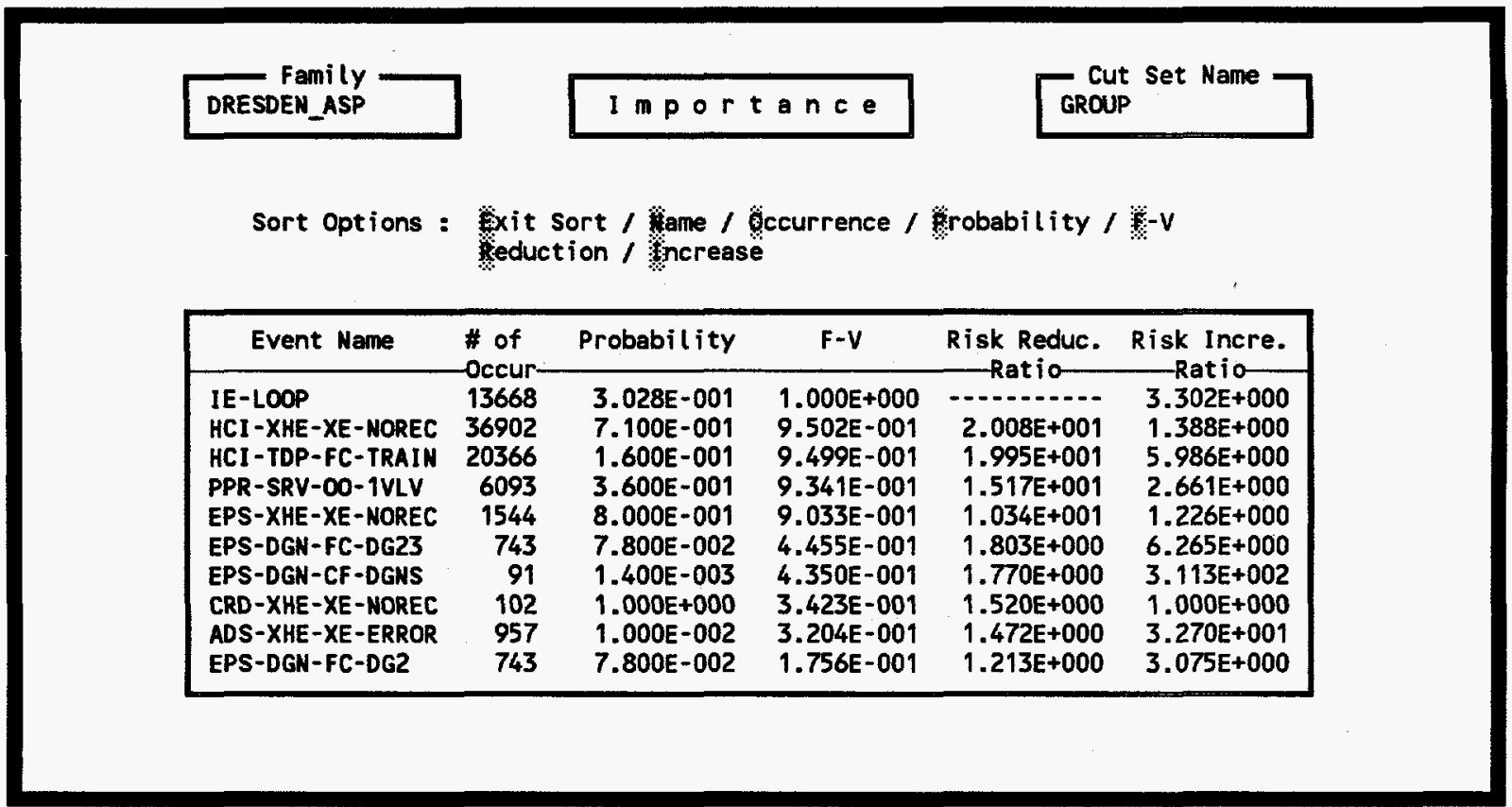

Figure 23. Sort options for the list of events.

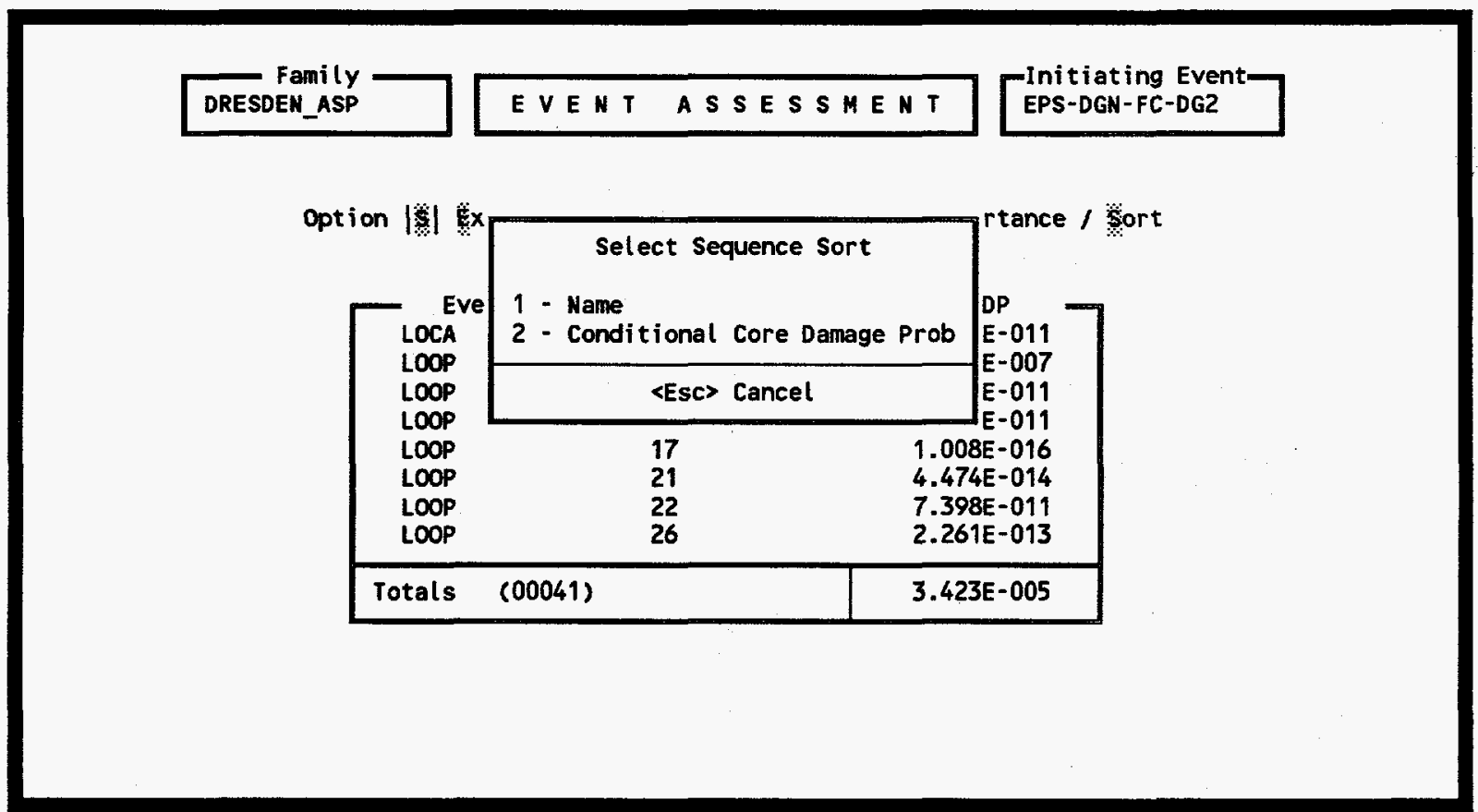

Figure 24. Select Sequence Sort window. 
Initiating Event Assessment

\subsection{Retrieving an Initiating Event Assessment}

Use the Retrieve option to retrieve an initiating event assessment. This option lets you modify, copy, delete, and rename a resident initiating event assessment.

1. Highlight an initiating event assessment by using the up and down arrow keys, or use the speed search feature to locate an initiating event. See Section 1.8.1 for more details about speed search.

2. Press $\langle F 2\rangle$ to retrieve the initiating event assessment and access the Assessments screen (Figure 25).

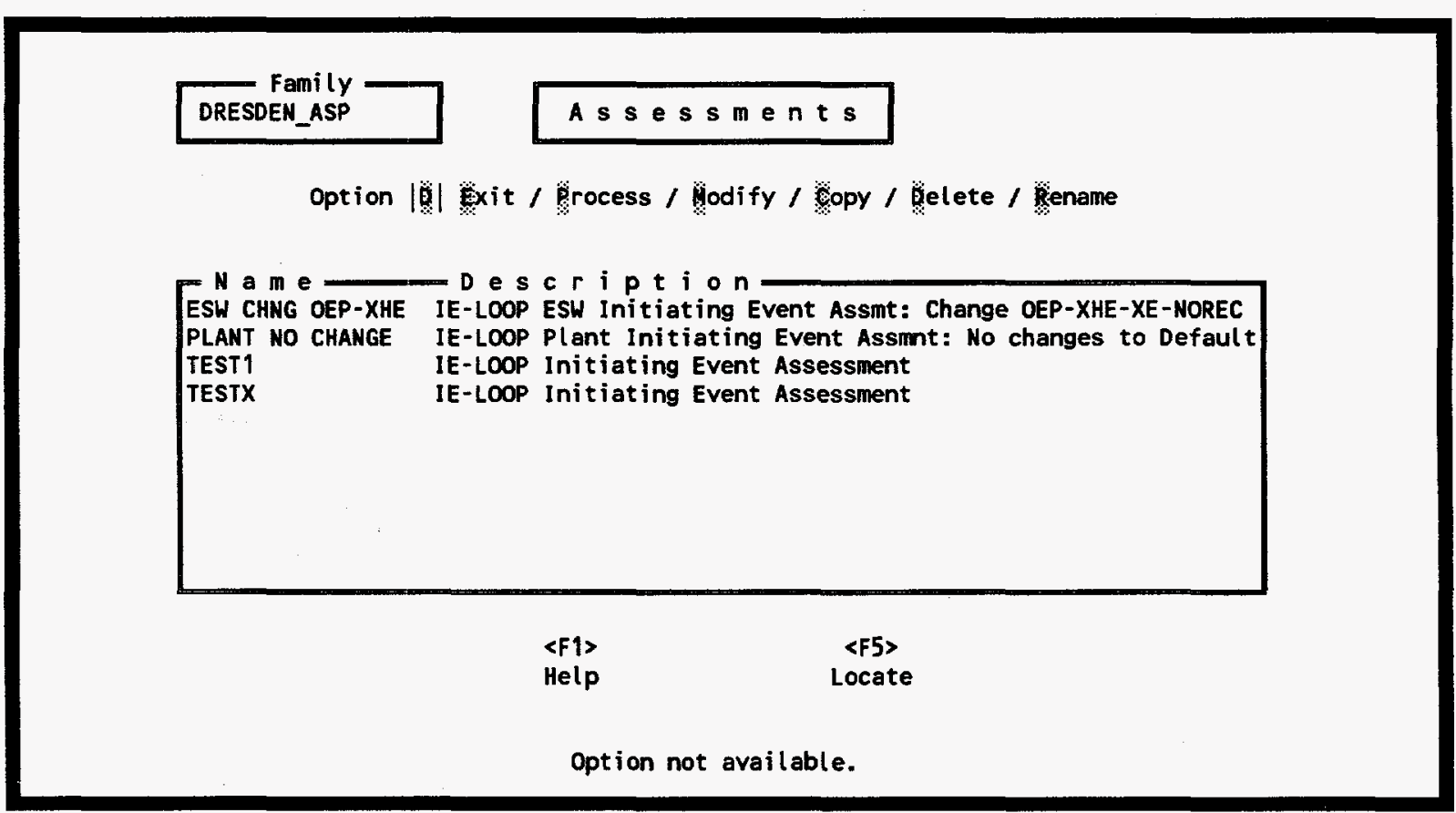

Figure 25. Assessments screen.

At this screen, you can process the assessment; modify the assessment before processing it; and copy, delete, or rename the assessment.

\subsubsection{Process}

Process regenerates the event data as well as loads and solves the logic for the associated accident sequences and requantifies the cut sets. After highlighting the event assessment, perform the following procedure.

1. Type $<\mathrm{P}>$ in the option field. 
2. Press <Enter $>$ to generate the new event data and to access the Event Assessment screen (Figure 11).

3. See Section 3.1.4 for more information regarding the Event Assessment screen.

\subsubsection{Modify}

Modify Event gives you the option to change current-case values for the events listed on the screen.

1. Highlight an event using the up and down arrow keys.

2. Type $\langle M\rangle$ in the option field.

3. Press <Enter $>$ to access the Modify Data screen (Figure 9).

4. See Section 3.1.4 for more information regarding the Modify Data screen.

5. Type $<\mathrm{E}>$ in the option field and press $<$ Enter $>$ to return to the Initiating Events screen.

\subsubsection{Copy}

Copy allows you to copy and rename an existing event ID for analysis.

1. Type $\langle\mathrm{C}\rangle$ in the option field.

2. Highlight the desired event ID using the up and down arrow keys.

3. Press <Enter $>$ to access the Copy Event ID screen (Figure 26). If you do not rename the event ID, the screen will display the message, "Event ID exists, overwrite? (Y/N)"

a. Type $\langle\mathrm{Y}\rangle$ to overwrite the event ID or $\langle\mathrm{N}\rangle$ to enter another event ID name.

4. Type $<\mathrm{C}>$ to copy the highlighted event ID into the new event ID and add it to the list of event IDs. Type $\langle E\rangle$ to quit the copy option without copying the event ID and return to the Assessments screen.

\subsubsection{Delete} follows.

This function is used to remove an event ID from the list of event IDs. Invoke this option as

1. Type a $\langle D>$ in the option field.

2. Highlight the event ID you want to delete from the list of event IDs. 
Initiating Event Assessment

$$
\text { Copy Event I D }
$$

Option | $\mid$ Exit / êpy

\begin{tabular}{|llll}
\hline $\begin{array}{l}\text { Name } \\
\text { Description }\end{array}$ & TEST11 & Date & 1994/12/14 \\
\hline
\end{tabular}

Figure 26. Copy Event ID screen.

3. Press < Enter > to access the Delete Event ID screen (Figure 27).

Delete Event I D

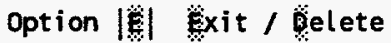

\begin{tabular}{llll}
\hline $\begin{array}{l}\text { Name } \\
\text { Description }\end{array}$ & TEST1 & Date & 1994/12/14 \\
\hline
\end{tabular}

Figure 27. Delete Event ID screen.

4. Type $<D>$ and press $<$ Enter $>$ to delete the selected event ID and return to the Assessments screen. Type $\langle E\rangle$ to quit the Delete option without deleting the event ID and return to the Assessments screen. 


\subsubsection{Rename}

Rename lets you change the current event ID name and associated description. This option also allows you to edit the text associated with the event ID.

1. Type an $\langle R\rangle$ in the option field.

2. Highlight the event ID you want to edit in the list of event IDs.

3. Press < Enter $>$ to access the Rename Event ID screen (Figure 28).

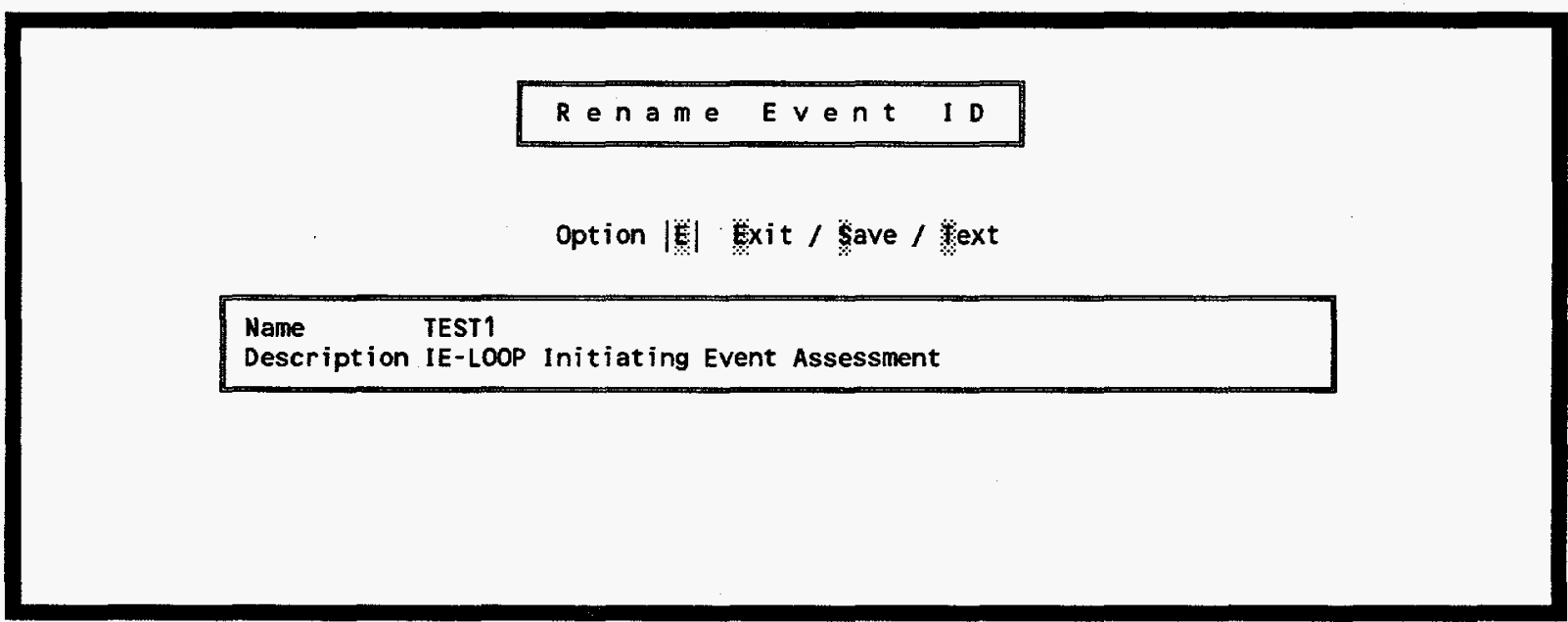

Figure 28. Rename Event ID screen.

4. (Optional) Place the cursor in the Name or Description fields and enter the new name or description by typing over the old information.

5. (Optional) Type $<\mathrm{T}>$ and press $<$ Enter $>$ to edit the current text or attach text to the renamed event ID.

6. Type $<S>$ and press $<$ Enter $>$ to save the renamed event ID and return to the Assessments screen.

\subsection{Event Probability Changes Screen}

In the Event Probability Changes screen, you can change the current-case values for a single event or group of events without changing the base-case values. This screen allows you to change values for the Random Failure Data fields, Uncertainty fields, and the Process Flag field. Figure 29 and Figure 30 show the Event Probability Changes screen for a single event and a group of events, respectively. Enter current-case values as follows. 


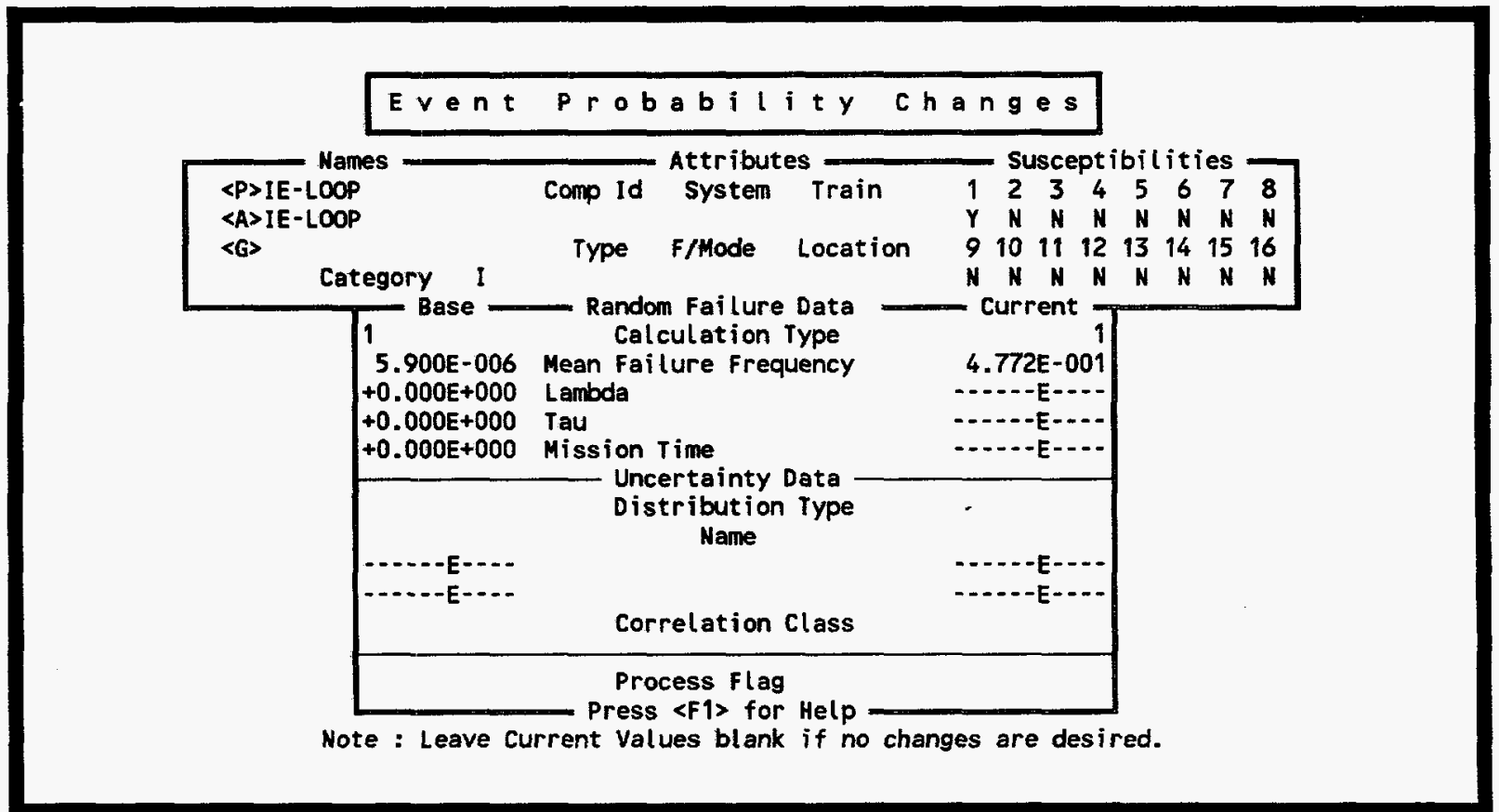

Figure 29. Example of the Event Probability Changes screen for a single event case.

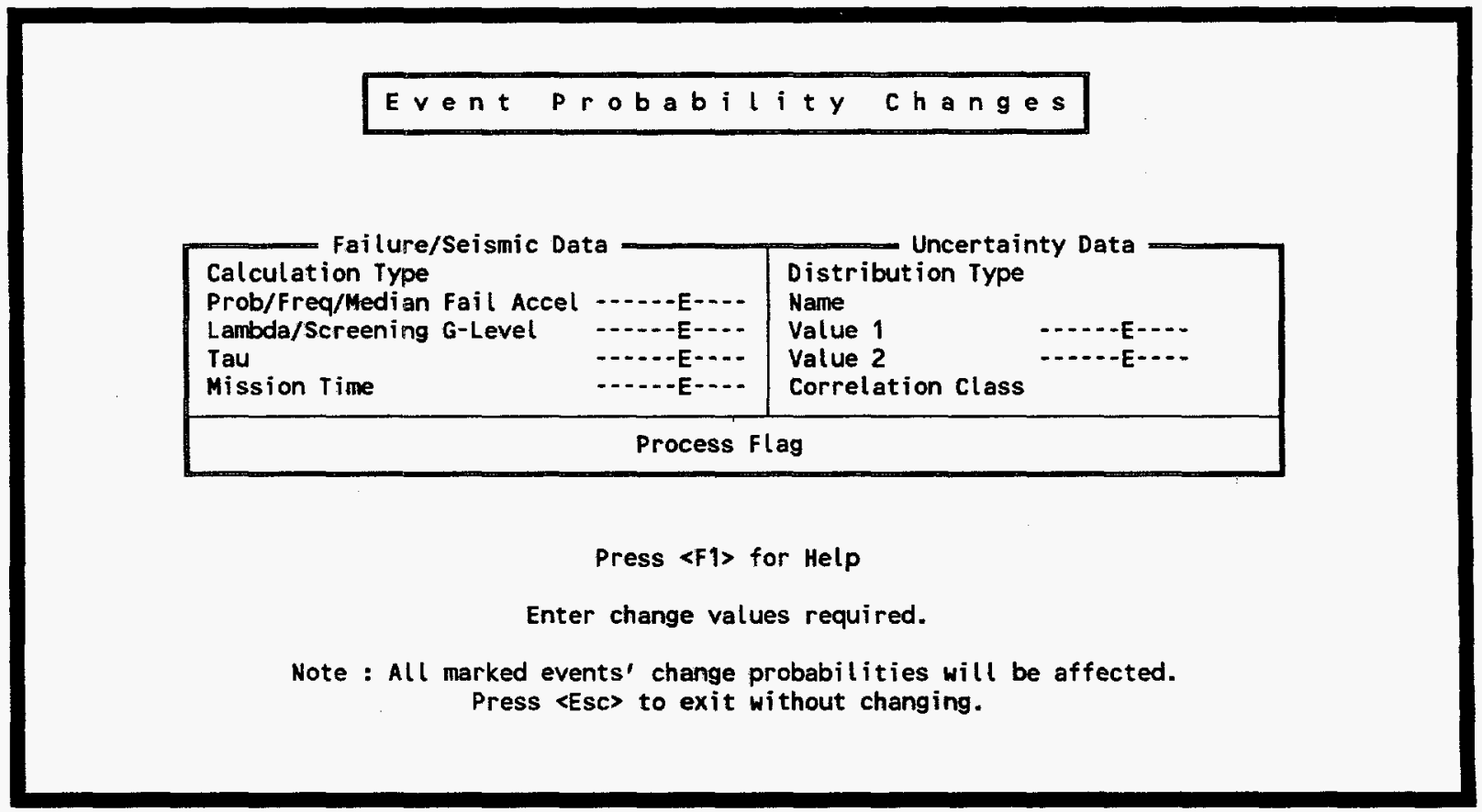

Figure 30. Example of the Event Probability Changes screen for the group of events case. 
1. Tab to the current-value field you want to change.

2. Enter the new value into the field.

a. Press $<$ F1 $>$ to access online help, if you need an explanation of the field. See Section 3.3.1 for more information regarding the online help.

b. Select a value from the online help or exit the help screen.

3. Change the remaining fields for analysis by repeating Steps 1 and 2 .

4. Press $<$ Enter $>$ to save the changes to the database and return to the Modify Data screen. Press $\langle$ Esc $\rangle$ to abort the changes and exit the screen without saving the changes.

\subsubsection{Event Probability Changes Screen Online Help}

The online help consists of three main screens. The first screen consists of failure data calculation types with an explanation associated with each calculation type. The second screen contains uncertainty distribution types and the equation used for each distribution type. The third screen provides the special processing options.

3.3.1.1 Failure Data Calculation Types. In the Failure Data box, the calculation type consists of an alpha numeric reference to the calculation method you can use. Seventeen calculation types, designated 1 through 9, T, F, I, S, G, L, M, and H, are available. Enter a calculation type as follows.

1. Position the cursor in the Calculation Type field.

2. Type the desired calculation type designator, or press $\langle F 1\rangle$ for an explanation of these calculations. Figure 31 shows the help screen. Highlight the calculation type and press $<$ Enter > to exit the help screen.

3.3.1.2 Uncertainty Distribution Types. For the Uncertainty Data box, nine predefined distribution types are available. The predefined distribution types are normal, log-normal, beta, gamma, chi-squared, exponential, uniform, maximum entropy, and seismic. Besides these predefined distribution types, you may use user-defined histograms. The default distribution type is no distribution. Figure 32 shows the help form associated with the distribution type field on the Event Probability Changes screen. From this help screen, help forms associated with each nine predefined distribution types are available. View these distribution help forms as follows.

1. Position the cursor on the desired distribution type.

2. Press the $\langle$ F1 $\rangle$ key.

3. Highlight Distribution Type and press < Enter $>$ to exit the help screen. 


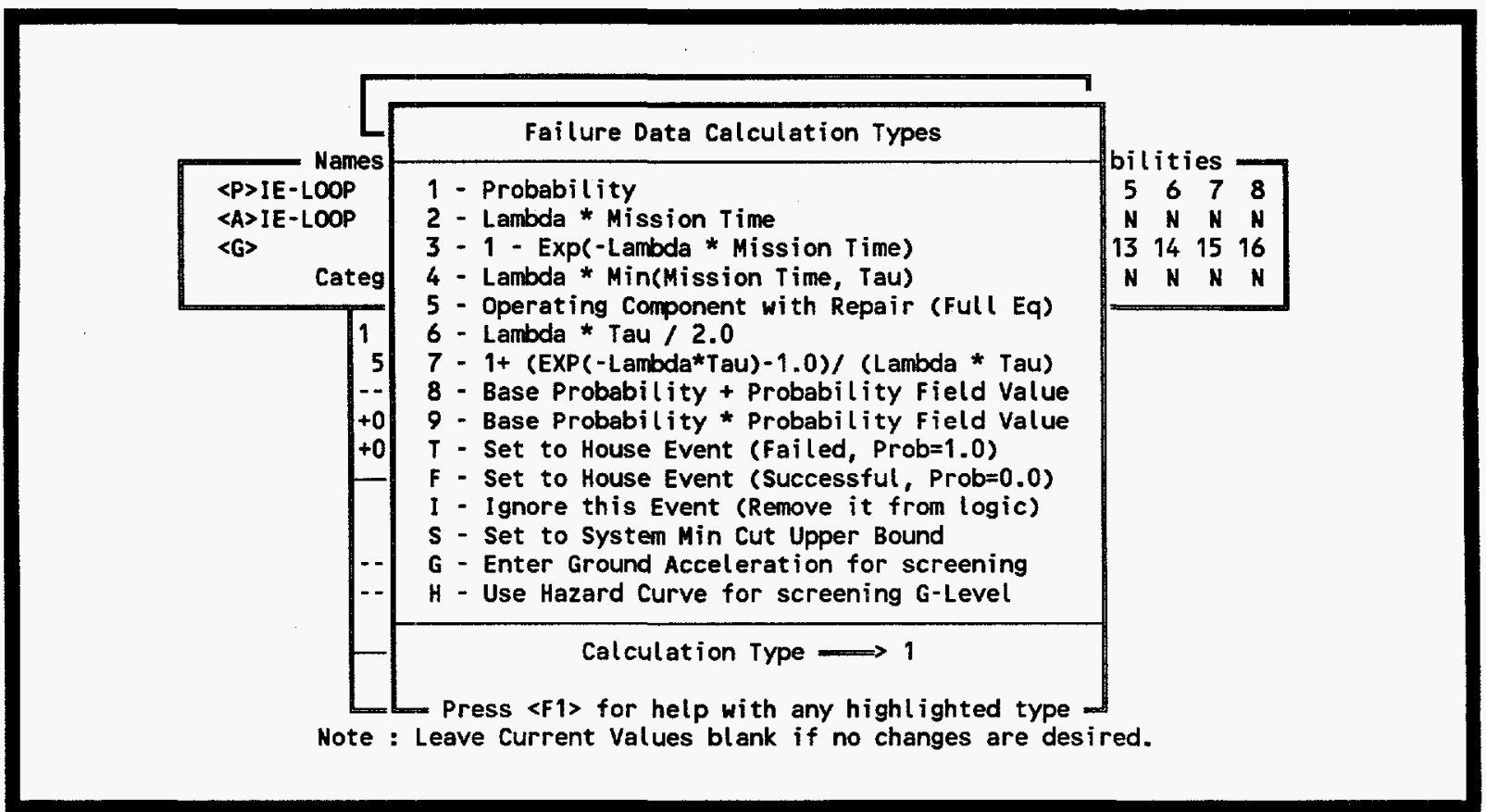

Figure 31. Failure Data Calculations Types online help screen.

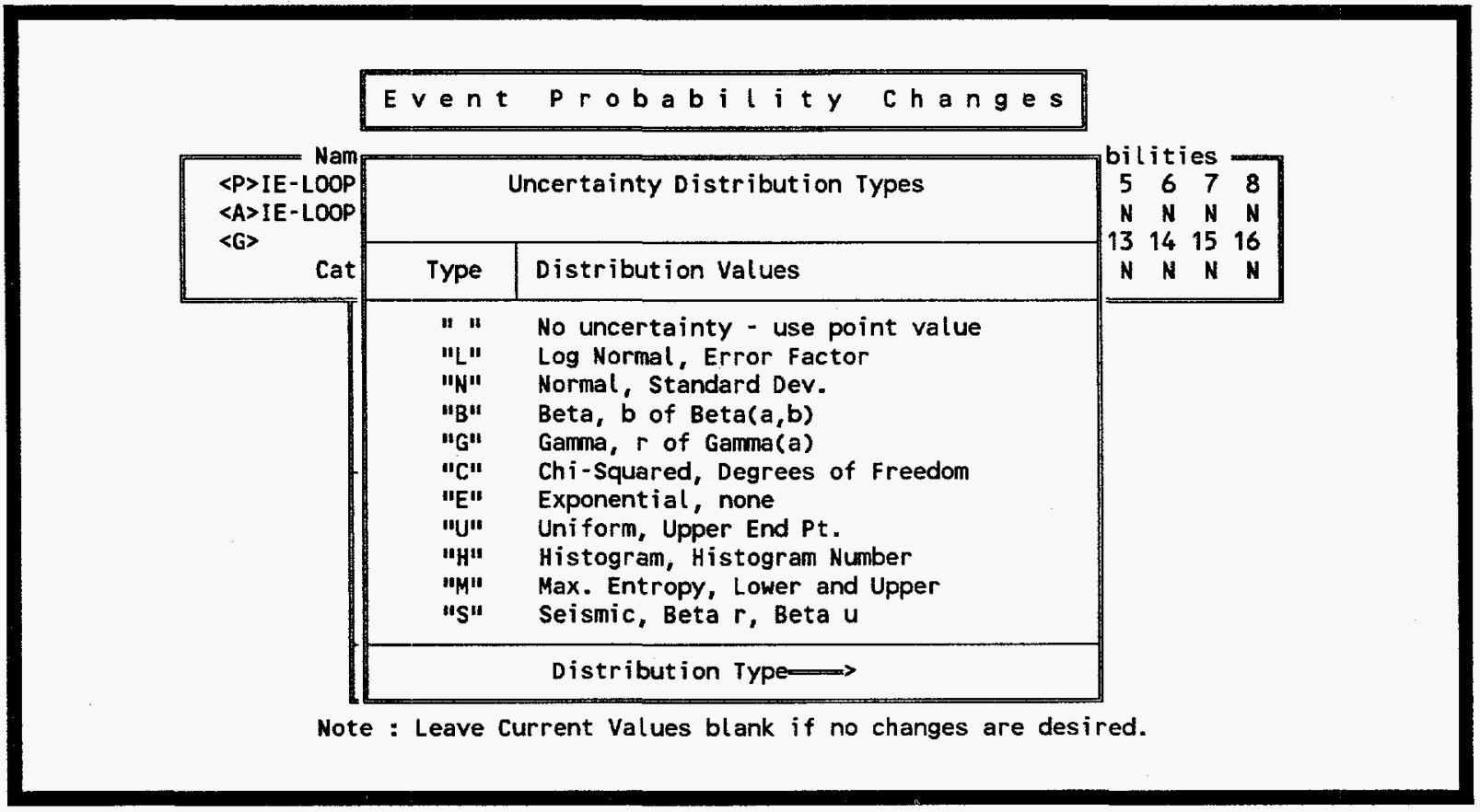

Figure 32. Uncertainty Distribution Types online help screen. 
GEM uses correlation to account for data dependencies among like basic events in the database. Correlation classes consist of four-character, upper-case values. A blank correlation class indicates that no data dependencies exist. When running the uncertainty analyses, all basic events in the same correlation class will use the same sample value.

3.3.1.2 Process Flag. The Miscellaneous area contains the process flag, which is a onecharacter field that specifies if certain processes should take special note of the selected event. Figure 33 shows the help screen for the Process Flag field. You can input one of the following upper-case values.

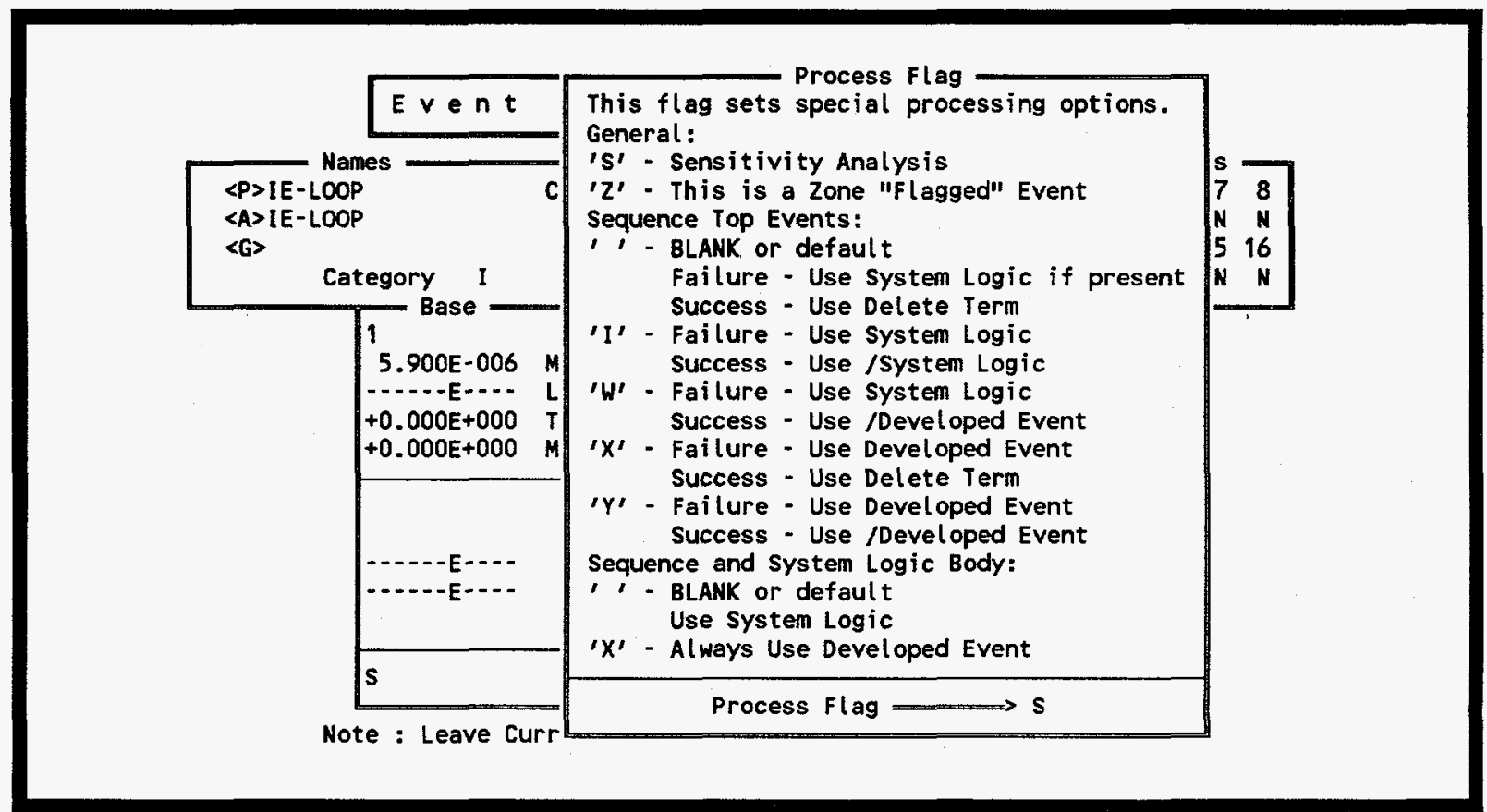

Figure 33. Process Flag online help screen.

For a general event,

'S' - sensitivity analysis. For events marked for sensitivity analysis, GEM will map a core damage frequency plot. A sensitivity analysis allows you to see how sensitive the core damage frequency is in relation to an event.

' $Z$ ' - zoned flagged event. A zone flagged event is an event that has been marked as representing a zone (i.e., location or area). An example of a zone is a fire zone or a flood zone. When GEM encounters a zone flagged event, it performs a transformation. A transformation is an event or set of events that replace a zone flagged event. 


\section{Initiating Event Assessment}

For a sequence top event,

'I' - failure. Use system logic (if top event fails), or use the complement of the system logic (if top event succeeds). That is, if the top event is a failure, GEM will expand the fault tree and solve; if the event succeeds, GEM will complement the fault tree logic and solve it. An "I" causes GEM to treat the transfer as independent. Logic below this transfer is expanded for failure references, and for success references the complement of the logic is used.

'W' - failure. Use system logic (if top event fails), or use complement of the developed event (if top event succeeds). That is, if the event fails, GEM will expand the fault tree and solve; if the event succeeds, GEM will use the complement of the developed event for the system.

'X' - failure. Use developed event (if event fails), or use cut set matching to eliminate cut sets (if event succeeds). That is, an " $\mathrm{X}$ " tells GEM that the basic event is to be used for failure references, but success references are to be treated the same as if the flag was blank.

'Y' - failure. Use developed event (if event fails), or use complement of developed event (if event succeeds). That is, a "Y" indicates that a transfer is to be replaced with its basic event for failed references and the complement of the event is to be used for success references.

For sequence and system logic,

' $\mathrm{X}$ ' - always use developed event. That is, never expand the fault tree and solve; always use the event.

When the Process Flag field is blank, the transfer associated with this event is expanded for failure references. For success references, the transfer is also expanded; however, the cut sets generated are removed from the failure cut sets using cut set matching. 


\section{CONDITION ASSESSMENT}

A condition assessment evaluates an operational occurrence in which no initiating event has taken place, but equipment failures, unavailabilities, or degradations have reduced the reliability of one or more systems to operate under off-normal conditions. The condition assessment option allows one to analyze working models based on the duration of the event. The calculated results provide information pertaining to the CCDP. Refer to Section B-2.2.3 for a technical discussion of this topic.

1. Name Event ID - The Event ID equates to a unique identifier assigned to the particular operational event undergoing analysis. By using this identifier, you can readily return to a previously completed analysis or to one that is in progress without losing any information.

2. Designating the Event Duration - This step provides GEM the number of hours that the condition being analyzed existed.

3. Modify Data - This step allows you to stipulate the impact from the event analysis to the basic events of the model and to determine the revised basic event values.

4. Process - This step takes the inputs that you just established and processes the SAPHIRE model to calculate the CCDPs for each of the appropriate accident sequences and the overall model. GEM generates the new basic event data, regenerates and requantifies the sequence minimal cut sets, and displays the results associated with the impacted sequences.

5. Results Display and Post-processing - You can sort the results and generate various reports, which are compatible with the Accident Sequence Precursor program analysis methods. You can also list and partition the new sequence minimal cut sets as well as calculate and display the importance measures.

\subsection{Performing a Condition Assessment}

Initiate the Condition Assessment option as follows:

1. Type $<\mathrm{C}>$ or highlight Condition Assessment using the up and down arrow keys.

2. Press < Enter $>$ to access the Name Event ID screen (Figure 34).

\subsubsection{Name Event ID}

The Name Event ID screen allows you to assign a unique name, enter the date, and attach a description to the event analysis (Figure 34).

You can create or retrieve a condition assessment. If you create a new assessment, you can add, modify, or reset the basic events before processing the assessment. If you retrieve an existing condition assessment, you can modify, copy, delete, or rename the assessment. 


\section{Condition Assessment}

NOTE: Although Section 3 describes the options available for initiating event assessments, most options in Section 4 function in the same manner for condition assessments. Therefore, this section references Section 3 whenever possible.

$$
\text { Name Event I D }
$$

Option |S| Exit / Save / Mext Edit / Retrieve Assessment

\begin{tabular}{lll}
\hline $\begin{array}{l}\text { Name } \\
\text { Description Condition Assessment }\end{array}$ Date $1995 / 01 / 16$ \\
\hline
\end{tabular}

Figure 34. Name Event ID screen.

1. Assign a name to the event ID, using the Name field. If the Name field is left blank, the message "Enter an Event ID name" will appear at the bottom of the screen. To continue, enter a name for the event assessment.

2. (Optional) Tab to the Date field. You can change the default date by typing over the date. The date must remain in the same format presented in the field.

3. (Optional) Tab to the Description field. Enter a brief description of the event analysis. If you require more than the 60 -character event description provided in the Description field, you can attach an additional event description by typing a $\langle\mathrm{T}\rangle$ into the option field and pressing $<$ Enter $>$.

4. Place the cursor in the option field using the arrow keys.

5. Type $\langle S\rangle$ into the Option field.

6. Press <Enter $>$ to save the event ID and access the Condition Assessment screen (Figure 35). If a duplicate record exists in the database, a message at the bottom of the screen will ask if you want to overwrite the record. Type $\langle\mathrm{Y}\rangle$ to overwrite the record or $\langle\mathrm{N}\rangle$ to rename the event analysis.

\subsubsection{Designate the Event Duration}

Event duration is the period of time the condition existed. Event duration allows you to calculate the CCDP for the duration of a condition.

1. Enter the event duration in hours in the Condition Assessment screen field (Figure 35). 


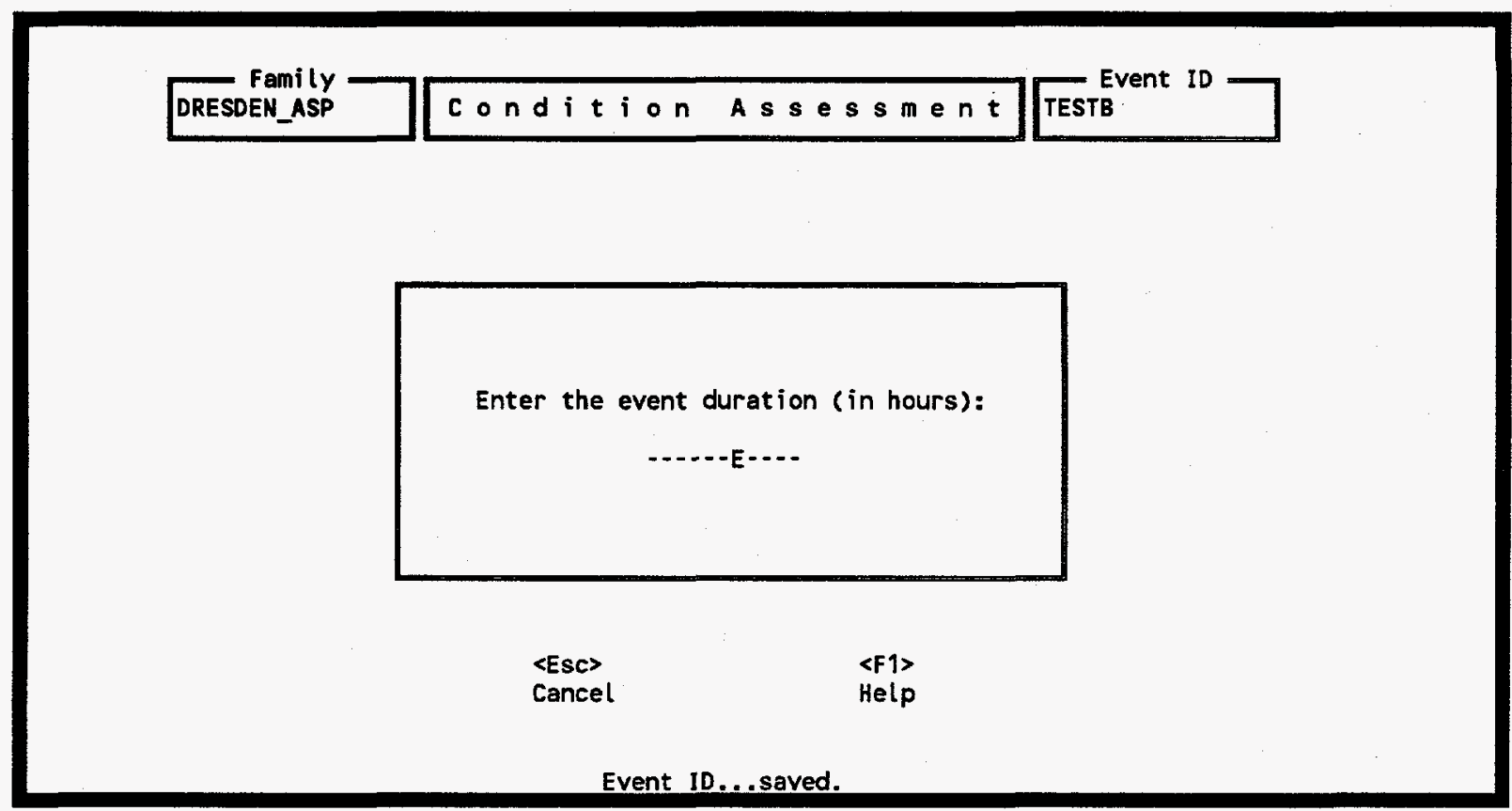

Figure 35. Condition Assessment screen.

2. Press $<$ Enter $>$ to access the Modify Data screen.

\subsubsection{Modify Data}

Modify Data allows you to make changes to the existing list of events, if any, add events to the list, change event values, copy an existing data set into the current data set, or reset an event to its basecase value. Process generates current-case event data, and generates and quantifies new accident sequence minimal cut sets. GEM creates reports that reflect the event modifications that currently exist within the database. Modify Data in the Condition Assessment functions in the same manner as Section 3.1.3 in the Initiating Event. Modify Data differs in the condition assessments from the initiating events with the Modify Event option.

Initially, Modify Data display a screen void of any events unless you have retrieved a previous assessment. See Section 3.1.3 for more details on the options. See Appendix B for an example of a condition assessment report.

\subsubsection{Condition Assessment}

Condition Assessment allows you to generate assessment reports for the event ID, display cut sets, and resort the event tree sequences (Figure 36). Condition Assessment functions in the same manner as Section 3.1.4 in the Initiating Event. The Condition Assessment feature differs from the Event Assessment feature by providing the Duration option and sorting by different parameters in the Sort option. See Section 3.1.4 for further information on the other options. 


\begin{tabular}{|c|c|c|c|c|}
\hline DRESDEN_ASP & \multicolumn{4}{|c|}{ CONDITION ASSESSMENT } \\
\hline Option | & \multicolumn{4}{|c|}{$\begin{array}{l}\text { Exit / Keport / Eutsets / Gogic / Jmportance } \\
\text { Buration / Sort / Uncertainty / Ufifertainty Results }\end{array}$} \\
\hline & \multicolumn{3}{|c|}{ Event Duration (hours): $2.000 E+000$} & \multirow[b]{2}{*}{ Importance } \\
\hline Event Tree & Sequence & CCDP & CDP & \\
\hline $\begin{array}{l}\text { LOCA } \\
\text { LOCA } \\
\text { TRAN } \\
\text { TRAN } \\
\text { TRAN } \\
\text { TRAN } \\
\text { TRAN } \\
\text { TRAN }\end{array}$ & $\begin{array}{l}35 \\
36 \\
05 \\
09 \\
36 \\
37-04 \\
37-34 \\
38-39\end{array}$ & $\begin{array}{l}2.279 E-012 \\
3.413 E-011 \\
1.873 E-012 \\
2.930 E-013 \\
4.449 E-013 \\
5.968 E-014 \\
1.642 E-011 \\
2.155 E-010\end{array}$ & $\begin{array}{l}2.278 E-012 \\
3.412 E-011 \\
1.873 E-012 \\
2.929 E-013 \\
4.448 E-013 \\
5.968 E-014 \\
1.642 E-011 \\
2.153 E-010\end{array}$ & $\begin{array}{r}9.998 E-016 \\
1.000 E-014 \\
+0.000 E+000 \\
9.999 E-017 \\
9.999 E-017 \\
+0.000 E+000 \\
+0.000 E+000 \\
2.000 E-013\end{array}$ \\
\hline Totals $\quad(00012)$ & & $4.672 E-010$ & $4.669 E-010$ & $3.112 E-013$ \\
\hline
\end{tabular}

Figure 36. Condition Assessment screen.

4.1.4.1 Cut sets. The option differs from its initiating event counterpart in Section 3.1.4.3 by allowing you to select between current case cut sets and base case cut sets.

NOTE: Displayed cut sets do not have duration factored into the frequency.

1. Highlight an event tree sequence by using the up and down arrow keys.

2. Type $\langle\mathrm{C}\rangle$ in the option field and press $<$ Enter $>$ to access the Cut Set Type pop-up (Figure 37).

3. Highlight either Current Case Cut Sets or Base Case Cut Sets using the up and down arrow keys.

4. Press <Enter $>$ to access the Cut Sets screen. See Section 3.1.4.3 for further details.

4.1.4.2 Duration. This option allows you to change the event duration of the condition assessment. To change the event duration, use the following procedure.

NOTE: Duration is expressed in hours, which is consistent with the Event Reporting System.

1. Type $\langle D>$ in the option field to access the Condition Assessment screen (Figure 38).

2. Enter the event duration value in hours and press < Enter $>$. CCDP values will be recalculated based on the new duration. 


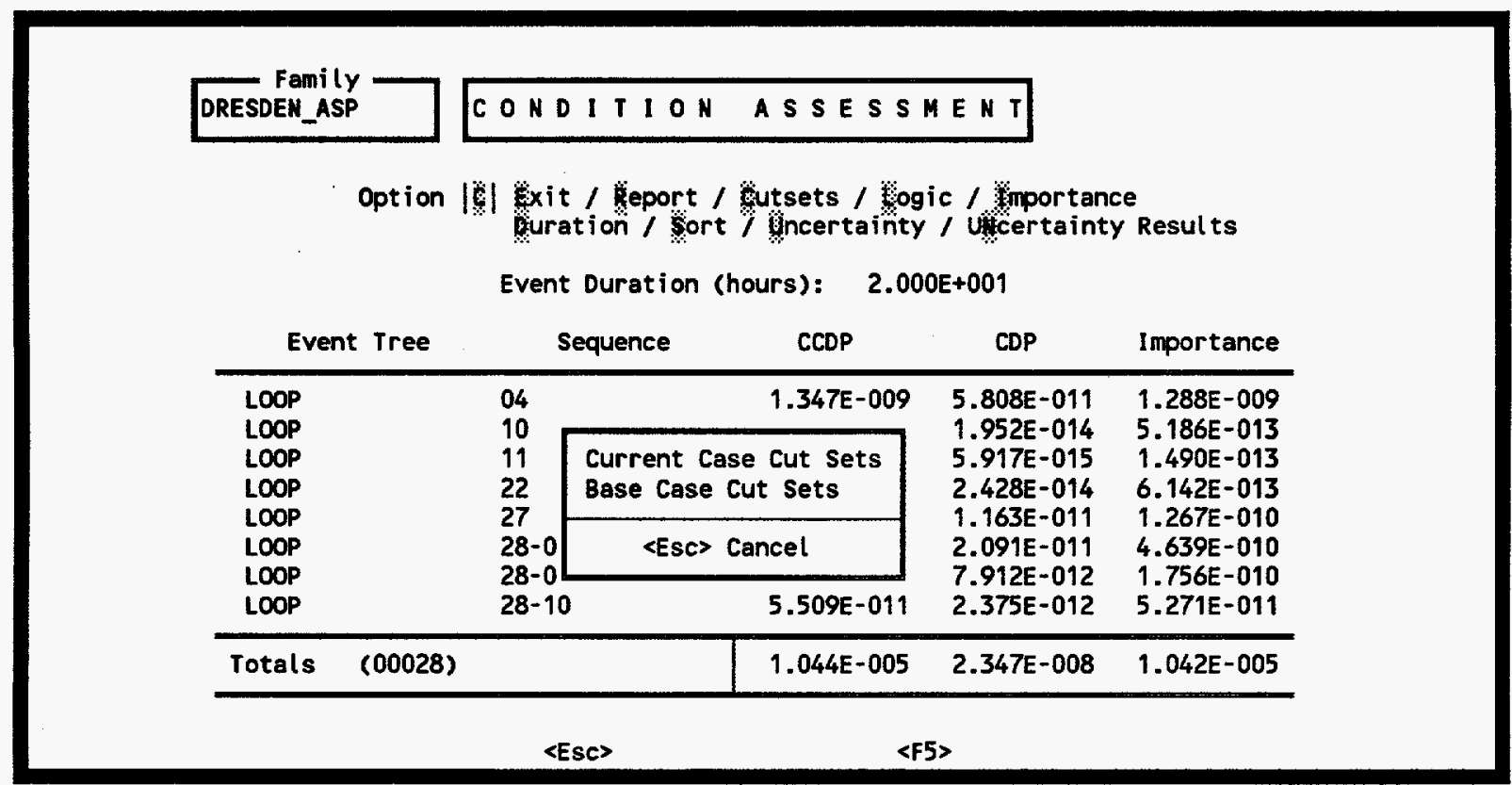

Figure 37. Cut Set Type popup.

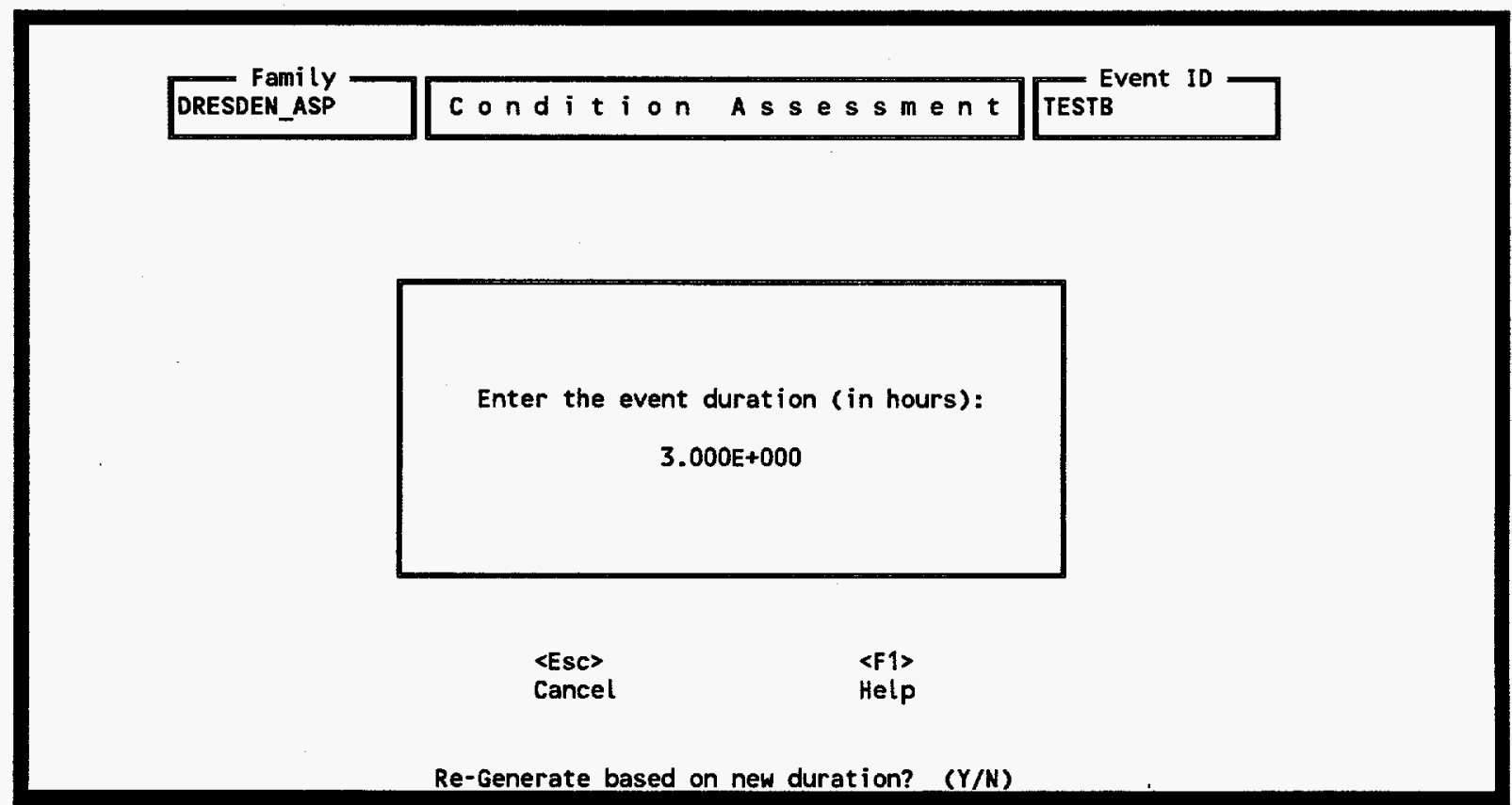

Figure 38. Condition Assessment screen (duration). 


\section{Condition Assessment}

4.1.4.3 Sort. Sort from the Condition Assessment screen lets you arrange the sequences by Name, CCDP, CDP, or importance. Resort the event list as follows.

1. Type $\langle S\rangle$ in the option field.

2. Press <Enter $>$ to access the Select Sequence Sort window (Figure 39).

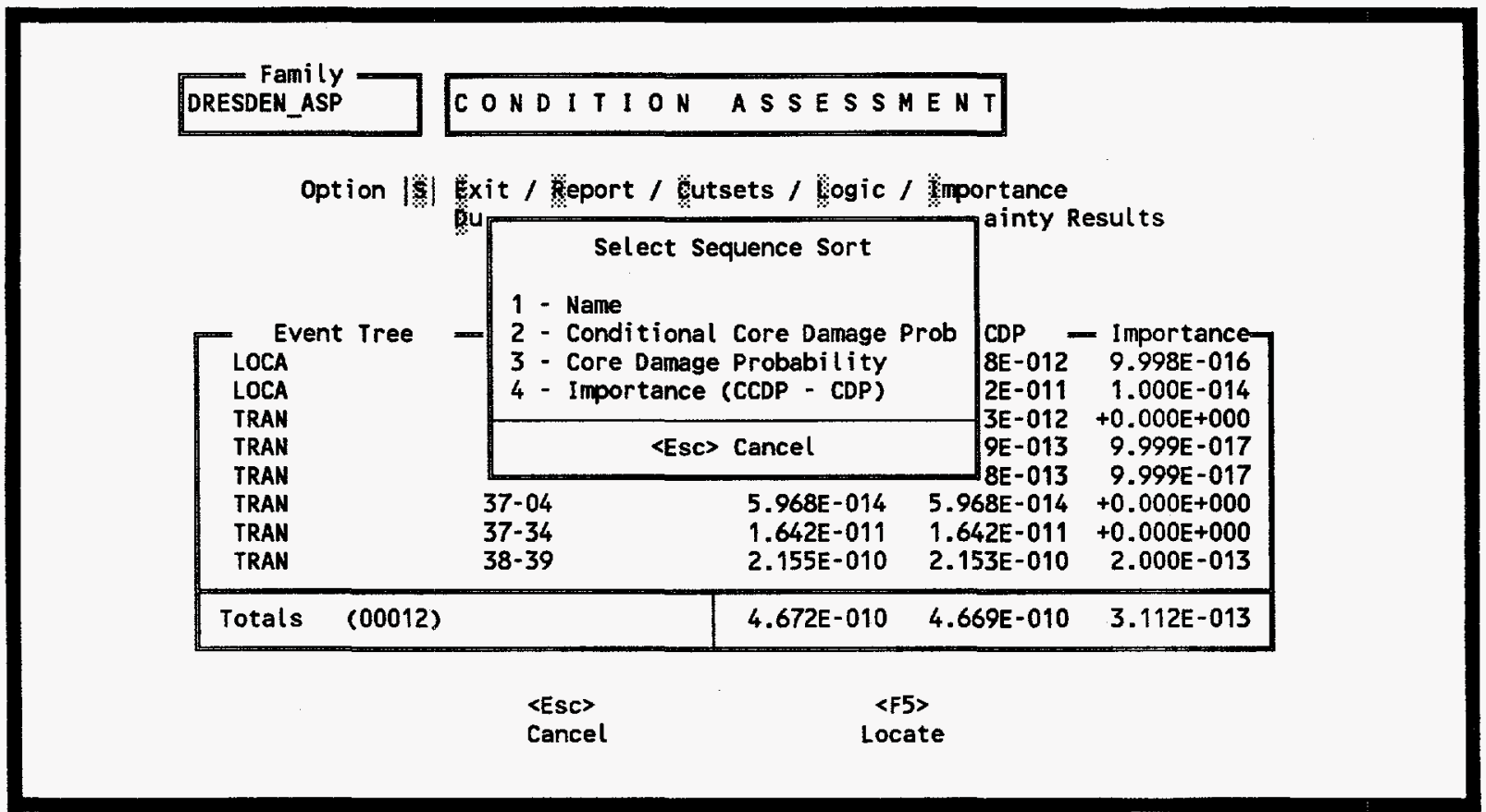

Figure 39. Select Sequence Sort window.

3. Highlight either Name, CCDP, CDP, or importance to sort the sequences by using the up and down arrow keys.

4. Press $<$ Enter $>$ to resort the list and return to the Condition Assessment screen.

4.1.4.4 Uncertainty. The uncertainty analysis allows you to calculate the uncertainty in the top event probability resulting from uncertainties in the basic event probabilities. Access the Uncertainty Calculation Values screen (Figure 40) as follows.

NOTE: $\quad$ The uncertainty of the importance is not yet provided, but it cannot be calculated from the uncertainty of the CDP and of the CCDP.

1. Highlight the desired sequence to analyze.

2. Type $<U>$ in the option field.

3. Press $<$ Enter $>$ to access the Uncertainty Calculation Values screen. 
Figure 40. Uncertainty Calculation Values screen.

4. Type $<\mathrm{L}>$ for Latin Hypercube sampling or $<\mathrm{M}>$ for Monte Carlo sampling in the Sampling Type field.

5. Enter the number of samples to use in the simulation.

6. Enter the seed number for the random number generator if you don't want the clock to set the a random seed.

7. Press <Enter $>$ to process the Uncertainty analysis. While calculating the results, GEM will display interim data in the Processing Family window (Figure 41) before displaying the final results in the Uncertainty Results window (Figure 42).

4.1.4.5 Uncertainty Results. The uncertainty results option allows you to view the Current and Base Quantile Values. Access the Uncertainty Data screen (Figure 43) as follows.

1. Highlight the desired sequence to analyze.

2. Type $\langle\mathrm{N}\rangle$ in the option field.

3. Press $<$ Enter $>$ to access the Uncertainty Data screen.

4. Enter either $\langle\mathrm{C}\rangle$ for current quantile values or $\langle\mathrm{B}\rangle$ for base quantile values. Figure 44 provides an example set of current quantile values. 


\section{Condition Assessment}

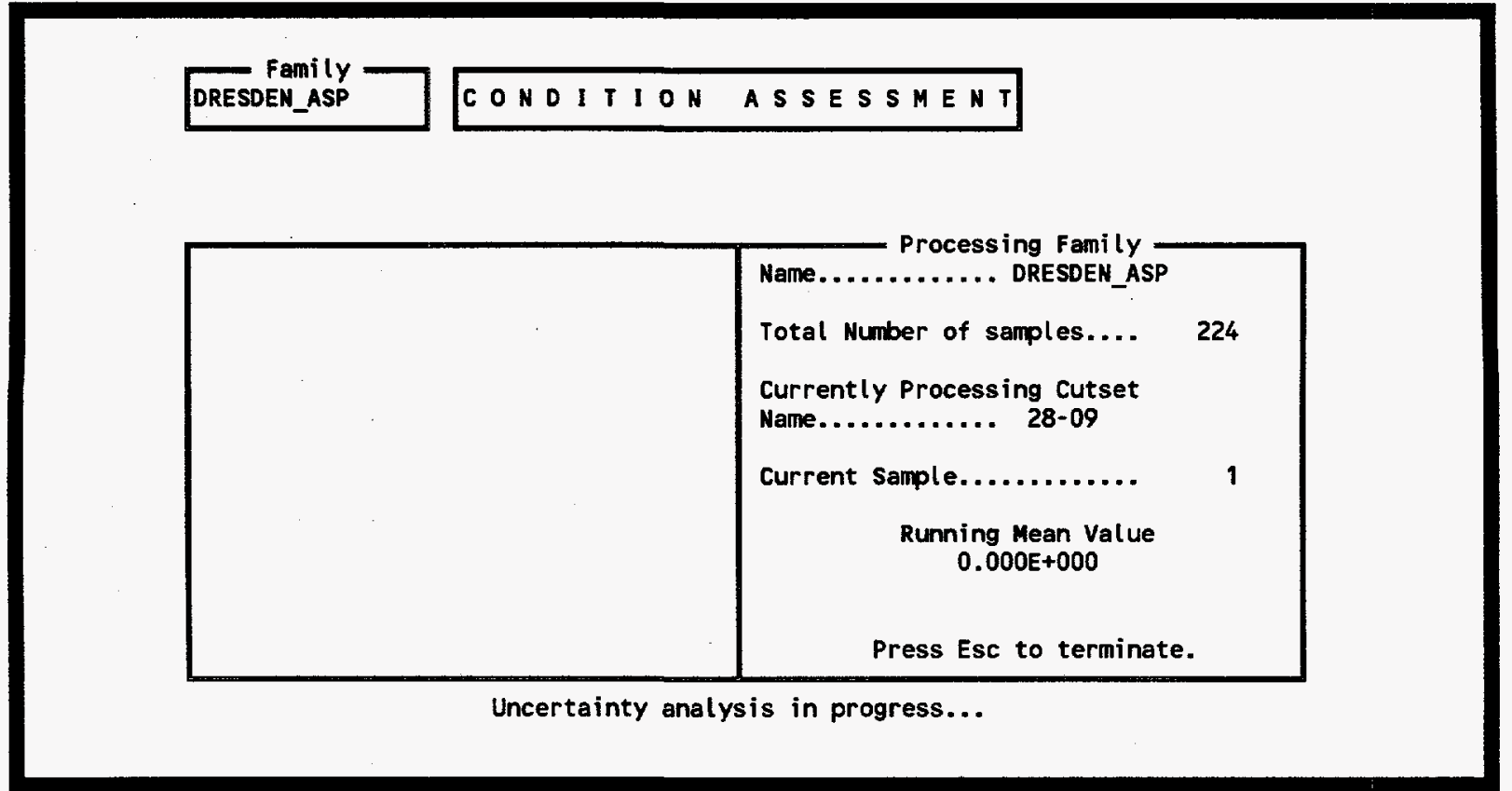

Figure 41. Processing Family window.

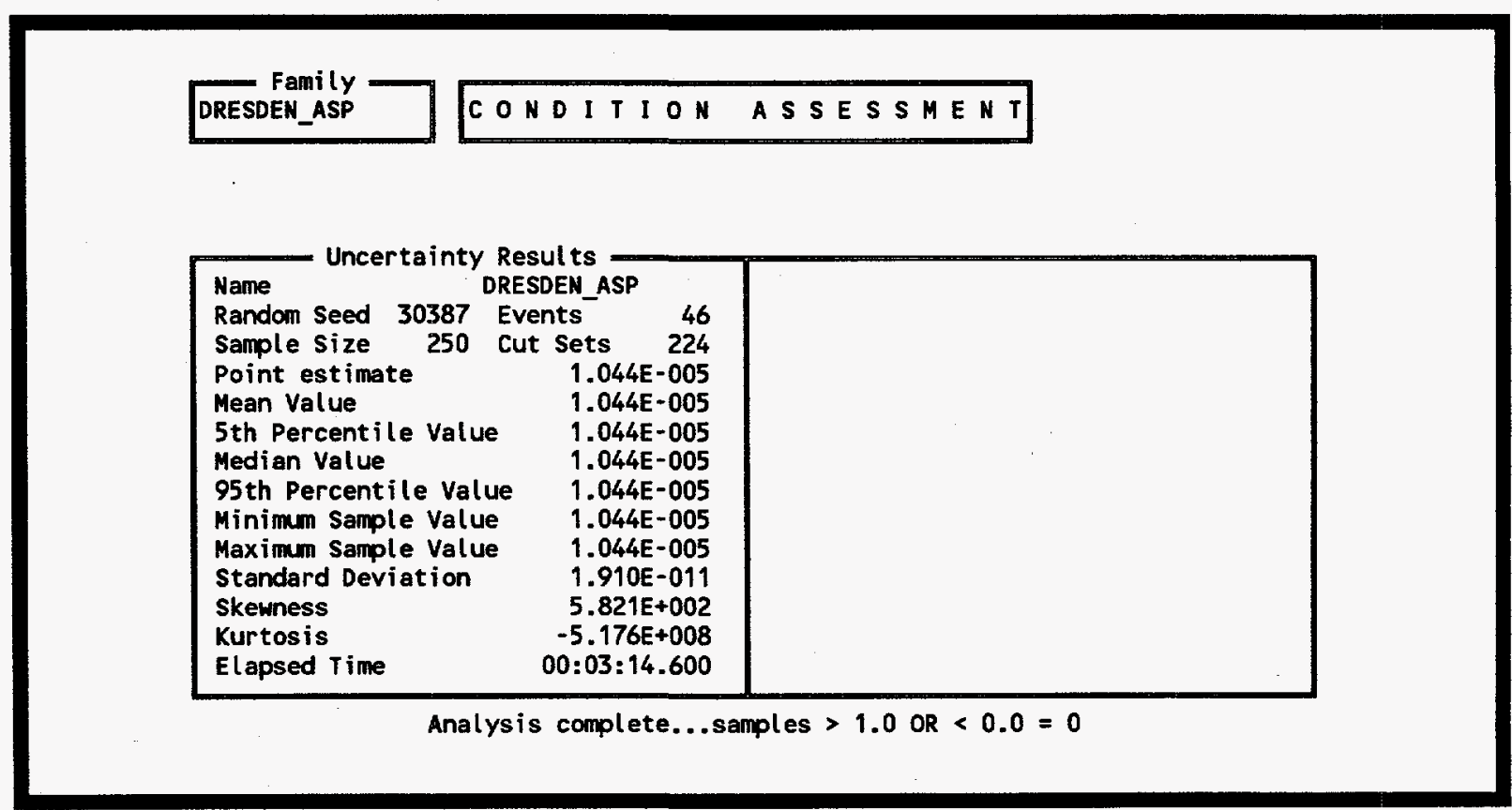

Figure 42. Uncertainty Results window. 


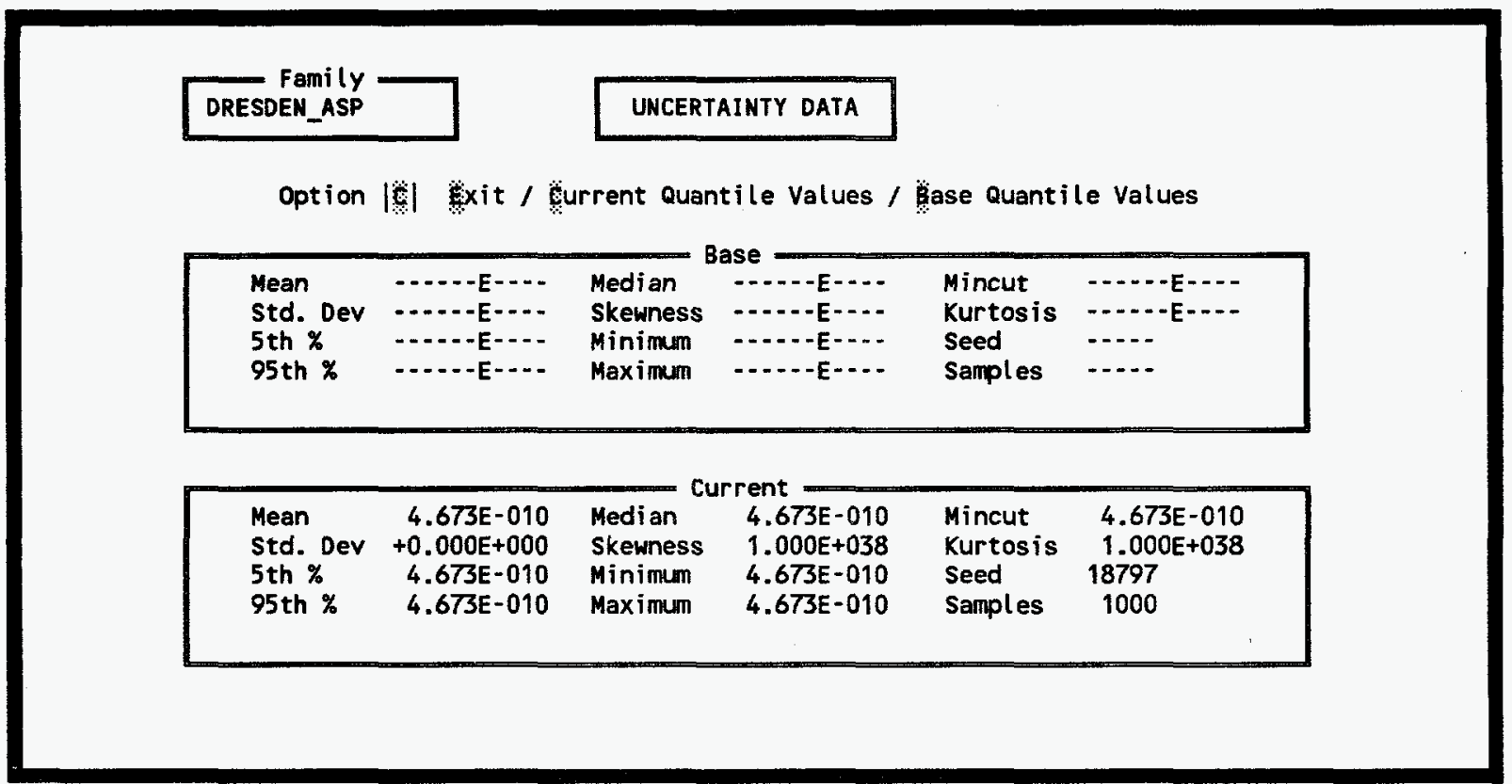

Figure 43. Uncertainty Data screen.

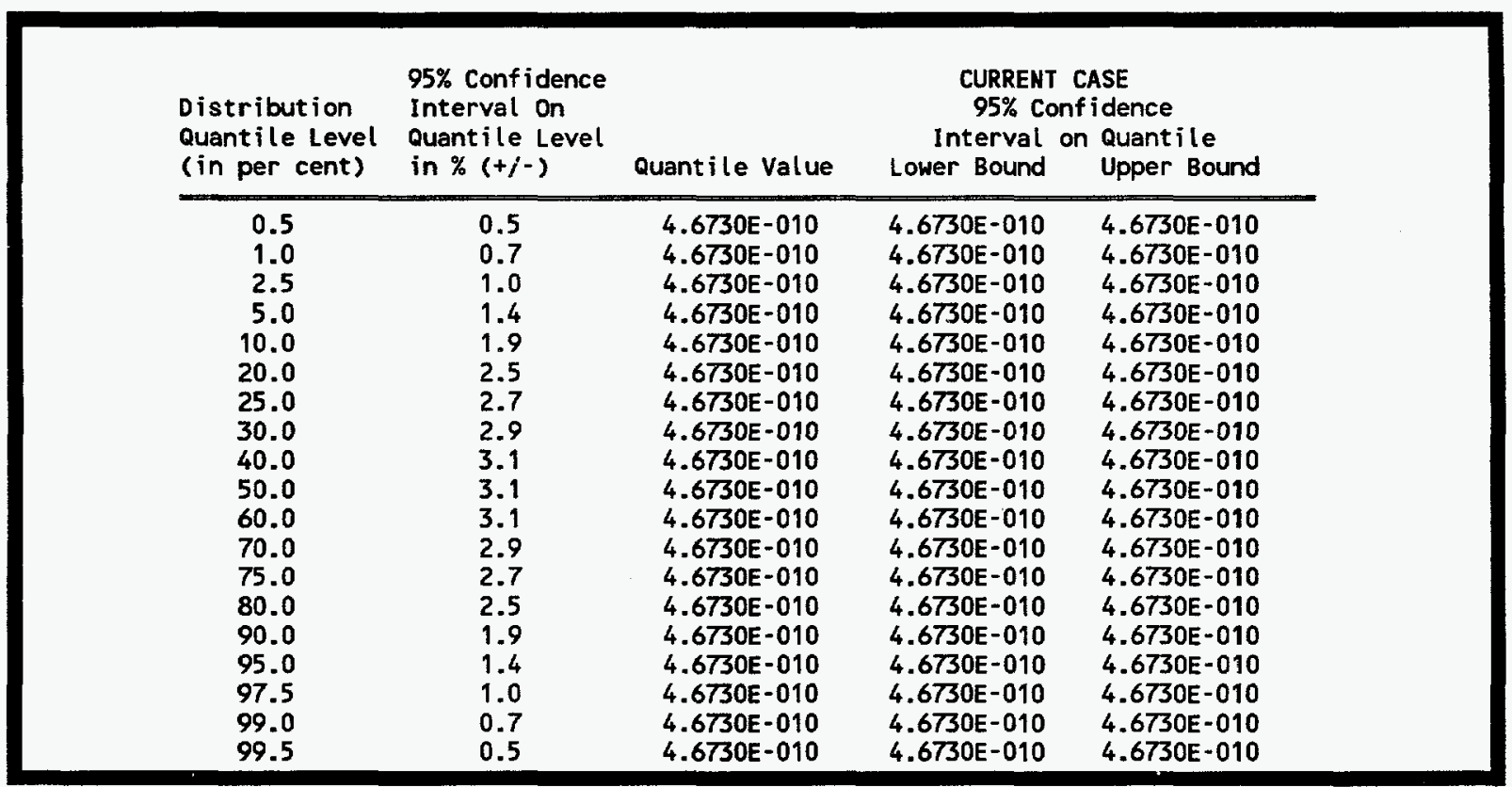

Figure 44. Current Quantile Values - example 
Condition Assessment

\subsection{Retrieving a Condition Assessment}

You can retrieve an existing condition assessment from the Name Event screen. This option lets you modify, copy, delete, and rename a resident condition assessment. See Section 3.2 for more details.

\subsection{Event Probability Changes Screen}

In the Event Probability Changes screen, you can change basic event current-case values without changing the base-case values. This screen allows you to change values for the Random Failure Data fields, Uncertainty fields, and the Process Flag field. See Section 3.3 for more details. 


\section{UTILITY}

The GEM Utility option allows one to perform routine functions that GEM requires such as defining constants, archiving the database, and recovering the database. Initiate this option as follows.

1. Type $<U>$ or highlight Utilities using the up and down arrow keys.

2. Press $<$ Enter $>$ to access the Utilities screen (Figure 45).

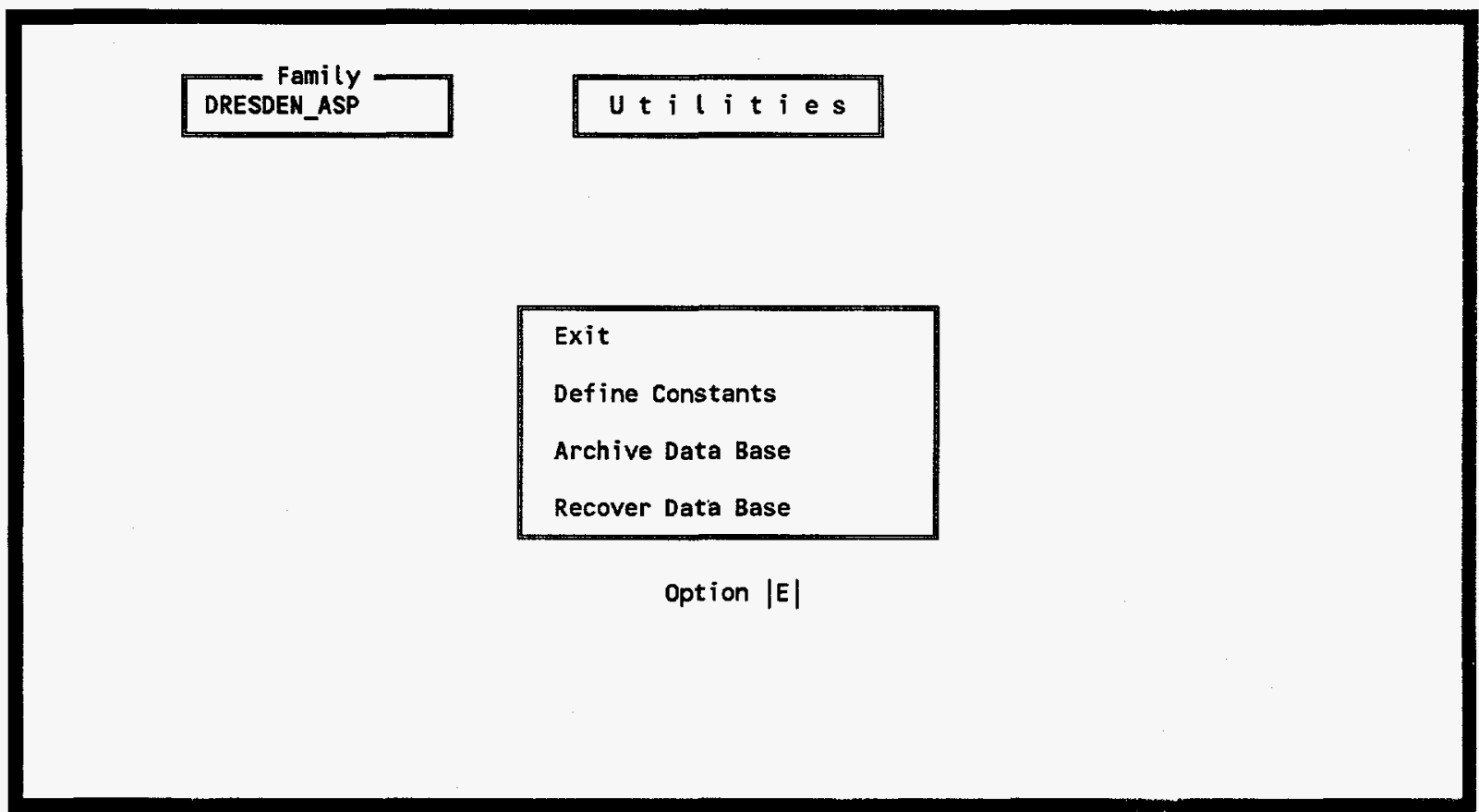

Figure 45. Utilities screen.

\subsection{Exit}

Exit returns you to the GEM main menu.

1. Type $\langle E\rangle$ in the Option field or highlight Exit.

2. Press $<$ Enter $>$ or press the $<$ Esc $>$ key to return to the GEM main menu.

NOTE: $\quad$ You can also press $<$ Esc $>$ to return to the GEM main menu.

\subsection{Define Constants}

The Define Constants option allows you to define what hardware the system uses as well as specifying file locations and archive information, and defining uncertainty settings, cut set constants, and default values for the graphics editor. 


\section{Utilities}

1. Type $<D>$ or highlight Define Constants using the up and down arrow keys.

2. Press <Enter $>$ to access the first Constants screen (Figure 46).

3. Enter the hardware information, file locations, and archive information. Table 1 provides a brief description of each field in the first Constants screen (Figure 46).

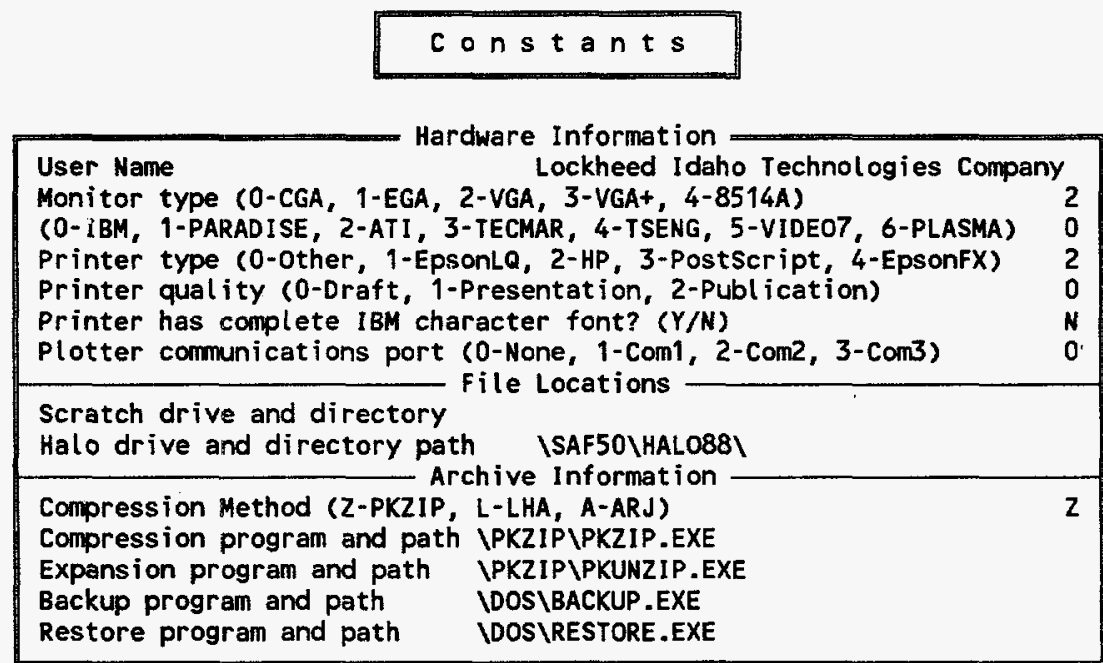

Change any of the constant values shown and press the <Enter> key.

Figure 46. Hardware information, file locations, and archive information provided in the first Constants screen. 
Utilities

Table 1. User Information field descriptions for constants option.

\begin{tabular}{|c|c|}
\hline FIELD & DESCRIPTION \\
\hline User Name & $\begin{array}{l}36 \text { character user identification (optional) } \\
\text { field }\end{array}$ \\
\hline Monitor type & $\begin{array}{l}0 \text { - Enhanced graphics monitor } \\
1 \text { - DEFAULT - Standard color graphics } \\
\quad \text { monitor } \\
2 \text { - Video graphics monitor }(640 \times 480) \\
3 \text { - Video graphics monitor plus }(800 \times 600) \\
4 \text { - } 8514 A\end{array}$ \\
\hline Card type & $\begin{array}{l}0 \text { - IBM } \\
1 \text { - Paradise } \\
2 \text { - ATI } \\
3 \text { - TECMAR } \\
4 \text { - TSENG } \\
5 \text { - VIDEO-7 } \\
6 \text { - PLASMA }\end{array}$ \\
\hline Printer type & $\begin{array}{l}0 \text { - Other } \\
1 \text { - Epson } \\
2 \text { - HP Laser (DEFAULT) }\end{array}$ \\
\hline Printer Quality & $\begin{array}{l}0 \text { - Draft } \\
1 \text { - Presentation } \\
2 \text { - Publication }\end{array}$ \\
\hline $\begin{array}{l}\text { Printer has complete IBM } \\
\text { character font? }\end{array}$ & $\begin{array}{l}Y=\text { Yes } \\
N=\text { No (DEFAULT) }\end{array}$ \\
\hline $\begin{array}{l}\text { Plotter communications } \\
\text { port }\end{array}$ & $\begin{array}{l}0 \text { - No Plotter connected to port (DEFAULT). } \\
1 \text { - Plotter connected to Com1 port. } \\
2 \text { - Plotter connected to Com2 port. } \\
3 \text { - Plotter connected to Com3 port. }\end{array}$ \\
\hline $\begin{array}{l}\text { Scratch drive and } \\
\text { directory }\end{array}$ & $\begin{array}{l}36 \text { character field indicating the drive } \\
\text { and path to the scratch directory where } \\
\text { files will be stored (DEFAULT set to blanks) }\end{array}$ \\
\hline $\begin{array}{l}\text { Halo drive and directory } \\
\text { path }\end{array}$ & $\begin{array}{l}36 \text { character field indicating the drive } \\
\text { and path to the Halo graphics that GEM } \\
5.0 \text { should use (IHALO88) }\end{array}$ \\
\hline Compression Method & $\begin{array}{l}\text { The compression method (package) you will } \\
\text { be using. } \\
\qquad \begin{array}{l}' Z \prime \\
' L '=\text { PKZIP } \\
' A \text { ' } A \text { ' }=\text { ARJ }\end{array}\end{array}$ \\
\hline $\begin{array}{l}\text { Compression program and } \\
\text { path }\end{array}$ & $\begin{array}{l}40 \text { character field indicating the drive, } \\
\text { path, and executable used to compress files. }\end{array}$ \\
\hline
\end{tabular}


Table 1. (continued)

\begin{tabular}{|l|l|}
\hline \multicolumn{1}{|c|}{ FIELD } & \multicolumn{1}{|c|}{ DESCRIPIION } \\
\hline $\begin{array}{l}\text { Expansion program and } \\
\text { path }\end{array}$ & $\begin{array}{l}40 \text { character field indicating the drive, } \\
\text { path, and executable used for file } \\
\text { expansion. }\end{array}$ \\
Backup program and path & $\begin{array}{l}40 \text { character field indicating the drive, } \\
\text { path, and executable used for backing } \\
\text { up files. }\end{array}$ \\
$\begin{array}{l}\text { Restore program and path } \\
\text { Size cutoff }\end{array}$ & $\begin{array}{l}40 \text { character field indicating the drive, } \\
\text { path, and executable used for restoring } \\
\text { files. }\end{array}$ \\
\hline
\end{tabular}

4. $\quad$ Press $<$ Enter $>$ to access the second Constants screen (Figure 47).

5. Enter the default values and flag settings for cut set generation, transformations, uncertainty and other miscellaneous information. Table 2 provides a brief description of each field in Figure 47.

$$
\text { Constants }
$$

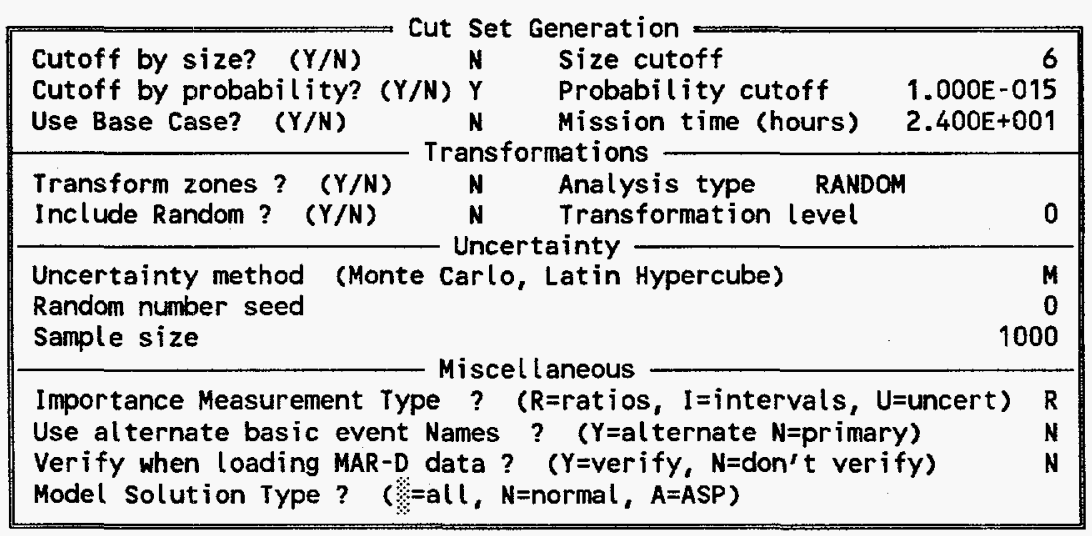

Change any of the constant values shown and press the <Enter> key.

Figure 47. Cut set generation, transformations, uncertainty and miscellaneous settings for the second Constants screen. 
Utilities

Table 2. Default values and flag settings.

\begin{tabular}{|c|c|}
\hline FIELD & DESCR IPTION \\
\hline Cutoff by size? & $\begin{array}{l}\text { Y - Do not generate fault tree or sequence } \\
\text { cut sets containing more basic events } \\
\text { than indicated in the size cutoff } \\
\text { field (DEFAULT). } \\
\text { N - Generate all cut sets for the fault } \\
\text { tree or sequence that meet the } \\
\text { probability cutoff criteria (if in } \\
\text { effect). }\end{array}$ \\
\hline Size cutoff & $\begin{array}{l}\text { The default maximum number of basic events } \\
\text { allowed in cut set generation when size } \\
\text { cutoff is in effect. DEFAULT }=6\end{array}$ \\
\hline Cutoff by probability? & $\begin{array}{l}Y \text { - Do not generate fault tree or sequence } \\
\text { cut sets that have a probability less } \\
\text { than the cutoff indicated in the } \\
\text { probability cutoff field (DEFAULT) }\end{array}$ \\
\hline & $\begin{array}{l}N \text { - Generate all cut sets that meet the } \\
\text { size cutoff criteria (if in effect) } \\
\text { regardless of the cut set probability. }\end{array}$ \\
\hline Probability cutoff & $\begin{array}{l}\text { The default minimum cut set probability } \\
\text { allowed in cut set generation when } \\
\text { probabil ity cutoff is in effect. } \\
\text { (DEFAULT }=1.00 E-015 \text { ) }\end{array}$ \\
\hline Mission time (hours) & $\begin{array}{l}\text { The default mission time to be used in the } \\
\text { calculation of basic event probabilities } \\
\text { (when appropriate). DEFAULT }=2.400 E+001\end{array}$ \\
\hline Importance Measurement & $\begin{array}{l}\text { R - Ratios (DEFAULT) } \\
\text { I - Intervals } \\
U \text { - Uncertainty }\end{array}$ \\
\hline $\begin{array}{l}\text { Use alternate basic } \\
\text { event names? }\end{array}$ & $\begin{array}{l}\text { Y - Alternate name will be used. } \\
\text { N - Primary name will be used (DEFAULT). }\end{array}$ \\
\hline Model Solution Type? & $\begin{array}{l}\text { Blank - Includes all models (DEFAULT) } \\
\text { N - Selects models defined as normal } \\
\text { A - Selects models defined as ASP }\end{array}$ \\
\hline
\end{tabular}

6. Press $<$ Enter $>$ three times to page through the third and fourth Constants screens and return to the Utilities screen. GEM does not use the information provided by these two screens (Figure 48 and Figure 49). 


\section{Utilities}

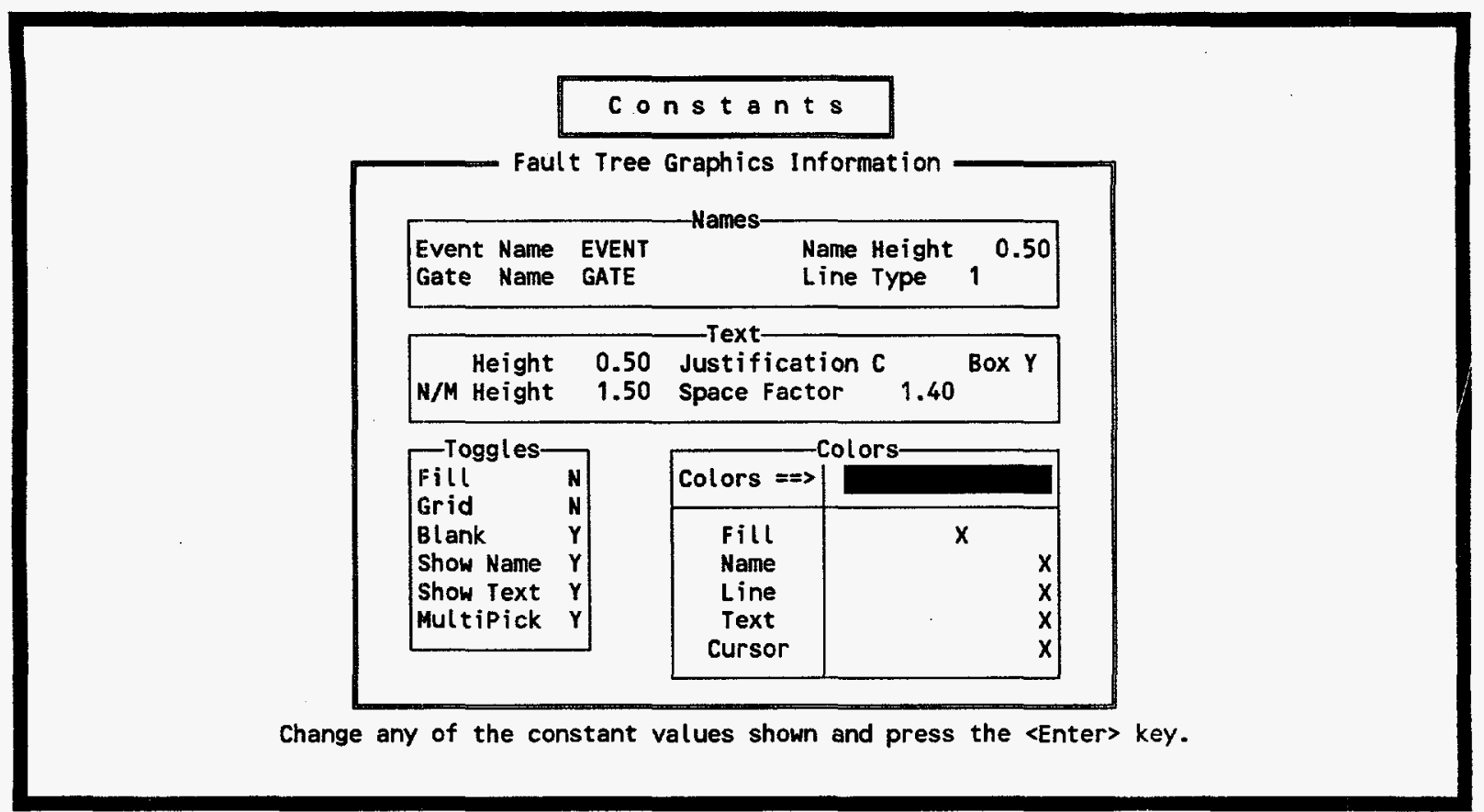

Figure 48. Fault tree graphics information for the third Constants screen.

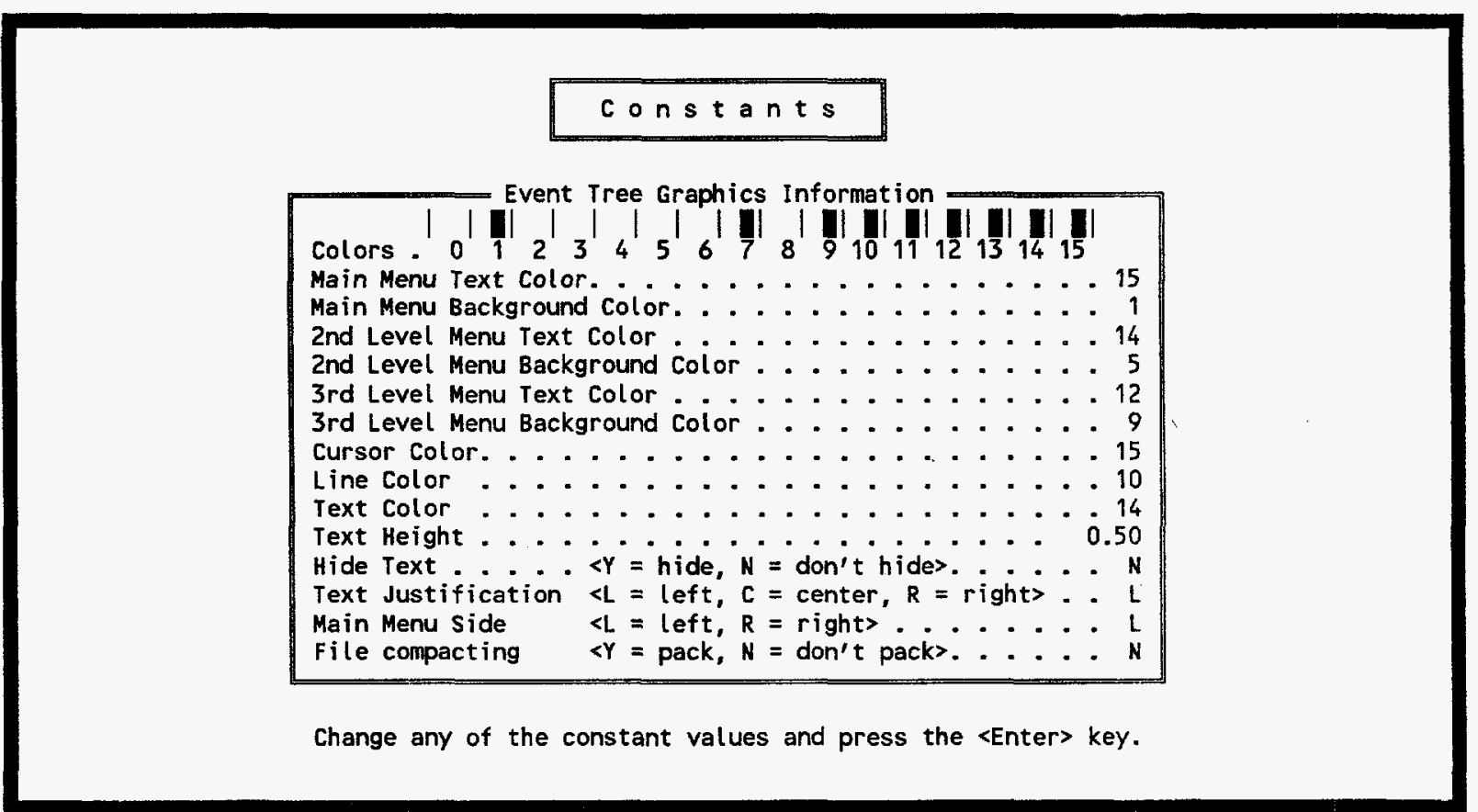

Figure 49. Event tree graphics information for the fourth Constants screen. 


\subsection{Archive Data Base}

The archive option allows you to compress, backup, restore, or expand data files for a family. Depending upon the status of the file, different options can be performed. Invoke this option as follows.

1. Type $<A>$ or highlight Archive Data Base using the up and down arrow keys.

2. Press $<$ Enter $>$ to access the Archive Family screen (Figure 50).

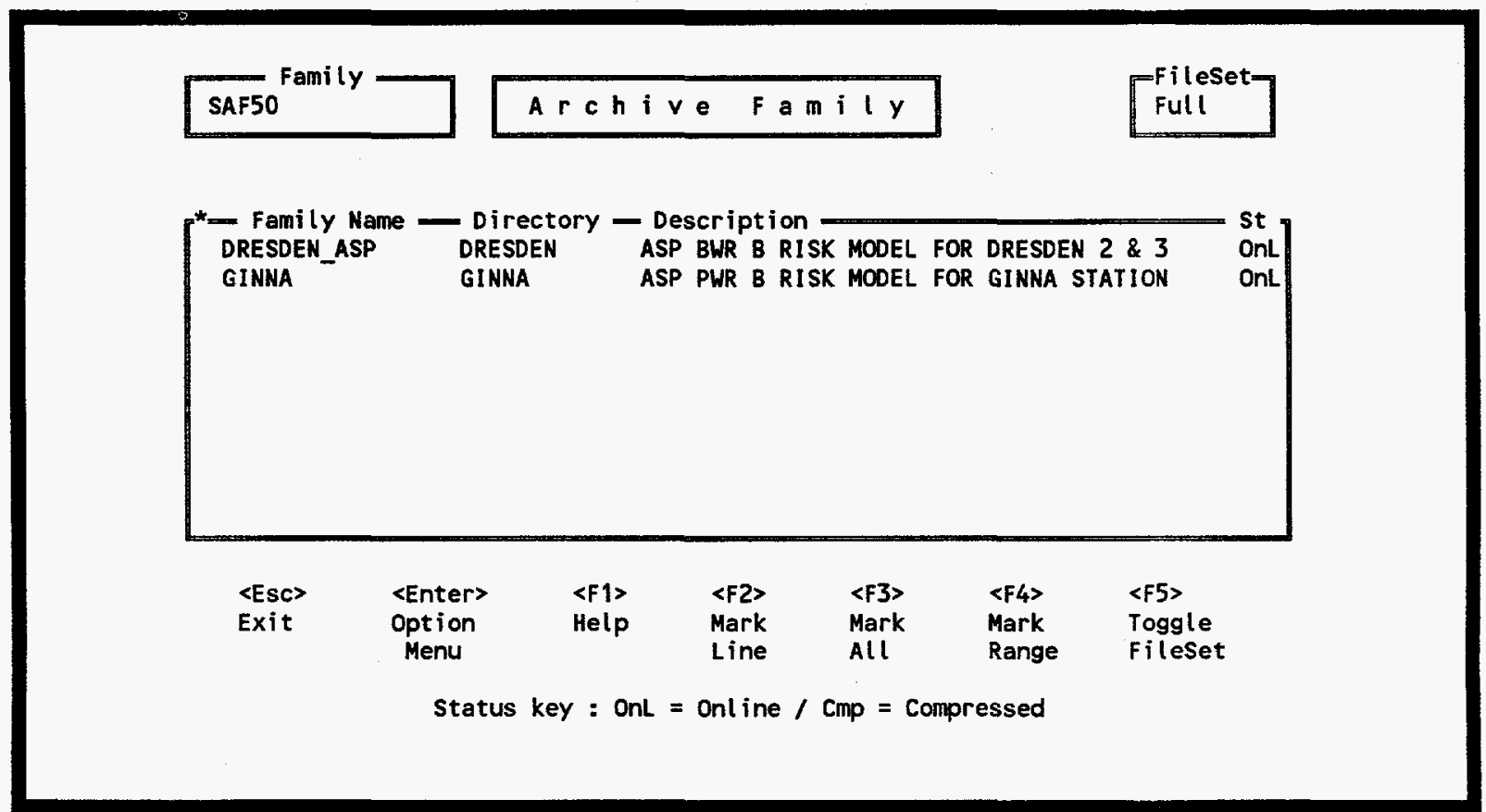

Figure 50. Archive Family screen.

This screen lists all the families currently defined, along with the directory name, description, and status. The status column can contain one of the following codes:

OnL The data files for the corresponding family exist online. The data files for these families can be accessed immediately.

Cmp GEM has compressed the data files for the family into an archive file. You must decompress these types of files before you can access them. GEM supports PKZIP, LHA (LHARC), and ARJ compression packages. The decompressed data files are deleted.

$\mathrm{CmB}$ GEM has compressed the data files for the family into an archive file and backed up the file on another medium. You must decompress and restore these types of files before you can access them.

Bck GEM has compressed the data files for the family backed up the file on another medium. 
Utilities

You must restore these types of files before you can access them.

Depending on the status of the data files, you can select from several different archiving options. These archiving options are discussed in the following paragraphs. The following function keys are available:

$<$ Esc $>\quad$ Exit-Terminates the archive option and returns you to the Utility menu.

<Enter > Option Menu-Displays the archiving options available. The archiving options are discussed in detail in Section 5.5.1.

$<$ F1 $>\quad$ Help-Displays online help messages.

$<$ F2 $>\quad$ Mark Line-Marks a family for further processing. See Section 1.8.2 for more details.

$<$ F3 $>\quad$ Mark All-Marks or unmarks ALL of the families for further processing. See Section 1.8.2 for more details.

$<$ F4 $\quad$ Mark Range-Marks a range of families for further processing. See Section 1.8.2 for more details.

$<$ F5 $>\quad$ Toggle Fileset-Toggles the file set (shown in the upper-right corner) from Full to Minimal. When you set the file set to full, GEM will use all files in the family directory in the subsequent archiving process. When you set the file set to minimal, GEM will use only the *.DFL, *.IDX, *.DAT, *.BLK, and *.CUT files in the subsequent archiving process.

\subsubsection{Archiving Options}

1. Select the desired families using the $\langle F 2\rangle,\langle F 3\rangle$, or $\langle F 4\rangle$ keys or highlighting the family using the up and down arrow keys.

2. Press $<$ Enter $>$ to access the Options window (Figure 51).

As shown, the following archiving options are available:

Compress. This option compresses the data files of the highlighted family and stores the compressed files in an archive file. GEM supports PKZIP, LHA, and ARJ. The decompressed data files are deleted. Invoke this option as follows.

1. Highlight Compress.

2. Press <Enter $>$. Upon completing the compression, the screen will display the message, "Compress Complete" and will return to the Archive Family screen. The status column (St) for the selected family(s) will change to $\mathrm{Cmp}$. 


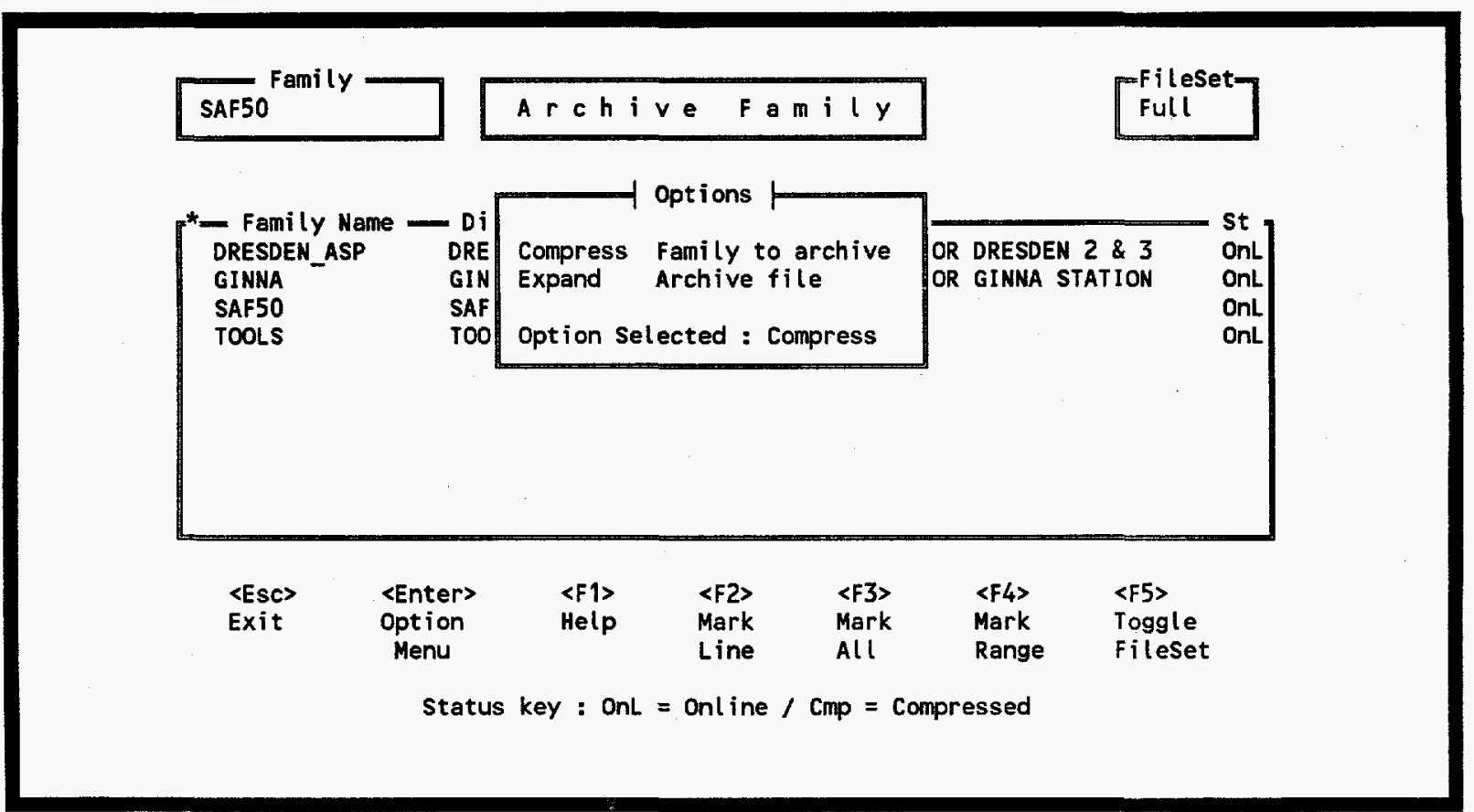

Figure 51. Options window.

Expand. This option extracts and expands the data files stored in the archive created by the Compress option described above. Initiate this option as follows.

1. Highlight Expand.

2. Press $<$ Enter $>$. Upon completing the expansion, the screen will display the message, "Expand Complete" and return to the Archive Family screen. The status column (St) for the selected family(s) will change to OnL.

NOTE: $\quad$ You must have marked a file with a status of Cmp for the expand option to work.

\subsection{Recover Data Base}

The Recover Data Base option performs all of the recovery options possible. This option first recovers the key indexes and then recovers all of the cross references. This option will take several minutes to complete. If your database has not been damaged, this option will restructure and optimize your database. Some indications that require a database rebuild include:

1. Data elements such as events/systems have been deleted and seem to reappear

2. During cut set generation or update, the min cut upper bound seems surprisingly high

3. Cross Reference reports show/don't show events being used properly

4. Events/systems that don't appear to be referenced cannot be deleted

5. After a software version update, and

6. GEM displays a message stating a data base recovery is required. 
Utilities

NOTE: Before recovering the database, backup the *.DFL files and corresponding data files (*.IDX, *.DAT, and *.BLK) in the directory of the family to be rebuilt.

You may recover the data base anytime because the recover process compacts the data and generally helps the software run faster.

1. Type $<R>$ or highlight Recover Data Base using the up and down arrow keys.

2. Press < Enter $>$ to access the Recover Data Base screen (Figure 52).

3. Type $\langle\mathrm{Y}\rangle$ to recover the data base or $\langle\mathrm{N}\rangle$ to return to the Utilities screen.

As shown, GEM performs several different recovery methods (Figure 53). Each method is discussed in the following paragraphs.

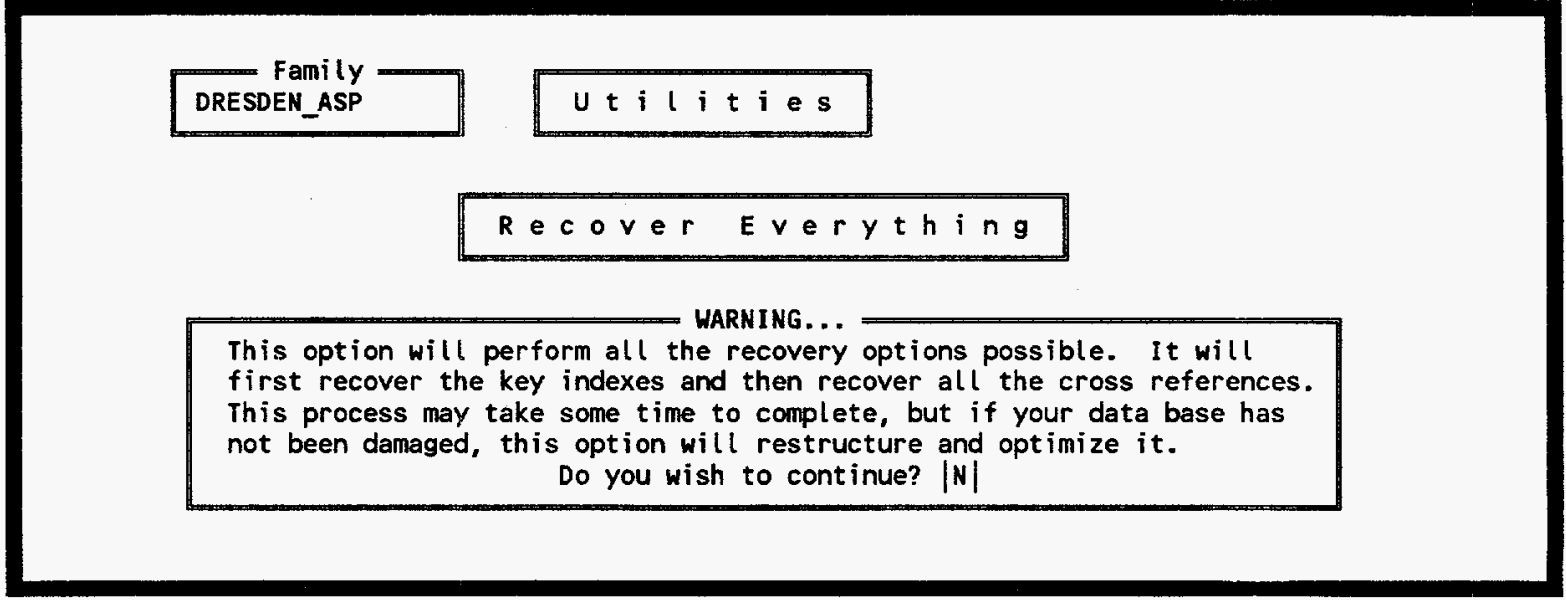

Figure 52. Recover Everything warning screen.

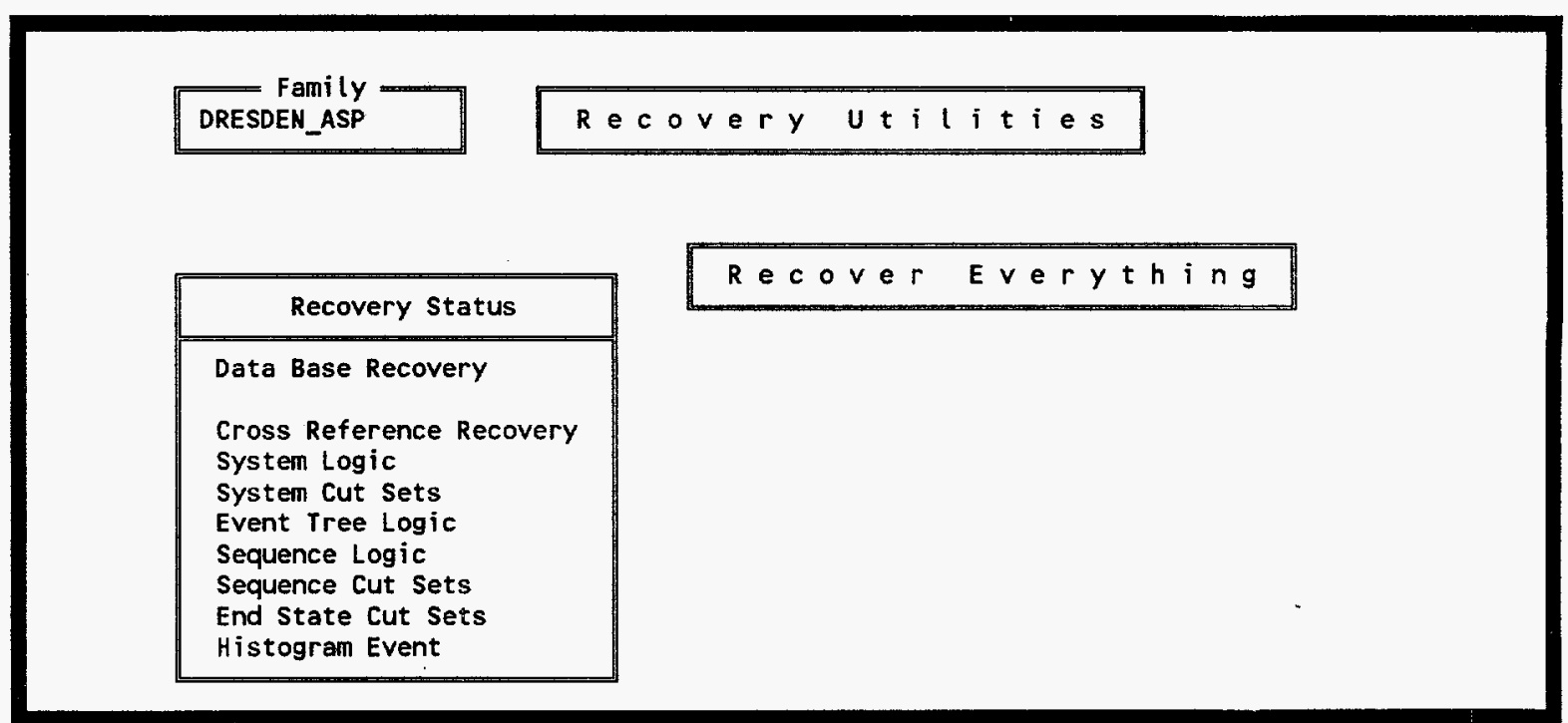

Figure 53. Recover Utilities screen. 


\subsubsection{Data Base Recovery}

The Data Base Recovery step rebuilds all of the files contained in your data base. If you think your data base has been damaged, using this step recovers all data files. If your data base has not been damaged, this option will restructure and optimize your data base.

\subsubsection{Cross Reference Recovery}

The Cross Reference Recovery step cross references all of the data contained in your data base.

\subsubsection{System Logic Events}

The System Logic Events step rebuilds the system logic events, gates, and transfers the crossreference list. GEM reads each logic record of the system and each event, gate, and transfer is noted. If no logic exists for a system, GEM will clear the list, and the screen will display a message and the recovery process will continue. If an event is referenced, but not used, it will be added.

\subsubsection{System Cut Set Events}

The System Cut Set Events step rebuilds the system cut set events cross reference list. GEM reads each base-case and current-case set of the system cut sets for each susceptibility type and notes each event used in those cut sets. If no cut sets exist for a system, The screen will display a message and the recovery process will continue. If an event is referenced but not used, it will be added.

\subsubsection{Event Tree Logic}

The Event Tree Logic step rebuilds the event tree logic cross reference list. This option reads the logic of each event tree graphic relation, noting any transfers in that logic. If a subtree is referenced, but does not exist, that subtree will be added to the data base.

\subsubsection{Sequence Logic}

The Sequence Logic step rebuilds the sequence logic cross reference list. GEM reads the logic of each sequence and notes each system used. If no logic exists, the screen will display a message and the recovery process will continue. This option also updates the EndState relation with the end states of each sequence.

\subsubsection{Sequence Cut Set Events}

The Sequence Cut Set Events step rebuilds the sequence cut set events cross reference list. GEM reads the base-case and current-case cut sets of each sequence for each susceptibility type and notes each event used in those cut sets. If no cut sets exist for a sequence, the screen will display a message and the recovery process will continue. If an event is referenced but not used, it will be added.

\subsubsection{End State Cut Sets}

The End State Cut Sets option rebuilds the end state cut set event cross reference list. GEM reads each base-case and current-case cut sets of each end state for each susceptibility type and notes each event used in those cut sets. If no cut sets exist for an end state, the screen displays a message on the screen and the recovery process will continue. If an event is referenced but not used, it will be added.

\subsubsection{Histogram Event}

The Histogram Event step rebuilds the histogram event cross reference lists. The program notes each histogram used by the basic events. 


\section{ERROR MESSAGES}

GEM displays messages at the bottom of the screen if it cannot complete a command or if GEM encountered an error with the database. The messages are listed in alphabetical order.

Each error message listed in this section is defined according to its meaning or cause. Each message also includes suggested corrections. When GEM detects an error while implementing a command, one of the following messages will appear at the bottom of the screen:

A sequence must be highlighted-You failed to highlight a sequence before processing the program command in the menu bar. Highlight the desired sequence by using the up and down arrow keys.

Access to family is denied-The family you have selected is password protected. Contact the system administrator for assistance.

Bad initiator-The system has encountered an error in the initiating event data base. Go back to the main menu and select Utilities. Highlight the Recover Data Base function to rebuild the data base. See Section $\mathbf{5 . 4}$ for more details about this function. If this fails, report the error to the system administrator.

Cannot add referenced endstate -- ABORT-This message occurs when GEM is recovering a family. Attempt to recover the family again. If this fails, report the error to the system administrator.

Change not allowed. Read-only attribute set-You attempted to change the probability of an event in an Event ID with read only access. You can only perform this change through IRRAS. If you do not have access to IRRAS change sets, contact your system administrator.

Class unknown - GEM did not recognize the class you attributed to the initiating event. Rebuild the data base. Select Utilities in the main menu and highlight the Recover Data Base function to rebuild the data base. See Section 5.4 for more details about this function. If this fails, report the error to the system administrator.

Compress failed-PKZIP encountered a problem while compressing the selected file. Several reasons exist for this type of failure. Possibly, one of the following errors occurred:

1. File does not exist or is not found.

2. You specified a local directory that does not exist on the system.

3. You mistyped the pathname or filename.

4. The destination hard drive or floppy diskette does not contain enough memory to store the designated file.

5. Insufficient memory. 


\section{Error Messages}

\section{Bad or illegal parameters were specified.}

Refer to your PKware manual for further information.

Cutset file access error-Failure occurred while reading cut sets. Select Utilities in the main menu and highlight the Recover Data Base function to rebuild the data base. See Section 5.4 for more details about this function. If this fails, report the error to the system administrator.

Data base was not closed correctly -- PLEASE RECOVER!!!-Several reasons exist for GEM encountering a damaged data base. One common reason for receiving this error occurs from improperly exiting out of GEM. Select Utilities in the main menu and highlight the Recover Data Base function to rebuild the data base. See Section 5.4 for more details about this function. If this fails, report the error to the system administrator.

Data base access error-Select Utilities in the main menu and highlight the Recover Data Base function to rebuild the data base. See Section 5.4 for more details about this function. If this fails, report the error to the system administrator.

Duplicate record exists, overwrite? (Y/N)-An assessment exists with that name. Press Y (yes) to overwrite the existing assessment with the new data. Press any other key to cancel the overwrite command. If you cancel this command, GEM will give you the option to enter a new assessment name.

Duplicate Event ID record exists-When you entered an Event ID in the Name Field, another Event ID with the same name already exists. Enter a new name in the field and press < Enter $>$.

Enter the event duration in hours-You left the duration field blank while exiting the Condition Assessment screen. Type the event duration into the field and press $<$ Enter $>$.

Enter an Event ID name-You tried to save the name event ID while leaving the Name field blank. Type an assessment name into the field and press < Enter $>$.

Error reformatting records...-An error occurred while trying to recover the data base. Report to system administrator.

Error Opening the Gate relation-GEM detects this error while trying to calculate an assessment. Data base may have been corrupted. Select Utilities in the main menu and highlight the Recover Data Base function to rebuild the data base. See Section 5.4 for more details about this function. If this fails, report the error to the system administrator.

Error Opening the Results relation-GEM detects this error while trying to calculate an assessment. Data base may have been corrupted. Select Utilities in the main menu and highlight the Recover Data Base function to rebuild the data base. See Section 5.4 for more details about this function. If this fails, report the error to the system administrator. 
Error Opening the System relation-GEM detects this error while trying to calculate an assessment. Data base may have been corrupted. Select Utilities in the main menu and highlight the Recover Data Base function to rebuild the data base. See Section 5.4 for more details about this function. If this fails, report the error to the system administrator.

Error saving new Event ID-Select Utilities in the main menu and highlight the Recover Data Base function to rebuild the data base. See Section 5.4 for more details about this function. If this fails, report the error to the system administrator.

Error displaying results-GEM cannot display the CCDP results. Report the error to system administrator.

Error creating report-Report this error to the system administrator.

Error initializing bit table-A lack of available memory could cause this error. Exit GEM and ensure that the minimum memory (RAM) is available to the application. Correct any problems associated with the memory. If this does not resolve the problem, report the error to the system administrator.

Error reading Eventree-While GEM was calculating an assessment, the data base may have been corrupted. Select Utilities in the main menu and highlight the Recover Data Base function to rebuild the data base. See Section 5.4 for more details about this function. If this fails, report the error to the system administrator.

Event ID name cannot be blank-When exiting the Rename Event ID screen, you erased the original name and left the Name field blank. To continue, enter a name for the event assessment.

Events must be marked or highlighted-You attempted to perform a function on an event before selecting an event. Move the cursor to the event, then select the desired option.

Expand failed-GEM encountered an error such as lack of disk space while compressing or expanding a family file. Report this error to the system administrator.

Family not found. Please check spelling-You tried to locate a family in the Select Family screen that does not exist in the Family Name list. Retype the family name. If you have typed the name correctly and it appears in the list, rebuild the data base. Select Utilities in the main menu and highlight the Recover Data Base function to rebuild the data base. See Section 5.4 for more details about this function. If this fails, report the error to the system administrator.

FATAL ERROR -- UNABLE TO OPEN-The GEM.DFL file does not reside in the proper directory or an error has corrupted the directory. Re-install GEM to correct this problem.

Highlight a family to select-You pressed the <Enter $>$ key before highlighting a family with the cursor. Select a family with the cursor and press $<$ Enter $>$. 


\section{Error Messages}

Invalid Analysis Type - Type not changed-In the Transformations section found in the second Constants screen (Figure 47), you entered an invalid analysis type into the Analysis Type field. Enter a valid analysis type into the field using one of the methods displayed in the Analysis Type window.

Invalid Option-You entered an invalid designator into the option field. Select one of the designators displayed in the option bar at the top of the form and press $<$ Enter $>$.

Invalid scratch path-In the File Locations section found in the first Constants screen, you entered an invalid scratch path in the Scratch Drive and Directory field. Check the path and enter a valid scratch path.

Logic not initialized-GEM possibly encountered a memory error. Exit GEM and ensure that the minimum memory (RAM) is available to the application. Correct any problems associated with the memory. If this does not resolve the problem, report the error to the system administrator.

Memory allocation error-The computer does not have enough free RAM memory to execute the program or command. Exit GEM and ensure that the minimum memory (RAM) is available to the application. Correct any problems associated with the memory. For example, remove any unneeded device drivers or RAM-disk software from the CONFIG.SYS file and restart DOS. If this does not resolve the problem, report the error to the system administrator.

No saved Assessments exist-You pressed F2 to retrieve an assessment; however, no saved assessments exist for the selected model.

No sequences effected-This message occurs while GEM is calculating an assessment. This may or may not indicate that the data base has been corrupted. If you suspect that the data has been corrupted, rebuild the data base. Select Utilities in the main menu and highlight the Recover Data Base function to rebuild the data base. See Section 5.4 for more details about this function. If this fails, report the error to the system administrator.

No nonzero sequences-The screen will only display nonzero sequences for the CCDP. This message occurs when the changes to the basic events produce results only equaling zeroes. If you think this is an error, rebuild the data base. Select Utilities in the main menu and highlight the Recover Data Base function to rebuild the data base. See Section 5.4 for more details about this function. If this fails, report the error to the system administrator.

No probability changes are defined-If events with changes appear on the screen and you receive this message when attempting to reset the event to its base-case value, report the error to the system administrator. 
No family record found-This message occurs while attempting to display the list of available families. Exit GEM and install the ASP models. Then, check the directory structure. The base directory should exist as ISAF50 $\backslash$ and contain the subdirectory $\backslash$ TOOLS $\backslash$ as well as a subdirectory for each family. Correct any discrepancies.

No initiating events found-GEM could not locate any initiating events in the fault tree for the selected family while attempting to perform an Initiating Event Assessment. This may or may not be an error. If you think this is an error, recover the family. Select Utilities in the main menu and highlight the Recover Data Base function to rebuild the data base. See Section 5.4 for more details about this function. If this fails, report the error to the system administrator.

No Plant Data-The model still requires a conversion to work with the latest version of GEM. Rebuild the data base. Select Utilities in the main menu and highlight the Recover Data Base function to rebuild the data base. See Section 5.4 for more details about this function. If this fails, report the error to the system administrator.

No version control schema found-A dfl file is missing in the $\mid$ tools $\backslash$ directory. Re-install GEM.

Option unavailable-You pressed an invalid option from a menu screen. Choose an option from the menu.

Out of memory error-The computer does not have enough free memory (RAM) to execute the program or command. Exit GEM and ensure that the minimum memory (RAM) is available to the application. Correct any problems associated with the memory. For example, remove any unneeded device drivers or RAM-disk software from the CONFIG.SYS file and restart DOS. If this does not resolve the problem, report the error to the system administrator.

Selected option not available-You entered an invalid designator into the option field. Select one of the designators displayed in the option bar at the top of the form and press $<$ Enter $>$.

Sequence table initialization error-The screen will present this message while displaying the CCDP results. The computer might contain insufficient memory to complete this function. Exit GEM and ensure that the minimum memory (RAM) is available to the application. Correct any problems associated with the memory. For example, remove any unneeded device drivers or RAM-disk software from the CONFIG.SYS file and restart DOS. If this does not resolve the problem, report the error to the system administrator.

Sort table initialization error-The screen will present this message while displaying the CCDP results. The computer might contain insufficient memory to complete this function. Exit GEM and ensure that the minimum memory (RAM) is available to the application. Correct any problems associated with the memory. For example, remove any unneeded device drivers or RAM-disk software from the CONFIG.SYS file and restart DOS. If this does not resolve the problem, report the error to the system administrator.

Unable to select desired family-This error occurs while trying to select a family in the Select Family screen. Report this error to the system administrator. 
Error Messages

Unable to change directories to entrance location-This error occurs after expanding a family. Report this error to the system administrator.

Unable to change directories to data location-Report this error to the system administrator.

Unable to execute program in compress-The system was unable to locate either the ZIPTOEXE.EXE file or the PKZIP.EXE file. Go to the Define Constants option (Section 5.2) in Utilities and verify in screen 1 that the Archive Information section is correct. If this information is correct and you use PKZIP as your archive program, then ensure that ZIPEXE.EXE resides in the same directory as the PKZIP file.

Unable to execute program in expand-The system was unable to locate the PKUNZIP.EXE file. Go to the Define Constants option (Section 5.2) in Utilities and verify in screen 1 that the Archive Information section is correct.

Unable to rebuild the family-The system was unable to rebuild the family while the system was trying to recover the data base. Report this error to the system administrator.

Unable to open the requested report file-An error occurred while creating a report. Ensure that you have entered a valid file name (drive and directory) in the Name field. Also ensure that the disk drive has enough available disk space.

Unexpected error-If you encounter this error, recover the data base. Select Utilities in the main menu and highlight the Recover Data Base function to rebuild the data base. See Section 5.4 for more details about this function. If this fails, report the error to the system administrator.

Version mismatch...-While GEM is recovering a family, GEM encountered a mismatch between data base versions. Attempt to recover the family again. If this fails, data models may be based on a newer schema. Check version dates.

Warning: Expected event not found, prob not changed:-If you encounter this error, recover the data base. Select Utilities in the main menu and highlight the Recover Data Base function to rebuild the data base. See Section 5.4 for more details about this function. If this fails, report the error to the system administrator.

WordPerfect format requires a valid file name-While in the Report option, you selected " $\mathrm{Y}$ " for the WordPerfect format; however, you did not enter a file name. Type a valid file name (drive and directory) in the field and press < Enter $>$. "CON" and "PRN" are not valid file names. 


\section{Appendix A}

Hardware Requirements and Installation Procedures 



\section{Appendix A}

\section{Hardware Requirements and Installation Procedure}

As a minimum, GEM 5.0 requires the following hardware configuration:

IBM compatible 386 with $640 \mathrm{~K}$ main memory, $2 \mathrm{MB}$ extended memory

DOS 3.3 or later

20M hard disk (minimum)

Math co-processor (optional)

16 color enhanced monitor (EGA or better)

graphics input devices - keyboard or mouse.

If an enhanced graphics adapter is used, it must have the memory expansion option to extend the standard 4 colors to 16. This option is an upgrade to the IBM EGA board, but is usually standard on boards manufactured by other vendors. GEM does not support the 4-color mode on the EGA adapter.

The recommended configuration contains a VGA color monitor and adapter, a mouse as the graphics input device, and a math co-processor. The keyboard can serve as the graphics input device but is not nearly as user-friendly as the mouse. GEM will not run as fast if a math co-processor is not present.

NOTE: $\quad$ GEM 5.0 requires the above specified amount of random access memory (RAM). This memory must be available for the program and not used by memory resident programs. Also, certain memory resident programs may interfere with the execution of GEM. These should be unloaded before using GEM. The MS/DOS command "MEM" can be used to display the amount of memory available on your machine.

To install GEM 5.0 on your computer, perform the following steps:

1. Insert the GEM diskette, and type

$$
\text { x:install and press }<\text { Enter }>
$$

where $\mathrm{x}$ is the drive containing the installation diskette(s).

Figure A-1 appears. On this screen, fill out the following information:

Source drive $\quad-\quad$ Enter the drive containing the installation diskettes.

Destination Drive $\quad-\quad$ Enter the drive where the GEM files will be installed.

Destination Directory - $\quad$ Enter the directory name where the GEM program files will be written. The specified destination directory will be created in the root directory of the destination drive. 


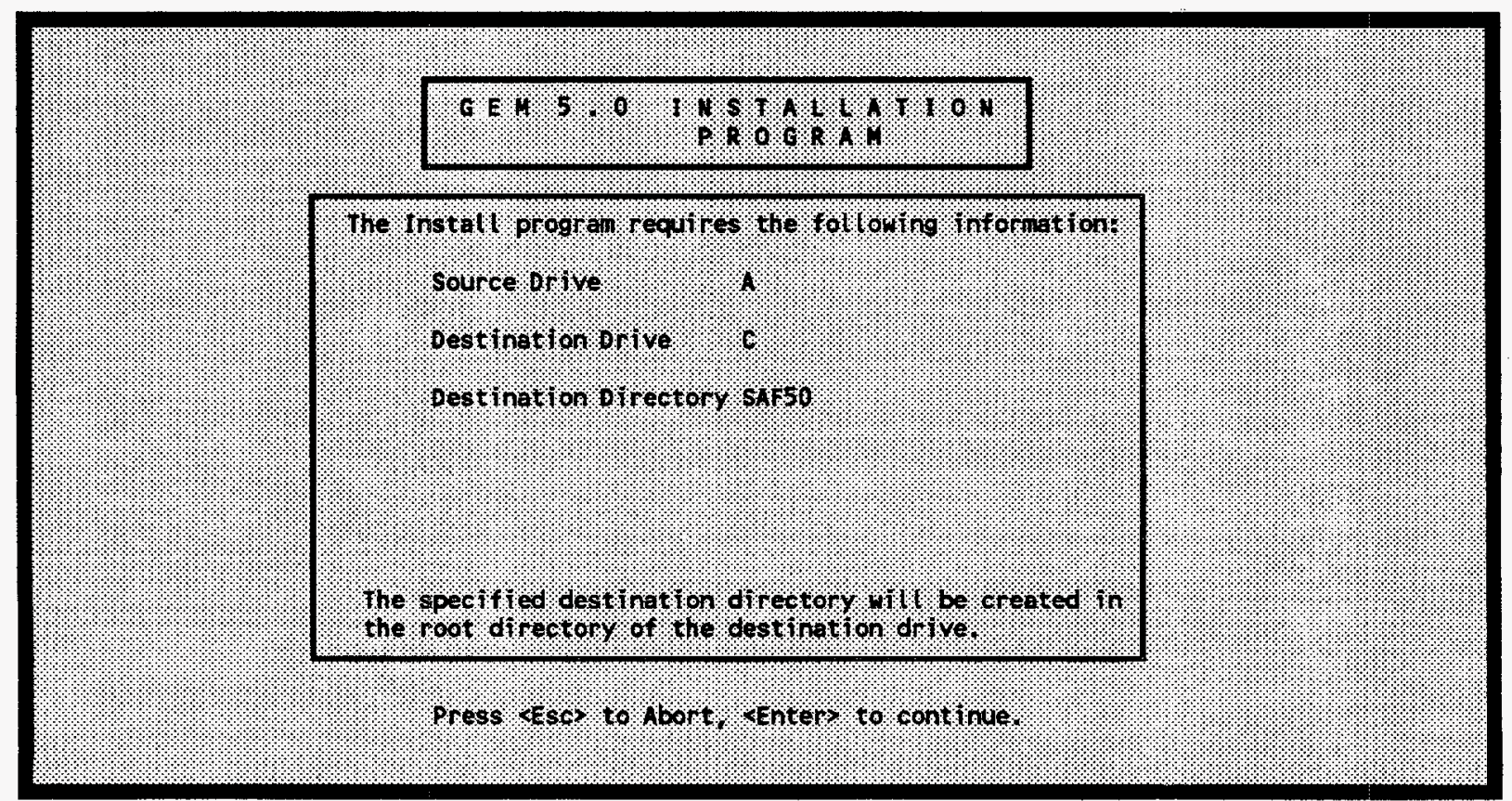

Figure A-1. GEM 5.0 Installation Program screen.

When complete, press < Enter $>$ to continue.

2. The program files on the installation diskette is compressed. The installation procedure de-compresses these files and copies them onto the hard drive. The de-compression process is indicated by the listing of files being "exploded" or "inflated". These terms are used by the de-compression software to indicate the process used when the files were compressed.

3. When complete, you will be returned to the destination directory.

NOTE: If you are loading GEM from a network, you will not be loading diskettes; however, the process is the same.

After the installation is completed, you need to locate and modify your CONFIG.SYS file. This file is in the root directory. If you do not have a file by this name, you must create one. In either case, the following parameters must be included in the file, if not already present.

$$
\begin{aligned}
& \text { FILES }=40 \\
& \text { BUFFERS }=15 \\
& \text { DEVICE }=\text { ANSI.SYS }
\end{aligned}
$$

The device driver ANSI.SYS must also be copied from the DOS directory to the root directory if it is not already there. 
The installation procedure will create a batch procedure, GEM.BAT, for executing GEM 5.0 in the ISAF50 directory. This procedure may be used directly or adapted to meet your specific needs. If you choose not to modify the procedure, the following format is used to execute GEM 5.0. Type:

\section{CDISAF50}

\section{GEM}

This completes the installation of the GEM 5.0 software. You must now ensure that the proper graphics input device is hooked up and ready for use. When this is done, the GEM 5.0 system is ready for use. Refer to Section 5 for a discussion on defining constants for your configuration. 
Appendix B

Gem Technical Reference 



\section{Appendix B \\ GEM Technical Reference}

\section{B-1. INTRODUCTION}

The GEM software module has been designed as a highly specialized user interface to the SAPHIRE suite of PRA codes for the evaluation of operational events at commercial nuclear power plants. In particular, GEM implements many aspects of the Accident Sequence Precursor (ASP) program analysis methods and can only use GEM with the ASP program models or other models built to the same specifications. Initiating event assessments and condition assessments are both addressed, each with its own set of unique requirements. The calculation of conditional core damage probabilities (CCDPs) and event importances are automated as much as possible.

This appendix provides some technical background information so that the user can obtain a basic understanding of what GEM does, how GEM works, and why GEM performs the tasks the way GEM does. First, some event evaluation concepts and definitions are presented, then initiating event assessments are discussed, and finally, condition assessments are addressed.

\section{B-2. DEFINITIONS AND CONCEPTS}

\section{B-2.1 Definitions}

Appendix B uses the following terms throughout the report:

- $\quad$ Accident Sequence Precursor (ASP). In general, an accident sequence precursor is a sequence of events that successfully prevented core damage, that if additional failures had occurred, would have resulted in core damage. Precursors have been separated into two types. The first type involves the occurrence of an initiating event (analyzed in an initiating event assessment), and the second type involves failures or degradations in mitigating equipment (analyzed in a condition assessment). In the ASP program, a precursor is retained if the likelihood of those additional failures leading to core damage is greater than or equal to 1.0E-6. Occasionally, exceptions exists for events with coincident containment failure or unique influences difficult to analyze. These are reported in the ASP program annual reports but do not necessarily receive the same rigorous analytical treatment as the more traditional precursors.

- $\quad$ ASP Model. An ASP model is a SAPHIRE-based probabilistic risk assessment (PRA) event treefault tree model designed specifically for performing event assessments. These models are slightly different than the typical PRA models in several areas. The initiating event frequencies are expressed as per hour values to more readily facilitate event durations normally expressed in hours. Some initiating events have associated short-term recovery factors not found in typical PRA models. Because the models may be used to evaluate the impact of failure of highly reliable components, low probability minimal cutsets can become dominant. Therefore, the recovery actions must be applied evenly across the entire set of minimal cutsets and not just the dominant ones. 
- Change Set. A change set is a listing of the risk model basic events that have been designated for a change in probability from the base case. In ASP evaluations, a change set is used to identify the basic events (and initiating events) that must be re-evaluated to represent the events and conditions observed during the reported operational event. GEM assists in creating the proper ASP evaluation change set by automatically identifying basic events that must change once the initiating event or condition duration is identified.

- Conditional Core Damage Frequency (CCDE). The conditional core damage frequency is the hazard rate representing the expected number of core damage events per hour given a set of known failures or plant operating conditions.

- Conditional Core Damage Probability (CCDP). The conditional core damage probability is the likelihood of experiencing a core damage event given a set of known failures or plant operating conditions. When calculated for an operational event, the CCDP is a measure of how close the plant came to core damage during the event. Alternatively, the CCDP can be thought of as the likelihood of failure of the remaining barriers to core damage. The CCDP is calculated by GEM using the following equation:

$$
C C D P=1-e^{-C C D F * t}
$$

- $\quad$ Core Damage Frequency (CDF). The core damage frequency associated with the base case results of an ASP model is comparable to that of a typical PRA except it is expressed on a per hour basis. It is a hazard rate representing the expected number of core damage events per hour.

- $\quad$ Core Damage Probability (CDP). For a condition assessment, the core damage probability is the likelihood of experiencing a core damage event within an exposure time, $t$, given that all plant and system responses are allowed to fail at their nominal failure rates. In the past, the ASP program estimated the CDP as

$$
C D P=C D F * t
$$

This is only an approximation to the actual exponential equation now being used by GEM:

$$
C D P=1-e^{-C D F * t}
$$

- Event Importance. The event importance is a measure of how much greater the core damage probability for the analyzed event was compared to the nominal core damage probability. It is similar to a risk increase difference importance measure. The Event Importance is calculated in GEM by 


$$
I=C C D P-C D P
$$

GEMDATA. GEMDATA is a software package that assists the ASP model developers with the calculation of the various electric power non-recovery probabilities and the reactor coolant pump (RCP) seal loss of coolant accident (LOCA) probability contained in the ASP models.

\section{B-2.2 Concepts}

\section{B-2.2.1 ASP Models}

ASP models are specifically designed to perform ASP evaluations of operational events. The features unique from the typical PRA model include:

- Initiating event frequencies are expressed on a per hour basis to facilitate condition assessments

- $\quad$ For the loss of offsite power (LOOP) model, the specific type of LOOP event experienced must be analyzed. The ASP models can address any of four different LOOP events: (1) grid-related, (2) plant-centered, (3) severe weather, and (4) extremely severe weather. Each type has its own initiating event frequency, probability of non-recovery curve, and probability of reactor coolant pump (RCP) seal failure curve. The base case model uses frequency-weighted average values for the LOOP initiating event frequency, non-recovery values, and RCP seal failure probability.

\section{B-2.2.2 Initiating Event Assessments}

ASP models contain event trees that model the plant response to a selected set of initiating events. When an operational event to be analyzed involves one of these initiating events, an initiating event assessment is performed. The analyst enters GEM and after selecting the correct plant model, selects an initiating event assessment and gives it a name. This opens a work space for this particular initiating event assessment.

The analyst next selects the initiating event that occurred from the list provided. GEM will automatically set the selected initiating event's frequency to its pre-assigned, short-term, non-recovery probability (or 1.0 if recovery is deemed not possible) and will set all other initiating event frequencies to zero. This assumes that the actual operational event involves only the observed initiating event and all other initiators are not applicable. Furthermore, it is assumed that the probability of another initiating event occurring while the first initiating event is still in progress is small.

If the initiating event is a LOOP, GEM will display a list of the four different types of LOOP (plant-centered, grid-related, severe weather-related, and extremely severe weather-related) and the analyst must pick one. Each LOOP type has a different short-term, non-recovery probability. 


\section{GEM Technical Reference}

Once the initiating event is selected, GEM displays a predefined change set unique to that initiating event. For all initiating events except LOOP, the change set consists only of the entire set of initiating events. The selected initiating event is in the change set because its frequency has been converted to a probability equal to its short-term, non-recovery value and the other initiating events are listed because their frequencies have been converted to a probability of zero.

For the LOOP initiating event, the change set again lists all the initiating events, but additionally, the change set includes a predefined list of electric power recovery basic events and the RCP seal LOCA basic event (for PWRs only). These are the basic events that must be changed when going from the nominal LOOP event in the base case model to a specific LOOP type for the event assessment. These basic events are automatically added to the change set and their values are automatically modified to predetermined values based on the plant characteristics, the timing required, and the type of LOOP. See the discussion on GEMDATA for more details.

The analyst can access any of the basic events listed in the change set if a need exists to make modifications. Other basic events can also be added to the change set list. This would be necessary to model any observed failures or degradations reported for the event being analyzed.

Once the change set list is complete and the desired changes are defined for the basic events, the analyst selects the PROCESS option. GEM automatically generates a current basic event data set according to the changes defined in the change set. GEM next determines which system fault trees have been impacted by the changes and identifies the event tree accident sequences that use those fault trees. The logics for those sequences are loaded into memory and GEM solves the logic for the new minimal cutsets. The minimal cutsets are quantified, the CCDP is calculated and the results are displayed. With the exception of the CCDP calculation, GEM processes the accident sequences in exactly the same manner as IRRAS.

\section{B-2.2.3 Condition Assessments}

When an operational event does not involve the actual occurrence of an initiating event, but does indicate failures or degradation of equipment that would be required to mitigate the consequences of an initiating event, a condition assessment is performed. After entering GEM and selecting the appropriate ASP model, the analyst selects the condition assessment option and gives the assessment a name. This opens up a work space for this particular condition assessment. GEM then prompts the analyst for the duration of the conditions being analyzed. The duration is entered, expressed in hours.

GEM displays a blank change set and provides the ability to add events to the change set to model the observed failures and degradations reported for the event being analyzed.

Once the change set list is complete and the desired changes are defined for the basic events, the analyst selects the PROCESS option. GEM automatically generates a current basic event data set according to the changes defined in the change set. GEM next determines which system fault trees have been impacted by the changes and identifies the event tree accident sequences that use those fault trees. The logics for those sequences are loaded into memory, GEM solves the logic for the new minimal cut sets, and quantifies them.

For the sequences that were not impacted by any of the changes, the base case results are copied over into the current case results. If you attempt to look at the current case cut sets for one of these sequences you will get the message, "No cut sets to process." The CCDP is calculated and the results are displayed. With the 


\section{GEM Technical Reference}

exception of the CCDP calculation, GEM processes the accident sequences in exactly the same manner as IRRAS.

\section{B-2.3 GEMDATA Interactions}

\section{B-2.3.1. Information Provided by GEMDATA}

To more adequately and easily model the actual conditions associated with a LOOP, a database management and computation system, GEMDATA, was developed. GEMDATA is not a part of GEM and is not included in the standard distribution of the SAPHIRE software package. GEMDATA is a successor to an earlier code developed by SAIC for the ASP program called BLACKOUT ${ }^{1}$. GEMDATA was developed to provide the BLACKOUT code capabilities in a format compatible with GEM and to provide more flexibility in managing the database information.

GEMDATA stores and processes the information necessary to determine the proper short-term and long-term offsite power non-recovery values and the probability of an RCP seal LOCA given a LOOP. Each of these parameters are calculated for the four types of LOOP: grid-related, plant-centered, severe weather, and extremely severe weather. Additionally, the database stores the short-term, non-recovery values for the other initiating events as well.

The following information contained in the database is arranged by plant:

- Plant-centered LOOP class. Each plant has been assigned to one of three plant-centered LOOP classes based on the expected frequency of experiencing a plant-centered LOOP. Class 11 has the lowest frequency for a plant-centered LOOP of a given duration. Class I3 has the highest frequency and Class $\mathrm{I} 2$ is roughly half way in between the other two. These classes are defined and further explained in NUREG-1032 2 .

- Grid class. Each plant has been assigned to one of four grid classes based on the reliability of the offsite power grid. Class Gl has the lowest frequency of grid loss (less than 1 per 60 site-years) and Class G4 has the highest frequency of grid loss (greater than 1 per 6 site-years). Classes G2 and G3 have grid loss frequencies between the other two. See NUREG-1032 for more details.

- Grid recovery type. Each plant is assigned to one of two grid recovery types. Type Rl is assigned to plants that have the capability and procedures to recover offsite (nonemergency) AC power to the site within 0.5 hour following a grid blackout. Type $\mathrm{R} 2$ is assigned to all other plants. See NUREG1032 for more details.

- Severe weather class. Each plant is assigned to one of five severe weather classes based on the frequency of experiencing a loss of offsite power due to severe weather conditions. Severe weather conditions include lightning, rain, hail, sleet, snow, moderately high winds, and other weather-related causes that do not greatly affect the time to restore power. Class S1 has the lowest frequency of offsite power loss (less than 1 per 333 site-years) and Class S5 has the highest frequency (greater than 1 per 10 site-years). See NUREG-1032 for more details. 
- Severe weather recovery type. Each plant is assigned to one of two severe weather recovery types. Type $\mathrm{R} 1$ is assigned to plants that have the capability and procedures to recover offsite (nonemergency) AC power to the site within 2 hours following a severe-weather-induced loss of offsite power. Type $R 2$ is assigned to all other plants. See NUREG-1032 for more details.

- Extremely severe weather class. Each plant is assigned to one of five extremely severe weather classes based on the frequency of experiencing a loss of offsite power due to extremely severe weather conditions. Extremely severe weather conditions include tornadoes, hurricanes, very high winds, large accumulations of snow and ice, and other weather-related causes that create conditions so that power cannot be restored for a long period of time. Class SS1 has the lowest frequency of loss of offsite power (less than 1 per 3333 site-years) and SS5 has the highest frequency (greater than 1 per 100 site-years). See NUREG-1032 for more details.

- RCP seal design. Each PWR plant is assigned to one of seven RCP seal design categories based on the plant configuration for resisting and mitigating a seal LOCA given a loss of offsite power. BWR plants do not have entries in this field in the database. The seal LOCA probability models are based on the NUREG- $1150^{2}$ work and include models for the following designs: Westinghouse old O-ring, Westinghouse new $\mathbf{O}$-ring, and seal return isolation. Additionally, models have been developed to simulate a select set of unique modeling conditions. These include: seals never fail, seals fail in 0.5 hours, seals fail in 1 hour, and seals fail in 2 hours.

- Core uncovery time. This is the time it takes to uncover the top of active fuel from a complete loss of core cooling and injection based on the maximum decay heat rate. This value is nominally set to 0.5 hours.

- Battery depletion time. This is the time it takes to suffer a complete loss of DC power following a station blackout. This time is based on the battery capacity, the expected DC power requirements, and the plant procedures for load shedding. This information has been gathered from the station blackout rule responses and other plant information such as FSARs, IPEs, and PRAs.

- Diesel repair time. This is the median time for restoration of one diesel generator when more than one are unavailable due to independent faults. A median time of 4 hours is nominally used based on information in NUREG-1032.

- Short-term nonrecovery time, $\mathbf{t}_{\text {short }}$. For initiating events that are recoverable (currently only small LOCAs and LOOPs are considered recoverable), recovery within this time would terminate the specific initiating event response. The general transient response would then be appropriate. A rigorous treatment of these recovered initiating events would transfer to the transient event tree; however, the sequence frequencies would be much smaller than the existing transient sequence frequencies and are ignored. The short-term nonrecovery time is generally 0.5 hours.

- Non-LOOP initiating event nonrecovery values. Transients and steam generator tube ruptures are considered nonrecoverable and are assigned a nonrecovery value of 1.0. Small LOCAs are recoverable within time $t_{\text {short }}$. The probability of not recovering within $t_{\text {short }}$ for BWRs is 0.5 and 0.43 for PWRs based on information provided in NUREG/CR- $4674^{3}$. 
GEMDATA uses this information, along with associated equation parameters, to calculate the various LOOP initiating event frequencies, short-term non-recovery values, long-term recovery values, and RCP seal failure probabilities.

\section{B-2.3.2 LOOP and Seal LOCA Probabilities}

B-2.3.2.1 LOOP Frequencies. NUREG-1032 developed curves of LOOP frequency as a function of LOOP duration for each LOOP type and plant group. Using these curves, it is possible, for example, to determine the frequency of a grid-related LOOP with a duration exceeding four hours. Since the frequency of all LOOPs regardless of duration can also be determined, the probability of not recovering a LOOP in a specific time period can be calculated.

The NUREG-1032 LOOP frequency curves are Weibull-based distributions. The developers of the BLACKOUT code used the values from the LOOP curves to estimate the Weibull distribution parameters for each type of LOOP. These values were listed in a table. This table is reproduced as Table B-1. 
GEM Technical Reference

Table B-1. LOOP Frequency Parameters.

\begin{tabular}{|c|c|c|c|c|}
\hline \multicolumn{2}{|c|}{ NUREG-1032 Classification } & \multirow{2}{*}{$\frac{\lambda_{0}}{0.0797}$} & \multirow{2}{*}{$\frac{\alpha}{3.3136}$} & \multirow{2}{*}{$\frac{\beta}{0.7837}$} \\
\hline Plant-Centered & I1 & & & \\
\hline & $\mathrm{I} 2$ & 0.0834 & 2.1724 & 0.8625 \\
\hline & $\mathrm{I} 3$ & 0.0854 & 1.3428 & 0.9677 \\
\hline \multirow[t]{7}{*}{ Grid-Related } & GlR1 & 0.01 & 2.9957 & 0.7108 \\
\hline & G2R1 & 0.03 & 2.8706 & 0.8053 \\
\hline & G3Rl & 0.1 & 2.5903 & 0.4704 \\
\hline & G4R1 & 0.3 & 2.8042 & 0.2879 \\
\hline & G1R2 & 0.01 & 1.4697 & 0.9899 \\
\hline & G2R2 & 0.03 & 1.1087 & 0.8665 \\
\hline & G3R2 & 0.1 & 0.9282 & 0.6865 \\
\hline \multirow{10}{*}{$\begin{array}{l}\text { Severe Weather- } \\
\text { Related }\end{array}$} & SR1R1 & 0.002 & 0.6094 & 0.1497 \\
\hline & SR2R1 & 0.005 & 1.0788 & 0.5463 \\
\hline & SR3R1 & 0.02 & 0.9039 & 0.9132 \\
\hline & SR4R1 & 0.05 & 1.2890 & 0.4821 \\
\hline & SR5R1 & 0.2 & 1.4528 & 0.3978 \\
\hline & SR1R2 & 0.002 & 1.0498 & 0.3889 \\
\hline & SR2R2 & 0.005 & 0.1985 & 0.9759 \\
\hline & SR3R2 & 0.02 & 0.2169 & 0.9910 \\
\hline & SR4R2 & 0.05 & 0.3231 & 0.8346 \\
\hline & SR5R2 & 0.2 & 0.0784 & 1.3169 \\
\hline
\end{tabular}




\begin{tabular}{lcccc}
\hline NUREG-1032 Classification & $\lambda_{0}$ & $\alpha$ & $\beta$ \\
\hline $\begin{array}{l}\text { Extremely Severe } \\
\text { Weather-Related }\end{array}$ & SS1 & 0.0002 & 0 & 0 \\
& SS2 & & & \\
& SS3 & 0.0005 & 0 & 0 \\
& SS4 & 0.002 & 0 & 0 \\
& SS5 & 0.005 & 0 & 0 \\
\hline
\end{tabular}

The Weibull distribution is in the form of

$$
\lambda(t)=\lambda_{0} e^{-\alpha t^{\beta}}
$$

where $\lambda_{0}, \alpha$, and $\beta$ are from Table B-1 for the type of LOOP under consideration, and $t$ is the LOOP duration expressed in hours.

The likelihood of failing to recover from a LOOP in time $t$ is then

$$
\lambda_{0} e^{-\alpha t^{\beta}} / \lambda_{0}=e^{-\alpha t^{\beta}}
$$

For an initiating event assessment, GEM replaces the initiating event frequency with a short-term nonrecovery probability

$$
P_{\text {LOOP }}\left(\operatorname{NoRec} \mid t_{\text {short }}\right)=e^{-\alpha t_{\text {short }}^{\beta}}
$$

where $t_{\text {short }}$ is 0.5 hour.

B-2.3.2.2 LOOP Long-Term Recoveries in BWRs. The ASP models include various AC power nonrecovery top events in the LOOP sequences. The long-term AC power nonrecovery values associated with these top events include recovery of offsite power and recovery of an emergency diesel generator. NUREG-1032 presented a median time for restoration of one diesel generator when more than one are unavailable due to independent faults as approximately four hours. The common cause failure median repair time was shown to be between two and eight hours. In the ASP models a median diesel generator repair time of four hours is used. 
The diesel generator recovery distribution density function is

$$
f_{D}(t)=\lambda_{D} e^{-\lambda_{D} t_{D G R}}
$$

where $t_{D G R}$ is the diesel generator repair time.

Thus, the cumulative diesel generator recovery distribution is

$$
F_{D}(t)=1-e^{-\lambda_{D} t_{D G R}}
$$

Solving for $\lambda_{D}$ associated with the median (50th percentile) gives

$$
\begin{gathered}
0.5=1-e^{-\lambda_{D} t_{D G R_{50}}} \\
\lambda_{D}=-\ln (0.5) / t_{D G R_{50}}=0.693 / t_{D G R_{50}}
\end{gathered}
$$

where $t_{D G R_{s}}$ is the median diesel generator repair time. The probability that at least one diesel generator is not recovered in time $t$ is

$$
P_{D G}(N o R e c)=1-F_{D}(t)=e^{-\lambda_{D} t}=e^{-0.693 t / t_{D G R_{30}}}
$$

The long-term AC power nonrecovery probability given that it was not recovered in the short term is then expressed as

$$
P_{N R A C}\left(t_{\text {long }} \mid t_{\text {short }}\right)=\left(e^{-\alpha t_{\text {long }}^{\beta}} * e^{-0.693 t_{\text {long }} / t_{D G R_{S 0}}}\right) / e^{-\alpha t_{\text {short }}^{\beta}}
$$

where $t_{\text {long }}$ is the desired recovery time under consideration, $t_{\text {short }}$ is the short-term recovery period, and $t_{D G R_{50}}$ is the median diesel generator repair time. The $\alpha$ and $\beta$ values are from Table B-1 for the type of LOOP and plant group being analyzed. GEM uses this equation directly for BWRs, with $t_{\text {long }}$ usually being the battery depletion time. The exponential term involving $t_{\text {long }}$ accounts for the offsite power 
nonrecovery and the term involving $t_{D G R_{s 0}}$ accounts for emergency diesel generator nonrecovery. The term involving $\mathrm{t}_{\text {short }}$ satisfies the condition that offsite power has not been recovered in the short-term.

B-2.3.2.3 LOOP Recoveries and Seal LOCAs in PWRs. For PWRs, the time-dependent nature of potential RCP seal LOCAs must be integrated into the calculations. The potential for a seal LOCA during station blackout is significant for many seal designs if seal cooling and seal injection are unavailable for more than about one hour.

The ASP models' LOOP event trees depict three core damage scenarios involving seal LOCA. First, a seal LOCA occurs prior to restoration of $A C$ power, $A C$ power is restored prior to core uncovery, but HPI fails to provide makeup or long-term cooling fails. Second, a seal LOCA occurs and AC power is not restored prior to core uncovery. Finally, no seal LOCA occurs but AC power is not restored prior to battery depletion. The likelihood of a seal LOCA prior to AC power recovery and the likelihood of AC power recovery are timedependent, and this time-dependency is accounted for in the analysis.

The probability of a seal LOCA at some time $t$ in some time interval dt given a blackout condition up to time $t$ is

$$
P_{S L}(t \mid N R A C)=f_{S L}(t) d t
$$

where $f_{S L}$ is the seal LOCA conditional probability density function. The seal LOCA model in ASP is based on the NUREG-1150 seal LOCA cumulative distribution function, $\mathrm{F}_{\mathrm{SL}}(\mathrm{t})$. Table $\mathrm{B}-2$ shows the seal LOCA cumulative distribution functions for the three identified seal designs. Additional options include guaranteed seal failure at 0.5 hour, one hour, or two hours, and no seal failure.

Table B-2. Seal LOCA cumulative distribution functions.

\begin{tabular}{|l|c|c|c|c|c|}
\hline \multirow{2}{*}{$\begin{array}{l}\text { Seal } \\
\text { Design }\end{array}$} & \multicolumn{5}{|c|}{ Time } \\
\cline { 2 - 6 } & $1.5 \mathrm{~h}$ & $2.5 \mathrm{~h}$ & $3.5 \mathrm{~h}$ & $4.5 \mathrm{~h}$ & $5.5 \mathrm{~h}$ \\
\hline Old O-ring & 0.70 & 0.71 & 0.73 & 0.73 & 0.73 \\
\hline New O-ring & 0.19 & 0.19 & 0.19 & 0.19 & 0.19 \\
\hline Seal Return isol. & 0.12 & 0.12 & 0.12 & 0.12 & 0.12 \\
\hline
\end{tabular}

If core uncovery occurs at time $t_{\mathrm{CU}}$ following a seal LOCA, then for a small interval dt about time $t$, the probability of a seal LOCA and unsuccessful AC power recovery within $t+t_{C U}$, given that power was unavailable at time $t$, can be written as: 


$$
P_{S L(N R A C)}(t)=P_{N R A C}\left(t \mid t_{\text {short }}\right) * f_{S L}(t) * P_{N R A C}\left(t+t_{C U} \mid t\right) d t
$$

where $P_{\text {NRAC }}\left(t \mid t_{\text {shor }}\right)$ is the probability of unsuccessful AC power recovery at time $t$ given that power was not recovered at time $t_{\text {short }}$ (a condition necessary to get a seal LOCA), $f_{S L}(t)$ is the seal LOCA density function given a blackout condition up to time $t$, and $P_{N R A C}\left(t+t_{C V} \mid t\right)$ is the probability of unsuccessful AC power recovery within the time $t+t_{C U}$ given that $A C$ power was not recovered at time $t$. Since

$$
P_{N R A C}\left(t+t_{C U} \mid t\right)=\frac{P_{N R A C}\left(t+t_{C U} \mid t_{\text {short }}\right)}{P_{N R A C}\left(t \mid t_{\text {short }}\right)}
$$

equation (15) reduces to

$$
P_{S L(N R A C)}(t)=f_{S L}(t) * P_{N R A C}\left(t+t_{C U} \mid t_{s h o r t}\right) d t
$$

To ensure that the calculation is not extended beyond the end of battery life, $t_{\text {batt }}$, since no recovery is possible once the batteries are depleted, equation (17) is modified:

$$
P_{S L(N R A C)}(t)=f_{S L}(t) * P_{N R A C}\left(\min \left(t+t_{C U}, t_{b a t t}\right) \mid t_{\text {short }}\right) d t
$$

For the situation where AC power is successfully recovered in time $t_{\mathrm{cU}}$ following the onset of a seal LOCA, the probability can be written as:

$$
P_{S L(A C R E C)}(t)=P_{N R A C}\left(t \mid t_{\text {short }}\right) * f_{S L}(t) *\left(1-P_{N R A C}\left(\min \left(t+t_{C U}, t_{b a t t}\right) \mid t\right)\right) d t
$$

where the term $P_{N R A C}\left(\min \left(t+t_{c U}, t_{b a t}\right) t\right)$ is to ensure that the calculation is not extended past the end of battery life.

By integrating equation (19) over the time period from $t_{\text {short }}$ to $t_{\text {batt }}$, we obtain the probability of a seal LOCA and successful recovery of $\mathrm{AC}$ power before core uncovery:

$$
P_{S L(A C R E C)}=\int_{t_{\text {shor }}}^{t_{\text {batt }}} P_{N R A C}\left(t \mid t_{\text {short }}\right) * f_{S L}(t) *\left(1-P_{N R A C}\left(\min \left(t+t_{C U}, t_{\text {batt }}\right) \mid t\right)\right) d t
$$


Integrating equation (18) over the same time period gives the probability of a seal LOCA and the failure to recover AC power:

$$
P_{S L(N R A C)}=\int_{t_{\text {short }}}^{t_{\text {batt }}} P_{N R A C}\left(\min \left(t+t_{C U}, t_{\text {batt }}\right) \mid t_{\text {short }}\right) f_{S L}(t) d t
$$

The probability of no seal LOCA between times $t_{\text {short }}$ and $t_{\text {batt }}$ is given as

$$
P_{N O S L}=P_{N R A C}\left(t_{\text {batt }} \mid t_{\text {short }}\right)\left(1-\int_{t_{\text {short }}}^{t_{\text {bats }}} f_{S L}(t) d t\right)
$$

Using equations (20) through (22) in various combinations generates the expressions for the seal LOCA and electric power recovery basic events in the PWR ASP models. Basic event RCS-MDP-LK-SEALS represents the probability of a seal LOCA given a station blackout condition:

$$
\begin{aligned}
R C S-M D P-L K-S E A L S & =P(\text { seal } L O C A) \\
& =\operatorname{EQN}(19)+\operatorname{EQN}(20)
\end{aligned}
$$

Basic event OEP-XHE-NOREC-BD represents the probability of not recovering AC power before battery depletion given that no seal LOCA exists:

$$
\begin{aligned}
\text { OEP-XHE-NOREC }-B D & =P(N R A C \mid \text { no seal } L O C A) \\
& =\frac{\operatorname{EQN}(22)}{(1-\operatorname{EQN}(20)-\operatorname{EQN}(21))}
\end{aligned}
$$

Basic event OEP-XHE-NOREC-SL represents the probability of not recovering AC power before core uncovery or battery depletion given a seal LOCA exists: 


$$
\begin{aligned}
\text { OEP-XHE-NOREC-SL } & =P(N R A C \mid \text { seal LOCA }) \\
& =\frac{E Q N(22)}{E Q N(20)+E Q N(21)}
\end{aligned}
$$

Other basic events representing failure to recover offsite power are used in the LOOP event tree given that some or all emergency AC power is available (at least one emergency diesel generator is operating). OEPXHE-NOREC-2H represents failure to recover offsite power given success of emergency power and success of Auxiliary Feedwater. Offsite power must be restored in time to re-establish condenser vacuum and depressurize the reactor coolant system (RCS) all before switchover to sump recirculation. It was determined that offsite power would need to be recovered within 2 hours for this set of actions to be successful. Equation (24) is used except the battery depletion time is replaced with the 2 hour value and the diesel generator median repair time is set to zero (only offsite power is being recovered). OEP-XHE-NOREC-6H represents the failure to recover offsite power given success of emergency power and failure of Auxiliary Feedwater. Offsite power must be restored prior to switchover to sump recirculation (assumed to take six hours). Again equation (24) is used, but the battery depletion time is replaced with the six hour value and the median diesel generator repair time is set to zero. 
GEM Technical Reference

\section{B-3. REFERENCES}

1. J. W. Minarick, Revised LOOP Recovery and PWR Seal LOCA Models, ORNL/NRC/LTR-89/11, August 1989.

2. P. W. Baranowsky, Evaluation of Station Blackout Accidents at Nuclear Power Plants, NUREG-1032, June 1988.

3. J. W. Minarick, et al, Precursors to Potential Severe Core Damage Accidents: 1986, A Status Report, NUREG-4674, Vol. 5, May 1988, Table 4-2. 


\section{Appendix C}

\section{Reports}





\section{Appendix C}

\section{Report}

GEM generates reports for the event ID that reflect the results pertaining to the CCDP. This appendix contains two report examples for an event assessment. In the first report, both the sequence and cut set truncation was set to $90 \%$ for the initiating event assessment. This report will include only the sequences and cut sets that contain changed events. Of these events, the contributions of the events are adding in descending order until the contributions are equal to but are not greater than $90 \%$ of the total CCDP. In the second report, the truncation values were also set to $90 \%$ for the condition assessment reports. If one attaches an additional event description as described in Step 3 of Section 3.1, GEM will place this event ID text between the Description field in the header and Basic Event Changes section of the report. 
Reports

\title{
C-1. Report Sequence and Cut Set Truncation Example - Initiating Event
}

I N I T I AT I NG EVENT ASSESSMENT

\begin{abstract}
Family :
User Name:

Event 10:

Description :

Event Name

IE-LOOP

IE-SLOCA

IE-TRAN

OEP-XHE-XE-NOREC
\end{abstract}

DRESDEN_ASP

TEST 1

Initiating Event Assessment
Model Ver :

Initiating Event :

CCDP :
IE-LOOP
$---1--1-2$

3.7E-005

BASIC EVENT CHANGES

\author{
Description \\ LOSS OF OFFSITE POWER INITIATOR \\ SMALL LOCA INITIATOR \\ TRANSIENT INITIATOR \\ OPERATOR FAILS TO RECOVER OFFSITE POWER
}

\begin{tabular}{ll} 
Base Prob & arr Prob \\
\cline { 2 - 2 } $5.9 E-006$ & $3.0 \mathrm{E}-001$ \\
$1.7 \mathrm{E}-006$ & $0.0 \mathrm{E}+000$ \\
$3.4 \mathrm{E}-004$ & $0.0 \mathrm{E}+000$ \\
$6.6 \mathrm{E}-002$ & $1.4 \mathrm{E}-003$
\end{tabular}

SEQUENCE PROBABILITIES

Truncation (\% Contribution): 90.0

$\frac{\text { Event Tree Name }}{\text { LOOP }}$

LOOP

System Name

ADS

CRDL

EPS

$\mathrm{HCI}$

OEP

P1

P2

P3

RP1
Even Tree: LOOP Current

$$
\frac{\text { CCDP }}{1.4 E-005}
$$

Even Tree: LOOP Current

$\frac{\text { CCDP }}{7.7 E-006}$

2. $0 E-006$

$1.2 E-006$
\% cut

$\frac{\text { Set }}{74.7}$

$\%$ cut

$\frac{\text { Set }}{58.3}$

15.3

9.3
Sequence Name 41

28-26

Description

AUTOMATIC DEPRESSURIZATION FAILS

INSUFFICIENT CRD FLOW TO RCS

EMERGENCY POWER SYSTEM FAILS

HPCI FAILS TO PROVIDE SUFFICIENT FLOW TO RX VESSEL

OFFSI TE POWER RECOVERY

ONE OR LESS SRV FAIL TO CLOSE

TWO SRVS FAIL TO CLOSE

THREE OR MORE SRVS FAIL TO CLOSE

REACTOR SHUTDOWN FAILS

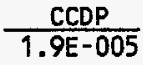

$1.3 \mathrm{E}-005$ $\frac{\% \text { Cont }}{50.2}$

35.8

\begin{tabular}{lc}
\multicolumn{2}{c}{ Logic } \\
\hline /RP1 & EPS \\
/OEP & /P3 \\
/P2 & P1 \\
HCI &
\end{tabular}

$\mathrm{HCI}$

$\begin{array}{ll}\text { /RP1 } & \text { /EPS } \\ \text { /P3 } & \text { /P2 } \\ \text { P1 } & \text { HCI } \\ \text { ADS } & \text { CRDI }\end{array}$

SEQUENCE CUT SETS

Truncation (\% Cut Set) : 90.0

Sequence: 41

CCDP: $1.9 \mathrm{E}-005$

\begin{tabular}{lll} 
& CUt Set Events \\
\hline EPS-DGN-CF-DGNS & EPS-XHE-XE-NOREC & HCI-TDP-FC-TRAIN \\
HCI-XHE-XE-NOREC & PPR-SRV-OO-TVLV & \\
& Sequence: $28-26$ & CCDP: $1.3 E-005$
\end{tabular}

\begin{tabular}{lll} 
& Cut Set Events & \\
\hline ADS-XHE-XE-ERROR & CRD-XHE-XE-NOREC & EPS-XHE-XE-NOREC \\
HCI-TDP-FC-TRAIN & HCI - XHE-XE-NOREC & PPR-SRV-DO- IVLV
\end{tabular}

EPS-DGN-FC-DG23

ADS-SRV-CC-VALVS EPS-XHE-XE-NOREC PPR-SRV-OO-IVLV

ADS-XHE-XE-NOREC HCI - TDP-FC-TRAIN EPS-DGN-FC-DG23

CRD - XHE-XE-NOREC HCI - XHE-XE-NOREC

ADS-XHE-XE-ERROR

CRD-XHE-XE-ERROR HCI -XHE-XE-NOREC

HCI - TDP-FC-TRAIN PPR-SRV-OO-1VLV 
6.0E-007

4.5

ADS-XHE-XE-ERROR

HCI - TDP-FC-TRAIN

EPS-DGN-FC-DG2

CRD - XHE-XE-NOREC

HCI - XHE - XE-NOREC

EPS-DGN-FC-DG3

BASIC EVENTS (Cut sets only)

Event Name

ADS-SRV-CC-VALVS

ADS-XHE-XE-ERROR

ADS-XHE-XE-NOREC

CRD - XHE-XE-ERROR

CRD-XHE-XE-NOREC EPS-DGN-CF-DGNS EPS-DGN-FC-DG2 EPS-DGN-FC-DG23

EPS-DGN-FC-DG3

EPS-XHE-XE-NOREC HCI - TDP - FC - TRAIN HCI - XHE-XE-NOREC PPR-SRV-OO-IVLV
Description

ADS VALVES FAIL TO OPEN

OPERATOR ERROR PREVENTS DEPRESSURIZATION

OPERATOR FAILS TO RECOVER ADS

OPERATOR FAILS TO ALIGN CRD

OPERATOR FAILS TO RECOVER CRD

COMMON CAUSE FAILURE OF DIESEL GENERATORS

UNIT 2 GENERATOR FAILS

SWING DIESEL GENERATOR FAILS

UNIT 3 DIESEL GENERATOR FAULRE

OPERATOR FAILS TO RECOVER EMERGENCY POWER

HPCI TRAIN LEVEL FAILURES

OPERATOR FAILS TO RECOVER HPCI

ONE OR LESS SRVS FAIL TO CLOSE
EPS - XHE-XE-NOREC

PPR-SRV-DO-IVLV

\begin{tabular}{c} 
Curr Prob \\
\hline $3.7 \mathrm{E}-003$ \\
$1.0 \mathrm{E}-002$ \\
$7.1 \mathrm{E}-001$ \\
$1.0 \mathrm{E}-002$ \\
$1.0 \mathrm{E}+000$ \\
$1.4 \mathrm{E}-003$ \\
$7.8 \mathrm{E}-002$ \\
$7.8 \mathrm{E}-002$ \\
$7.8 \mathrm{E}-002$ \\
$8.0 \mathrm{E}-001$ \\
$1.6 \mathrm{E}-001$ \\
$7.1 \mathrm{E}-001$ \\
$3.6 \mathrm{E}-001$
\end{tabular} 
Reports

\section{C-2. Report Sequence and Cut Set Truncation Example - Condition Assessment}

CONDITION ASSESSMENT

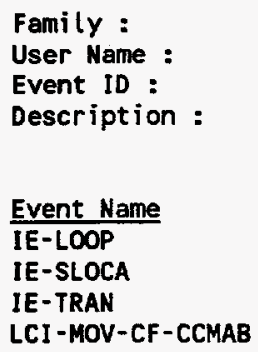

Event Name

IE-LOOP

IE-SLOCA

IE-TRAN

LCI - MOV-CF-CCMAB

BASIC EVENT CHANGES

Event Tree Name
Description

LOSS OF OFFSITE POWER INITIATOR

SMALL LOCA INITIATOR

TRANSIENT INITIATOR

COMMON CAUSE FAILURE OF THE LPCI INJECT VALVES
SEQUENCE PROBABILITIES

Truncation ( $\%$ Contribution) :
09

Sequence Name

05

$37-04$

04

\begin{abstract}
Difference
6.1E-008
\end{abstract}

$2.2 \mathrm{E}-008$

$1.6 E-008$

9.5E-009
Model Ver :

Duration (hours) : CCDP :

$-1-1--1-1$

$2.2 E+001$

1.2E-007
TRAN

LOOP

TRAN

System Name
CCM
CCML
CVS
EPS
HCI
MFW
P1
P2
P3
PCS
RP1
RPS
SDC
SDCL

90.0

$\frac{\% \operatorname{con} t}{50.3}$

/RPS
/P3
/P1
SDC
CVS

18.1 /RPS

IP3

P1

SDC

$13.5 \stackrel{\text { /RP1 }}{/ P 3}$

/P3

SOCL.

CVS

7.8 /RPS

IP3

/HCI

CCM

$\begin{array}{ll}\text { Base Prob } & \text { arr Prob } \\ 5.9 E-006 & 1.3 E-004 \\ 1.7 E-006 & 3.7 E-005 \\ 3.4 E-004 & 7.5 E-003 \\ 2.6 E-004 & 1.0 E+000\end{array}$

Description

CONTAINMENT COOLING MOOE OF LPCI

CONTAINMENT COOLING MODE OF LPCI

CONTAINMENT (SUPPRESSION POOL) VENTING

EMERGENCY POWER SYSTEM FAILS

HPCI FAILS TO PROVIDE SUFFICIENT FLOW TO RX VESSEL

FEEDWATER SYSTEM FAILS

ONE OR LESS SRV FAIL TO CLOSE

TWO SRVS FAIL TO CLOSE

THREE OR MORE SRVS FAIL TO CLOSE

POWER CONVERSION SYSTEM

REACTOR SHUTDOWN FAILS

REACTOR SHUTDOWN FAILS

SHUTDOWN COOLING SYSTEM FAILS

SHUTDOWN COOLING SYSTEM FAILS 
SEQUENCE CUT SETS

Truncation (\% Cut Set) : 90.0

Even Tree: TRAN

$\begin{array}{ll}\begin{array}{c}\text { Current } \\ \text { CCDP }\end{array} & \begin{array}{c}\text { \% Cut } \\ \text { Set }\end{array} \\ 1.4 E-008 & 23.7 \\ 1.4 E-008 & 23.7 \\ 1.4 E-008 & 23.7 \\ 8.8 E-009 & 14.5\end{array}$

Even Tree: TRAN

Current

$\frac{\text { CCDP }}{5.2 \mathrm{E}-009}$

\begin{tabular}{l}
$\%$ Cut \\
Set \\
\hline
\end{tabular}

23.7

5.2E-009

23.7

5.2E-009

3.2E-009

14.5

Even Tree: LOOP

Current

$\frac{\text { CCDP }}{9.4 E-009}$

$\%$ Cut

$\frac{\text { Set }}{57.4}$

1.5E-009

9.0

1.5E-009

9.0

$1.5 E-009$

9.0
Sequence: 05

Cut Set Events

LCI -XHE-XE-NOREC

SDC-MDP-FC-TRNA

LCI -XHE-XE-NOREC SDC-MDP-FC-TRNB

LCI - XHE-XE-NOREC SDC-MDP-FC-TRNC

E-XE-VENT PCS-XHE-XE-NOREC

LCI -XHE-XE-NOREC SDC-MOV-FC-INJEC

CVS-XHE-XE-VENT
PCS-XHE-XE-NOREC

Sequence: 37-04

Cut Set Event

LCI -XHE-XE-NOREC

PPR-SRV-00-1VLV

CCDP:

$6.1 E-008$

CVS-XHE-XE-VENT

PCS-XHE-XE-NOREC

SOC-XHE-XE-NOREC

CVS-XHE-XE-VENT

PCS-XHE-XE-NOREC

SDC-XHE-XE-NOREC

CVS-XHE-XE-VENT

PCS-XHE-XE-NOREC

SDC-XHE-XE-NOREC

CVS-XHE-XE-VENT

PCS-XHE-XE-NOREC

SDC-XHE-XE-NOREC

Sequence: 04

LCI -XHE-XE-NOREC

PPR-SRV-0O-1VLV

LCI -XHE-XE - NOREC

PPR-SRV-0O-1VLV

LCI -XHE-XE-NOREC

PPR-SRV-00-1VLV

SDC-MOV-FC-INJEC

CCDP: $1.6 E-008$

Cut Set Events

CVS-XHE-XE-VENT

SDC-XHE-XE-NOREC

EPS-XHE-XE-NOREC

EPS-DGN-FC-DG23

CVS-XHE-XE-VENT

SDC-XHE-XE-NOREC

LCI -XHE-XE-NOREC

LCI -XHE-XE-NOREC

SDC-XHE-XE-NOREC

LCI-XHE-XE-NOREC

SDC-MDP-FC-TRNA

LCI -XHE-XE-NOREC

SOC-MDP-FC-TRNC

SDC-MDP-FC-TRNB

SDC-XHE-XE-NOREC 
Reports

Even Tree: TRAN

Current

$\frac{\text { CCDP }}{2.2 E-009}$

2.2E-009

2. $2 E-009$

1.4E-009

14.5

23.7

\begin{tabular}{lll} 
& CUt Set EVents & \\
\hline CVS-XHE-XE-VENT & LCI-XHE-XE-NOREC & MFW-SYS-VF-FEEDN \\
MFW-XHE-XE-NOREC & PCS-SYS-VF-MISC & PCS-XHE-XE-NOREC \\
SDC-MDP-FC-TRNA & SDC-XHE-XE-NOREC &
\end{tabular}

CVS-XHE-XE-VENT MFW-XHE-XE-NOREC SDC-MDP-FC-TRNB

CVS-XHE-XE-VENT MFW-XHE-XE-NOREC SDC-MDP-FC-TRNC SDC-XHE-XE-NOREC

LCI - XHE-XE-NOREC PCS-SYS-VF-MISC SDC-XHE-XE-NOREC

LCI - XHE-XE-NOREC PCS-SYS-VF-MISC SDC-XHE-XE-NOREC

CVS-XHE-XE-VENT MFW-XHE-XE-NOREC SDC-MOV-FC-INJEC

LCI - XHE-XE-NOREC PCS-SYS-VF-MISC SDC-XHE-XE-NOREC

BASIC EVENTS (Cut Sets Only)

Event Name

CVS-XHE-XE-VENT EPS-DGN-FC-DG23 EPS-XHE-XE-NOREC LCI -XHE-XE-NOREC MFW-SYS-VF-FEEDW MFW-XHE-XE-NOREC PCS-SYS-VF-MISC PCS-XHE-XE-NOREC PPR-SRV-OO-IVLV SDC-MDP-FC-TRNA SDC-MDP-FC-TRNB SDC-MDP-FC-TRNC SDC-MOV-FC-INJEC SDC-XHE-XE-NOREC

Description

OPERATOR FAILS TO VENT CONTAINMENT

SWING DIESEL GENERATOR FAILS

OPERATOR FAILS TO RECOVER EMERGENCY POWER

OPERATOR FAILS TO RECOVER LPCI

FEEDWATER HARDWARE COMPONENTS FAIL

OPERATORS FAIL TO RECOVER FEEDWATER

PCS HARDWARE COMPONENTS FAIL

OPERATOR FAILS TO RECOVER PCS

ONE OR LESS SRVS FAIL TO CLOSE

SDC TRAIN A COMPONENTS FAIL

SDC TRAIN B COMPONENTS FAIL

SDC TRAIN C COMPONENTS FAIL

SDC INJECTION PATH FAILS

OPERATOR FAILS TO RECOVER SDC
MFW-SYS-VF-FEEDW

PCS-XHE-XE-NOREC

MFW-SYS-VF-FEEDIN

PCS-XHE-XE-NOREC

MFW-SYS-VF-FEEDW PCS-XHE-XE-NOREC 


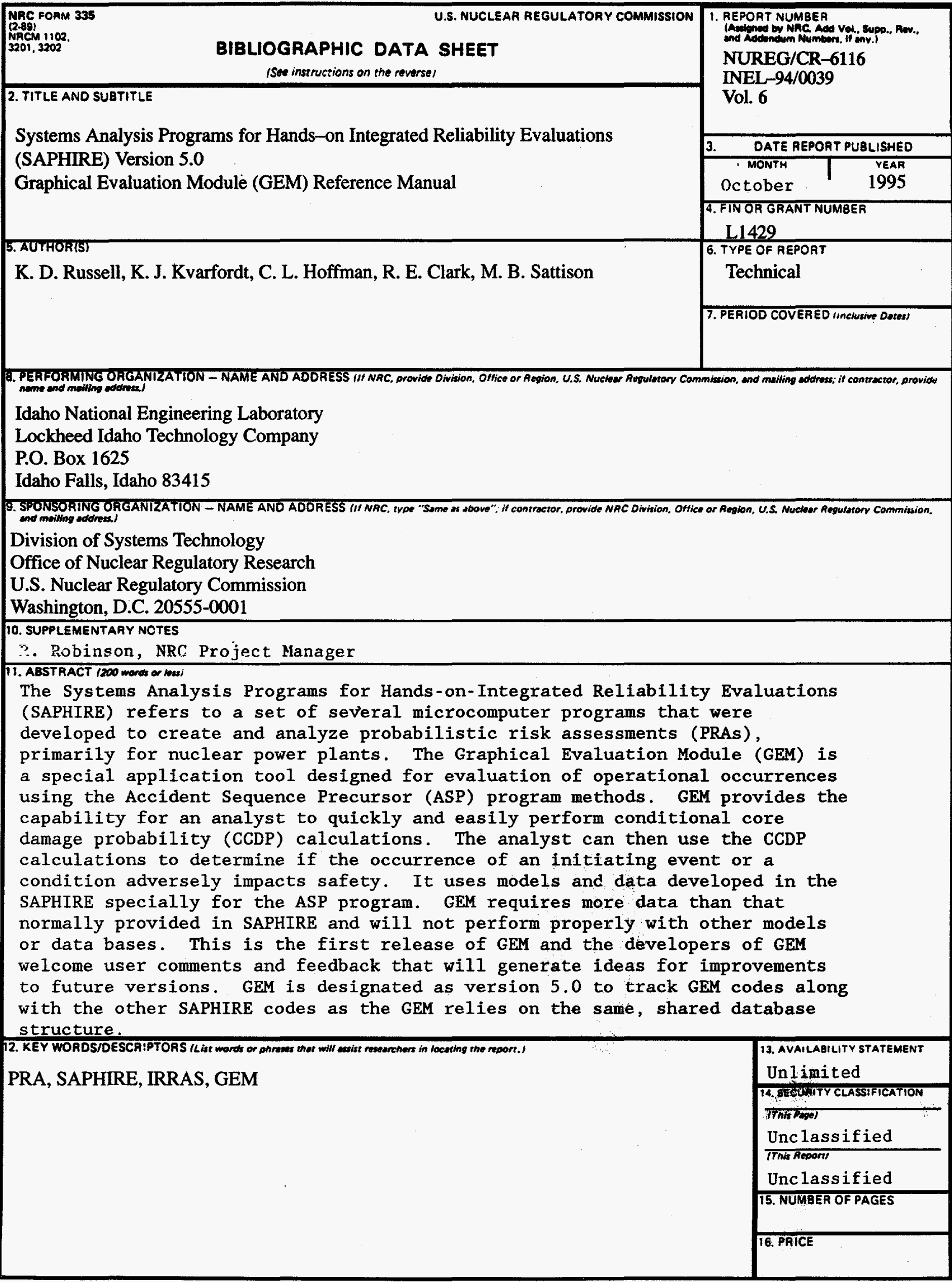

